\title{
DOE/CH/OSOOO-TM
}

\section{Do Central Tracking Chamber Performance Studies}

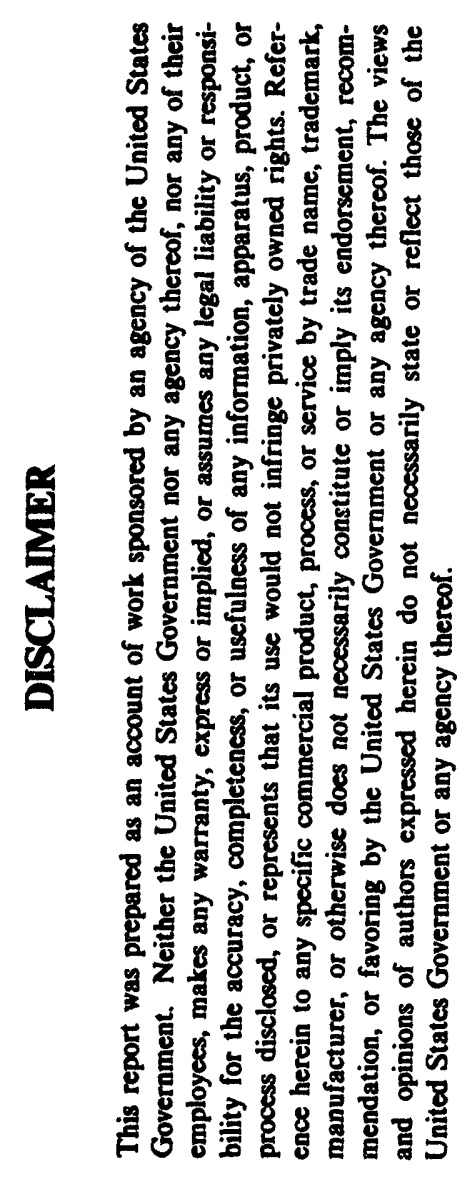

\author{
A Dissertation Presented \\ by \\ Domenico Pizzuto \\ to \\ The Graduate School \\ in Partial Fulfillment of the Requirements \\ for the Degree of \\ Doctor of Philosophy \\ in \\ Physics \\ State University of New York \\ at \\ Stony Brook
}

December 1991

ACO2-76CHO300O 


\section{State University of New York \\ at Stony Brook \\ The Graduate School \\ Domenico Pizzuto}

We, the dissertation committee for the above candidate for the Doctor of Philosophy degree, hereby recommend acceptance of the dissertation.
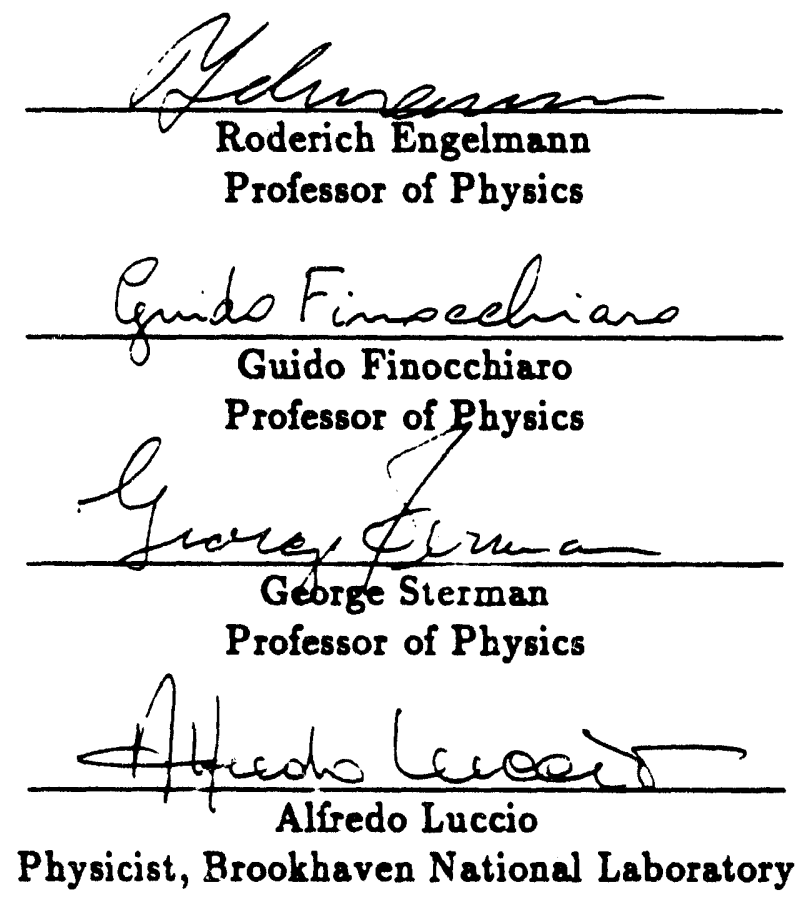

This dissertation is accepted by the Graduate School.

Graduate School 


\title{
Abstract of the Dissertation Do Central Tracking Chamber Performance Studies
}

\author{
by \\ Domenico Pizzuto \\ Doctor of Philosophy \\ in \\ Physics \\ State University of New York at Stony Brook \\ 1991
}

The performance of the completed Do central tracking chamber was studied using cosmic rays at the State University of New York at Stony Brook. Also studied was a prototype tracking chamber identical in design to the completed Do tracking chamber. The prototype chamber was exposed to a collimated beam of $150 \mathrm{GeV}$ pions at the Fermilab NWA test facility. Results indicate an $R \Phi$ tracking resolution compatible with the limitations imposed by physical considerations, excellent 2 track resolution, and a high track reconstruction efficiency along with a good rejection power against $\gamma \rightarrow e^{+} e^{-}$events. 
To my mother and father

1

Nom 


\section{Contents}

List of Figures $\ldots \ldots \ldots \ldots \ldots \ldots \ldots \ldots$ viii

List of Tables $\ldots \ldots \ldots \ldots \ldots \ldots \ldots \ldots \ldots$ xiii

Acknowledgements $\ldots \ldots \ldots \ldots \ldots \ldots \ldots$ xiv

1 The Do Detector $\ldots \ldots \ldots \ldots \ldots \ldots \ldots \ldots$

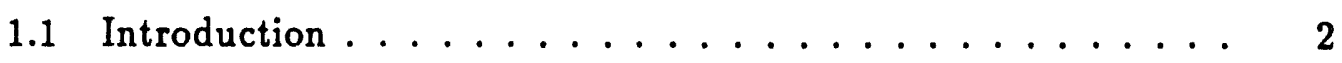

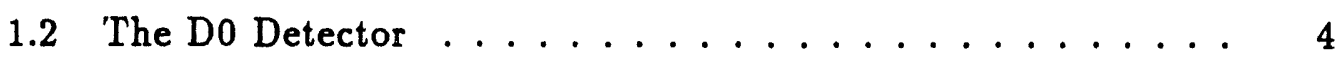

1.3 The D0 Central Detector System $\ldots \ldots \ldots \ldots \ldots$

1.3.1 The Do Vertex Chamber . . . . . . . . . . . . 5

1.3.2 The Do Transition Radiation Detector . . . . . . . 7

1.3.3 The Do Forward-Backward Drift Chambers . . . . 8

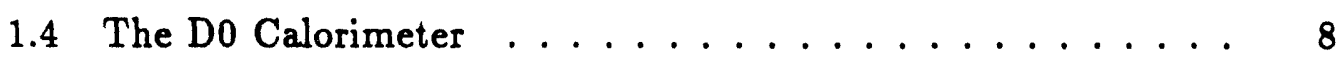

1.5 The Muon Detector . . . . . . . . . . . . . 12

2 The Central Drift Chamber Mechanics and Signal Processing 14

2.1 The Stony Brook Central Tracking Chamber . . . . . . . . 15

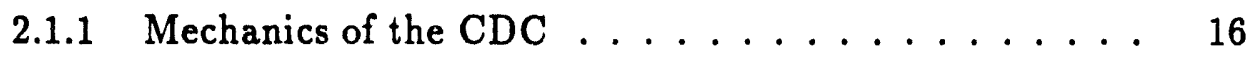

2.1.2 Field Shaping Within the Drift Cell . . . . . . . . 17

2.2 Signal Processing and Data Acquisition . . . . . . . . 20

2.2 .1 Electronics Overview . . . . . . . . . . . . 20

2.2.2 Hardware Zero Suppression $\ldots \ldots \ldots \ldots \ldots$ 
2.2.3 Electronic Calibration . . . . . . . . . . . . 24

2.3 Software Hitfinding . . . . . . . . . . . . . . . . 24

3 Theory of Electron Drift in Gases . . . . . . . . . . 28

3.1 Transport Theory of Electron Drift in Gases . . . . . . . 29

3.1.1 Electron Energy Distribution and Drift Velocity . . . . 29

3.1.2 Elastic Collisions . . . . . . . . . . . . . . 30

3.2 Application to Polyatomic and Multi Component Gas Mixtures 32

3.2.1 Polyatomic Gases (Inelastic Collisions) . . . . . . . 34

3.2.2 Electron Diffusion . . . . . . . . . . . . . . . . 35

3.2.3 Multicomponent Gases . . . . . . . . . . . . 35

$4 R \Phi$ Tracking $\ldots \ldots \ldots \ldots \ldots \ldots \ldots \ldots \ldots \ldots$

4.1 Segment Finding . . . . . . . . . . . . . 38

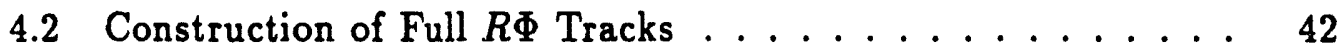

5 CDC Performance Studies $\ldots \ldots \ldots \ldots \ldots \ldots \ldots$

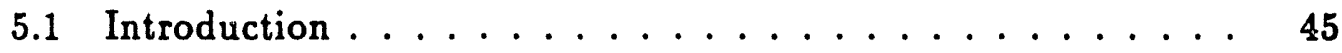

5.2 Cosmic Ray Experimental Setup . . . . . . . . . . . . . . 45

5.3 Fermilab Test Beam Experimental Setup . . . . . . . . . . 46

5.4 Electronic Delays . . . . . . . . . . . . . . . . . 47

5.4 .1 Cosmic data . . . . . . . . . . . . 47

5.4 .2 Fermilab Data . . . . . . . . . . . . . 48

5.5 Drift Velocity . . . . . . . . . . . . . . 52

5.5 .1 Fermilab Data . . . . . . . . . . . . . . . 52

5.5.2 Cosmic Ray Data . . . . . . . . . . . . . . 62

5.6 Sense Wire Efficiencies . . . . . . . . . . . . . . 66

5.7 Sense Wire Resolution . . . . . . . . . . . . . . 69

5.7.1 Near Anode Region . . . . . . . . . . . . . 72

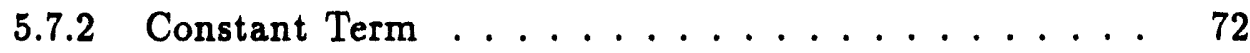


5.7.3 Diffusion Term . . . . . . . . . . . . 77

5.7.4 Effect of Angled Segments and Nonlinearities . . . . 77

5.8 Two Track Resolution in $R \Phi \ldots \ldots \ldots \ldots \ldots \ldots \ldots$

$5.9 d E / d X \ldots \ldots \ldots \ldots \ldots \ldots \ldots \ldots \ldots$

5.10 Drift Velocity Studies . . . . . . . . . . . . . . 85

5.10 .1 Mean Velocity and Velocity Step . . . . . . . . 85

5.10.2 Pressure and Temperature Dependence . . . . . . . 87

5.10.3 Gas Mix and E Field Dependence . . . . . . . . 89

5.10 .4 Nonlinearities . . . . . . . . . . . . . . . 89

5.11 Tracking Across 4 Layers . . . . . . . . . . . . . 95

5.11 .1 Alignment Procedure . . . . . . . . . . . . 95

$5.11 .2 R \Phi$ Tracking Resolution . . . . . . . . . . . 97

5.12 Monte Carlo Study of Alignment Strategies . . . . . . . . . 97

5.13 Measuring Z With Delay Lines . . . . . . . . . . . . 102

5.14 Delay Line Calibration . . . . . . . . . . . . . . . 101

5.15 Delay Line Resolution and Efficiency . . . . . . . . . . 106

6 The Search For Top Quark Production at Fermilab . . . 108

6.1 Introduction . . . . . . . . . . . . . . . . . . . . 109

6.2 Top Production and Signal For $M_{t}>M_{W} \ldots \ldots \ldots \ldots$

6.2.1 Top Search in Dilepton Channel . . . . . . . . . . . 111

6.2.2 Top Search in Lepton + Jets Channel . . . . . . . . 113

6.3 Conclusion . . . . . . . . . . . . . . . 115 


\section{List of Figures}

1.1 Configuration of the Do central detectors. . . . . . . . 5

1.2 Vertex chamber resolution in the azimuthal plane. . . . . . 6

1.3 Transition radiation detector pion rejection factor versus electron identification efficiency. ............. 7

1.4 Cross section of the Do calorimeter . . . . . . . . . 10

1.5 Cross section of a calorimeter module showing the arrangment of absorber, liquid argon gaps, and signal boards. . . . . . 11

1.6 Bend plane cross section of the D0 muon detector. . . . . . . 13

2.1 $R \Phi$ cross section of the Stony Brook central tracking chamber. 15

2.2 RZ cross-section of the completed Stony Brook central tracking chamber. ................... 16

2.3 A single module as seen from a cutaway of the end plate. . . . 17

$2.4 R \Phi$ crossection showing major components of the drift cell. . . 18

2.5 Schematic of the field shaping elements of the drift cell. . . . . 19

2.6 Electric field configuration within a drift cell. . . . . . . . . 20

2.7 Layout of signal processing chain. . . . . . . . . . 21

2.8 Signal processing stages of FADC card. . . . . . . . . . 22

2.9 Hardware zero suppression of raw FADC data. . . . . . . . 27

4.1 Two possible coordinates of a sense wire hit within a single drift

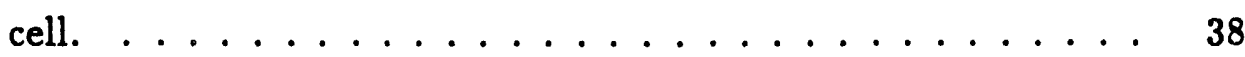

viii 
4.2 Association of sense wire hits within a segment candidate in the $R \Phi$ plane (not to scale). . . . . . . . . . . . . 40

4.3 Parameterization of segment fit. . . . . . . . . . . . 41

4.4 Association of two segments as part of a full $R \Phi$ track. . . . 42

5.1 Fermilab premod test experimental setup. . . . . . . . . 46

5.2 Leading edge of electron arrival time distribution. . . . . . . 48

5.3 Scatter plot of drift time versus PWC drift coordinate before correction for overall T0 and sense wire position. . . . . . . .

5.4 Drift time vs. drift distance for a single sense wire before position and overall To correction. . . . . . . . . . . . 51

5.5 Drift time vs. drift distance for a single sense wire after position and overall To correction. . . . . . . . . . . . . 51

5.6 Mean drift velocitites as calculated from the Fermilab test data. 53

5.7 $R \Phi$ crossection of part of the central drift chamber showing the relationship between drift cells and half modules. . . . . . . 54

5.8 Residual vs drift distance functions for sense wires in layer 0. . 55

5.9 Residual vs drift distance functions for sense wires in layer 1. . 56

5.10 Residual vs drift distance functions for sense wires in layer 2. . 57

5.11 Residual vs drift distance functions for sense wires in layer 3. . 58

5.12 Averaged residual vs drift distance functions. . . . . . . . 59

5.13 The four residual vs drift distance shapes (correction functions) which characterize the central tracking chamber. The function coefficients are summarized in table . . . . . . . . . 60

5.14 A half module of the central tracking chamber in a coordinate system in which the $X$ axis is aligned along the layer 0 - layer 2 sense wire plane. . . . . . . . . . . . .

5.15 Drift velocity and velocity step distribution for a typical $1 / 2$ module of the CDC. . . . . . . . . . . . . . 
5.16 Inner sense wire efficiencies. . . . . . . . . . . 67

5.17 Outer sense wire efficiencies. . . . . . . . . . . 68

5.18 Inner and outer sense wire resolution vs. drift distance for the CDC drift cell. ................... 71

5.19 Mean sense wire pulse shapes at various drift distances. . . . . 73

5.20 Calculated drift time vs ionization position along the sense wire gap for $3 \mathrm{~cm}$ of drift. . . . . . . . . . . . . 75

5.21 Mean arrival time distribution for inner and outer sense wires at $3 \mathrm{~cm}$ of drift showing effects of dispersion. . . . . . . . 76

5.22 Measured pulse arrival time versus displacement. . . . . . . 76

5.23 Decrease in resolution for track segments making an angle $|\Phi|$ with respect to the sense wire plane. . . . . . . . 78

5.24 Two hit resolution efficiency versus pulse seperation. . . . . 80

5.25 Single and double track mean sense wire charge distributions. Each entry in the histogram is an average of the lowest $70 \%$ of the collected sense wire charges. . . . . . . . . . . 83

5.26 Two particle rejection power vs truncated mean. . . . . . . . 83

5.27 Mean sense wire charge vs drift distance for inner and outer wires. 84

5.28 (a) Normalized mean charge on sense wires for single track events vs. theta of full track. The data points are normalized with respect to the mean charge at $\Theta=1.57$ radians. (b) Scaled harmonic mean normalized charge distribution for a typical sense wire. . . . . . . . . . . . . . . 84

5.29 Mean velocity of inner and outer sense wires for all 64 half modules of the complete central tracking chamber. . . . . . .

5.30 Mean velocity step of inner and outer sense wires for all 64 half modules of the complete central tracking chamber. . . . . . 86 
$5.31 \frac{P}{V} \frac{d V}{d P}$ vs. $P$ and $\frac{T}{V} \frac{d V}{d T}$ vs. $T$ for Mark 2 gas at $E=640 \mathrm{~V} / \mathrm{cm}$ (theory)....................... 88

5.32 Measured normalized mean drift velocity of a single half module and normalized atmospheric pressure versus time (arb. units). $\quad 88$

5.33 Theoretical electron drift velocity vs. electric field strength . . 90

5.34 Mean residual versus drift distance of outer sense wire hits with respect to a track segment formed from the inner sense wires. Results are before applying drift distance dependent correction

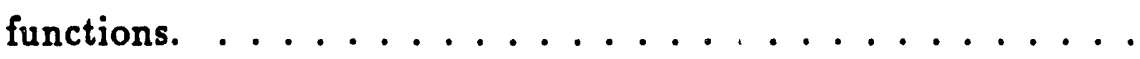

5.35 Mean residual versus drift distance of outer sense wire hits with respect to a track segment formed from the inner sense wires before and after applying drift distance dependent correction functions. ....................

5.36 Mean residual versus drift distance of layer 0 and layer 2 segment with respect to a 2 segment track formed from layers 1 and 3. Results are before applying drift distance dependent correction functions. . . . . . . . . . . .

5.37 Mean residual versus drift distance of layer 0 and layer 2 segment with respect to a 2 segment track formed from layers 1 and 3 beíore and after applying drift distance dependent correction

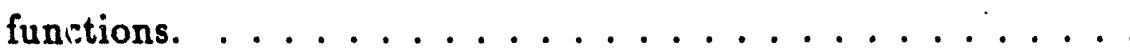

5.38 Mear: residuals and resolution with respect to a 4 layer track fit of the inner sense wires before and after corrections. . . . . . 98

5.39 Comparison of alignment strategies showing difference between reconstructed and real Monte Carlo track at TRD and EMC boundaries for various values of initial parameters. . . . . . . 101

5.40 Simplified schematic of delay line operation. . . . . . . . 102

5.41 Simplified schematic of delay line calibration system. . . . . 105 
5.42 Determination of delay line velocity and sense wire side time offset. .......................... 105

5.43 Fitted delay line mean velocities and percent rms deviation from the mean for all 256 delay lines. . . . . . . . . . . . . . . 106

5.44 Delay line resolution with respect to a full $R Z$ track fit and efficiency. Results are for all 256 delay lines. . . . . . . . . . 107

6.1 Feynman graphs contributing to the QCD creation of $t \bar{t}$. . . 110

6.2 Decay modes of the top quark and the $W\left(M_{t}>M_{W}\right)$. . 110

6.3 Invariant mass distributions of best $W$-jet pair for 3 different top quark masses. . . . . . . . . . . . . . . 116 


\section{List of Tables}

¿.1 Energy and Position Resolution of the DO Calorimeter . . . 12

5.1 Coefficients of residual correction functions . . . . . . . . 61

5.2 Summary of sense wire efficiencies . . . . . . . . . . 66

5.3 Sense wire resolution vs. drift distance function coefficients . . 70

6.1 Top decay channels and branching ratios . . . . . . . . . 111

$6.2 . t \bar{t}$ and $Z^{0} \rightarrow \tau^{+} \tau^{-}$rate for various cuts . . . . . . . 113 


\section{Acknowledgements}

I would like to thank my mother and father for their unwavering support and guidance. They have always been unselfish and caring even when I sometimes failed to do the same. To Marina and Jacqueline - I Love You. Thanks for laughing with me when I was happy and consoling me when the pressures of graduate school seemed too much to bear.

To Professor Roderich Engelmann I extend a heartfelt embrace for acting not only as my advisor but also as my motivator, psychiatrist, and most important of all - friend.

Many thanks to Professor Guido Finocchiaro and to Ties Behnke for the design and construction of the D0 Central tracking chamber. Their intelligence and hard work made the writing of this dissertation possible.

To my dear colleagues Vito Manzella, Jack Steffens, Joan Napolitano, Alice Dugan, Jim Cochran, Joey Thompson, and the High Energy Physics faculty - thanks for giving me the advice and support necessary to complete my work. 


\section{Chapter 1}

\section{The Do Detector}




\subsection{Introduction}

The DO detector is currently near completion at the Fermi National Accelerator Laboratory in Batavia, Illinois. It is the second major proton antiproton detector at Fermilab designed to study the elementary structure of matter and its interactions. With a center of mass energy of nearly $2 \mathrm{TeV}$, the Fermilab Tevatron is the most powerful hadron collider currently in existence. The upcoming collider run in March of 1992 is expected to yield a rich harvest of interesting physics, and possibly, unexpected phenomena not currently explained by existing models of nature.

We currently believe that the world is made up of a small number of elementary constituents. The electron $(e)$, muon $(\mu), \operatorname{tau}(\tau)$ and their associated neutrinos $(\nu)$ are collectively known as leptons. Particles such as the proton and neutron, once thought to be elementary, are now known to be composite objects consisting of quarks $(q)$ and the "glue" which holds them together the gluons $(g)$. The seemingly different aspects of electricity and magnetism have been unified and are now precisely understood in terms of the carrier of the electromagnetic force - the photon $(\gamma)$. Until recently, it was believed that the weak force responsible for particle decay was a seperate force completely unrelated to the other known forces of nature. We now understand the laws governing electromagnetic and weak interactions as arising naturally by imposing simple symmetry principles on the equation of motion of a noninteracting (free) particle. The resulting electroweak theory known as the Standard Model, and its three weak force mediating gauge bosons - the $W^{+}, W^{-}$, and $Z^{\circ}$, have been spectacularly confirmed by experiment. It is with regard to the observation of these elementary particles $\left(e, \mu, \nu, q, g, \gamma, W^{+}, W^{-}, Z^{\circ}\right)$ that the Do detector was designed.

In general, the identification of the elementary particles is accomplished through the analysis of signals generated by several different types of detec- 
tors. Of particular importance is the ability to accurately track the trajectory of charged particles originating from the primary interaction vertex. The absence of a central magnetic field in the DO detector design prevents particle identification by the central tracking chamber alone (discussed in the main body of this dissertation), however, the existence or non existence of a track pointing into other detector components is often the key to an unambiguous lepton identification. With the exception of neutrinos, leptons are charged particles and ,therefore, will be observed in the central tracking chamber. A distinctive signature of the gauge bosons $W^{+}, W^{-}$and $Z^{\circ}$ is their decay into an energetic charged lepton(s). Here again, a tracking chamber is vital for observing the lepton(s). Conversely, the absence of a track in conjuction with other detector activity is often the signature for a photon. Finally, heavy quark production i.e $p \bar{p} \rightarrow t \bar{t}$ (see chapter 6), is inferred through the observation of energetic charged leptons resulting from the heavy quark decay. In summary, the likelyhood of final state charged leptons resulting form elementary particle interactions demands a well calibrated, high precision tracking chamber. A general detailed discussion of the relevant physics issues to be explored at Fermilab in the 1990's can be found in several sources. ${ }^{1,2}$ 


\subsection{The Do Detector}

The D0 detector is designed to optimize the detection of what is currently believed to be the relevant fundamental particles of nature. Quarks and gluons manifest themselves as a collimated spray of hadrons (jets) originating from the primary interaction point. For this, it is necessary to design a calorimeter with a good energy resolution and a fine enough segmentation so that a precise determination of the jet energy and direction can be established. The presence of a neutrino is inferred by the observation of an energy imbalance or net energy flow within the calorimeter. Such a measurement mandates a hermetic calorimeter, that is, a calorimeter with few dead areas or cracks from which energy can escape. Electron identification requires the cooperative union of several types of detectors. Tracking chambers near the beam pipe and before the calorimeter, in conjunction with a transition radiation detector, are necessary to discriminate primary electron showers in the calorimeter from those produced by photons and $\pi^{\circ} \mathrm{s}$. Muons are observed based on their ability to traverse large quantities of dense material while maintaining a large fraction of their initial energy. Their momentum is measured by deflection in magnetized steel toroids. The ability of the DO detector to measure the energy and momentum of leptons is of fundamental importance in studying the properties of the weak vector bosons $\left(W^{+}, W^{-}\right.$and $\left.Z^{\circ}\right)$.

An overview of each of these DO detector components with the exception of the central tracking chamber is presented in the following sections. A detailed account of the mechanics and performance characteristics of the Do central tracking chamber is presented in the main body of this dissertation. 


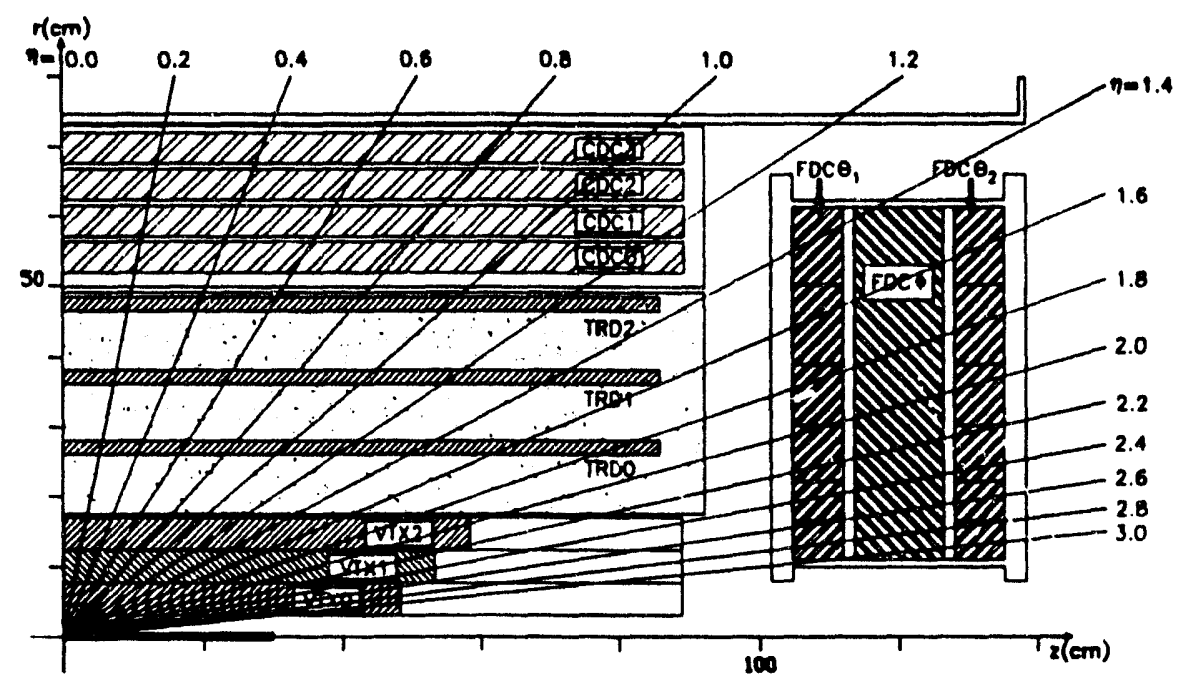

Figure 1.1: Configuration of the Do central detectors.

\subsection{The Do Central Detector System}

The central detectors of the DO experiment (figure 1.1) consist of a high resolution vertex tracking chamber (VTX) immediately surrounding the beam pipe, a transition radiation detector (TRD) for electron identification, and finally, a central (CDC) and forward-backward (FDC) set of tracking drift chambers for precision charged particle tracking in the region between the TRD and the calorimeter. The following sections give an overview of the different central detectors. The central tracking chamber mechanics and performance is discussed in detail in subsequent chapters.

\subsubsection{The Do Vertex Chamber}

The primary functions of the vertex chamber include a precise determination of the interaction vertex and secondary vertices arising from particle 


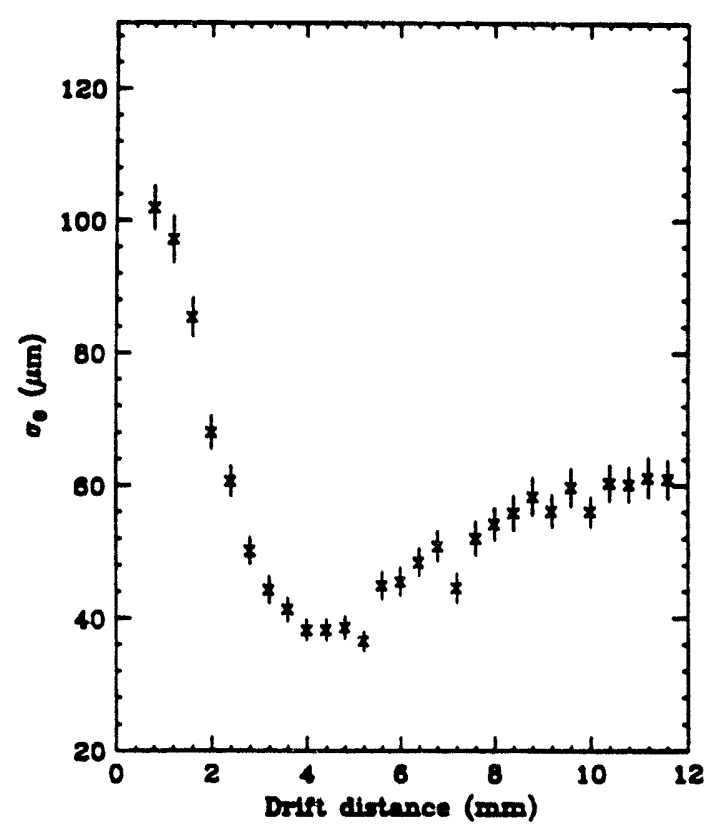

Figure 1.2: Vertex chamber resolution in the azimuthal plane.

decays near the beam pipe, and rejection of photons which convert into $e^{+} e^{-}$ pairs after the VTX. Structurally, the VTX ${ }^{3}$ consists of three mechanically independant layers with each layer azimuthally subdivided into 16, 32, and 32 seperate drift cells repectively. Each drift cell is defined by a multiwire electric field shaping cage (cathode). At the center of each cage are 8 sense wires running parallel to the beam line and radially seperated by $4.6 \mathrm{~mm}$. The sense wires serve as the anodes of the field cage. The coordinates of a track in the azimuth are given by sense wire induced pulses arising from the drift of ionized electrons produced by a charged particle traversing the VTX. The axial coordinate (along the beam pipe) is given by measuring the ratio of collected charge on both sides of the sense wires (charge division).

The need for a high spatial resolution and resolving power for closely spaced tracks demand a low diffusion, low drift velocity gas. $\mathrm{A} \mathrm{CO}_{2}(95 \%)$ ethane $(5 \%)$ mixture was chosen to fulfill these requirements. Current tests of 


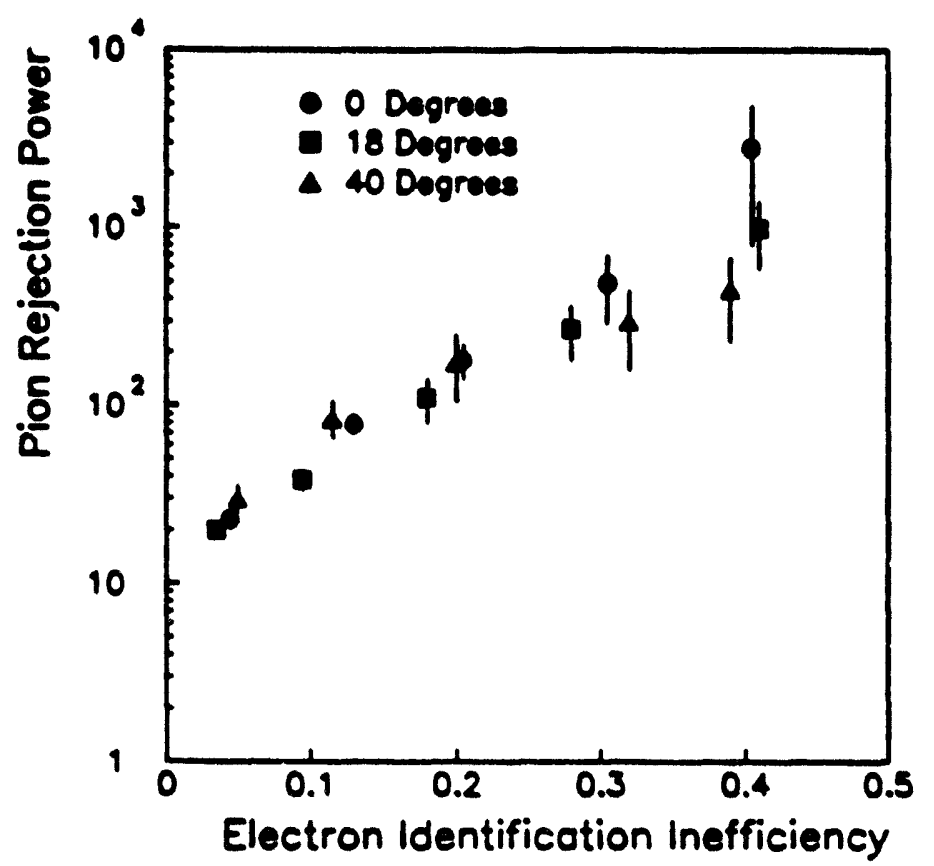

Figure 1.3: Transition radiation detector pion rejection factor versus electron identification efficiency.

the VTX at Fermilab have shown a spatial resolution of $60 \mu \mathrm{m}$ or better (figure 1.2) for drift distances larger than $2 \mathrm{~mm}$ and a resolving efficiency of $90 \%$ for a two track seperation of $630 \mu m$.

\subsubsection{The Do Transition Radiation Detector}

Transition radiation is produced when a charged particle crosses the boundary between materials with different dielectric constants. In general, the field configuration surrounding a charged particle will be modified when inside a dielectric material due to the presence of an additional polarization field arising in response to the particle's electric field. This reorganization of the electric field at the interface between materials with different dielectric properties results in photon emission ( $\mathrm{X}$-rays) known as transition radiation. The transition radiation becomes appreciable only if the particle is highly relativistic 
$(\gamma \approx 500)$ with the total emitted energy being proportional to $\gamma{ }^{4}$ It is precisely this $\gamma$ dependance on the emitted energy which makes transition radiation attractive for electron identification.

The DO transition radiation detector ${ }^{5}$ consists of three concentric stacks of $18 \mu \mathrm{m}$ thick polypropylene foils used as the dielectric medium. Each stack contains 400 foils seperated by $150 \mu m$ gaps. Since the probability of energetic photon emission within a single thin foil is low, a series of many foils must be used to obtain an appreciable photon yield. The $\mathrm{X}$-ray photons are detected through their conversion in a xenon-methane filled drift chamber immediately following each dielectric stack. By measuring the arrival time of the electron clusters and the total collected charge, the TRD can effectively discriminate between electrons (large $\gamma$ ) and slower moving hadrons (figure 1.3).

\subsubsection{The Do Forward-Backward Drift Chambers}

The forward-backward drift chambers (FDC) are situated on either side of the central tracking chamber. They allow for charged particle tracking in the region from $\eta=1.0$ down to $\eta=3.0$. Each FDC is actually a combination of two structurally different detectors - a $\Phi$ layer sandwiched in between two $\theta$ layers. The $\Phi$ layer consists of a series of drift chambers oriented to give the azimuthal coordinate of particle tracks whereas the $\theta$ layers give the polar angle with respect to the beam line.

\subsection{The Do Calorimeter}

The DO calorimeter is a uranium liquid argon sampling calorimeter which is divided into a central calorimeter (CC) and two endcap calorimiters (EC). Each of the three detector sections is contained within a seperate cryostat (figure 1.4). The central calorimeter consists of three seperate layers - an electromagnetic (EM), fine hadronic $(\mathrm{FH})$ and coarse hadronic $(\mathrm{CH})$ section. 
Electromagnetic showers originating from electrons, photons, or neutral pions are fully contained within the EM section, whereas, strongly interacting particles will leave little energy in the EM section. Most of the energy produced by the strongly interacting particles is deposited in the $\mathrm{FH}$ with some leakage into the $\mathrm{CH}$.

The EM section is subdivided azimuthally into 32 modules with each module longitudinally seperated into 4 sections of $2,2,7$, and 10 radiation lengths $\left(X_{0}\right)$ respectively. The 4 layers span a total of .76 absorption lengths ( $\lambda$ ). The transverse segmentation is $\Delta \eta=\Delta \phi=.1$ except for the third EM section which has $\Delta \eta=\Delta \phi=.05$ and corresponds to the region of maximum energy deposition. The FH consists of 16 seperate modules subdivided longitudinally into 3 seperate sections of $1.3,1.0$, and $0.9 \lambda$. Transverse segmentation is $\Delta \eta=\Delta \phi=.1$ for each layer. The $\mathrm{CH}$ section consists of a single layer of 16 modules with the same segmentation as the $\mathrm{FH}$, however, the uranium absorbers are replaced by much thicker copper absorbers $(\lambda \approx 4)$. A detailed description of the central and endcap calorimeters can be found in several sources, 7,8

A high energy particle entering the calorimeter will interact either electromagnetically or strongly with the dense absorber material (uranium or copper). The resulting spray of secondary particles ionizes the liquid argon contained in the gap between absorber plates. The ionization is then collected by pads residing on a signal board in the gap. Figure 1.5 shows the arrangement of absorber material and charge collection pads for a calorimeter module. An electric field is maintained in the liquid argon gaps by placing a high voltage onto the resistive coat surrounding the signal board and the copper charge collection pads. The absorber plates are kept at ground. The ionization electrons drift toward the pads and generate a signal proportional to the incident energy of the particle. 


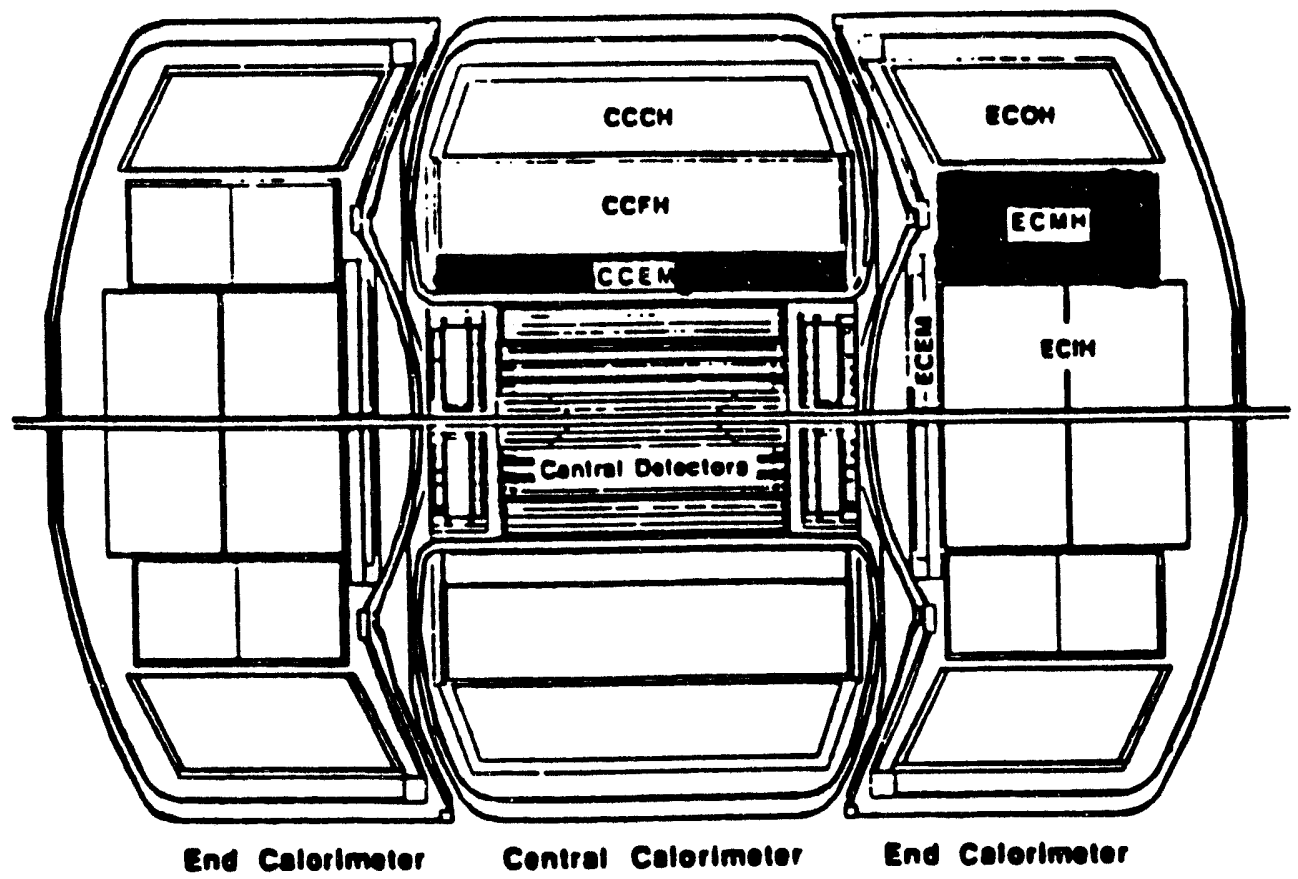

Figure 1.4: Cross section of the Do calorimeter showing the central and endcap sections. The central region consists of electromanetic (CCEM), fine hadronic (CCFH), and coarse hadronic (CCCH) sections. The endcaps contain electromagnetic (ECEM), inner hadronic (ECIH), middle hadronic (ECMH), and outer hadronic (ECOH) sections. 


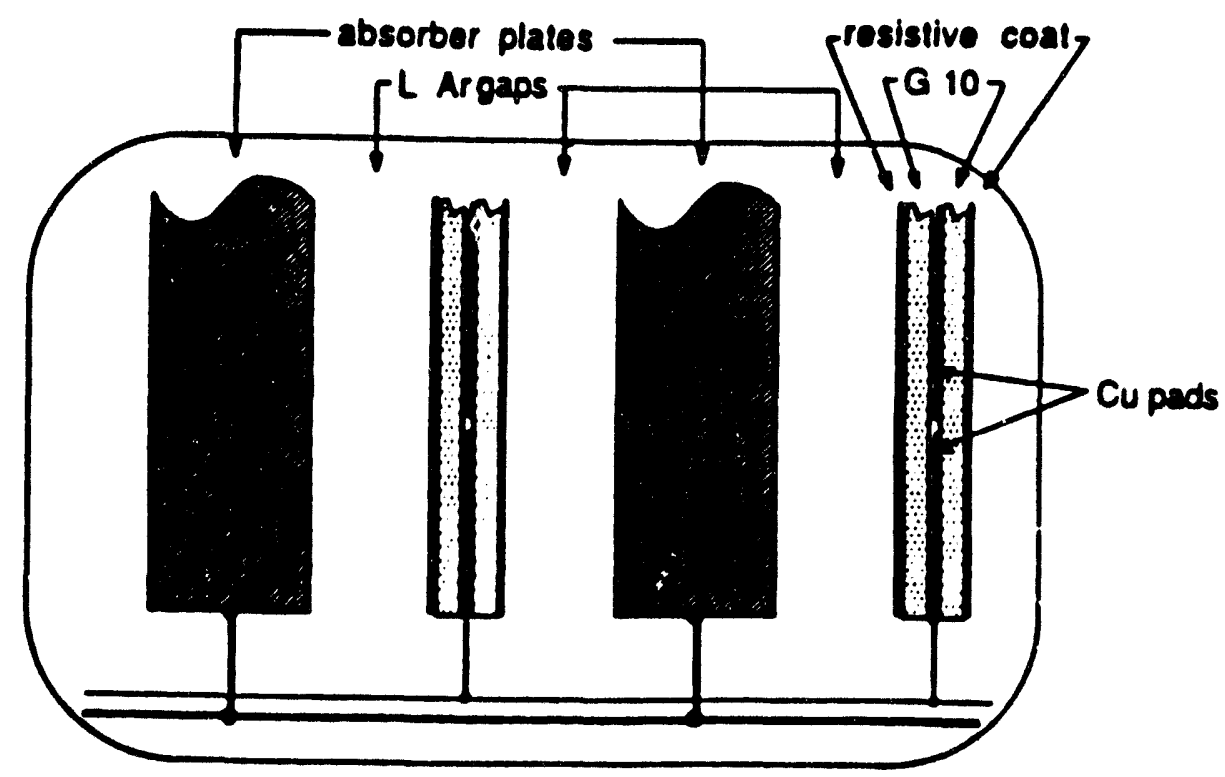

Figure 1.5: Cross section of a calorimeter module showing the arrangment of absorber, liquid argon gaps, and signal boards.

A considerable part of the secondary particles produced in an hadronic cascade within the aborber are $\pi^{\circ} \mathrm{s}$. These $\pi^{\circ} \mathrm{s}$ will interact electromagnetically without any further hadronic contribution. Since the number of secondary $\pi^{\circ} s$ produced fluctuates greatly on an event by event basis, it is important to design a calorimeter which has an equal response to both the electromagnetic and hadronic components of the shower. In this way, the energy resolution becomes insensitive to these $\pi^{\circ}$ fluctuations. Test results on an ECHM module using incident electrons and charged pions in the energy range between 10 and $150 \mathrm{GeV}$ indicate a linear response to generally better than $1 \%$ for both the CCEM and ECMH sections. The ratio of electromagnetic to hadronic response $(e / h)$ is found to be independant of incident energy and is given by $e / h=1.029 \pm .008 .^{9}$ A summary of the energy and position resolution of the Do calorimeter is given in table 1.1. The position resolution results reflect a 
measurement made with $50 \mathrm{GeV}$ electrons for the CCEM and $50 \mathrm{GeV}$ charged pions for the ECMH modules. ${ }^{9}, 10$

\begin{tabular}{|c|c|c|c|}
\hline \multicolumn{4}{|c|}{$\begin{array}{l}\text { Do Calorimeter Energy } \\
\text { and Position Resolution }\end{array}$} \\
\hline Section & $\sigma_{E} / E$ & & $\sigma_{x}$ \\
\hline CCEM & $\approx 17 \% / \sqrt{E}$ & $e$ & $<2 m m$ \\
\hline ECEM & $\approx 16 \% / \sqrt{E}$ & $e$ & \\
\hline ECMH & $\approx 24 \% / \sqrt{E}$ & $e$ & \\
\hline & $\approx 50 \% / \sqrt{E}$ & $\pi$ & $<1.5 \mathrm{~cm}$ \\
\hline ECIH & $\approx 45 \% / \sqrt{E}$ & $\pi$ & \\
\hline
\end{tabular}

Table 1.1. Energy and position resolution of the DO calorimeter.

\subsection{The Muon Detector}

Muon detection (figure 1.6) in D0 is done by tracking in the region between the calorimeter and the magnetized steel toroids and then again after the toroids. The central toroids allow for large angle muon coverage from $45^{\circ} \leq$ $\theta \leq 135^{\circ}$. Two end toroids extend the muon coverage down to $11^{\circ}$ and the small angle muon system (SAMUS) completes the muon acceptance down to $3.7^{\circ}$.

Tracking is done via a series of proportional drift tubes (PDTs) which give muon position measurements before and after the primary bend plane ( $R \theta$ plane). Each PDT contains a single sense wire in addition to charge sensitive cathode pads situated on either side of the wire. The pads, in conjunction with sense wire charge division, allow for muon tracking in the non bend plane $(R \Phi)$, whereas, sense wire drift time information gives the muon position along 


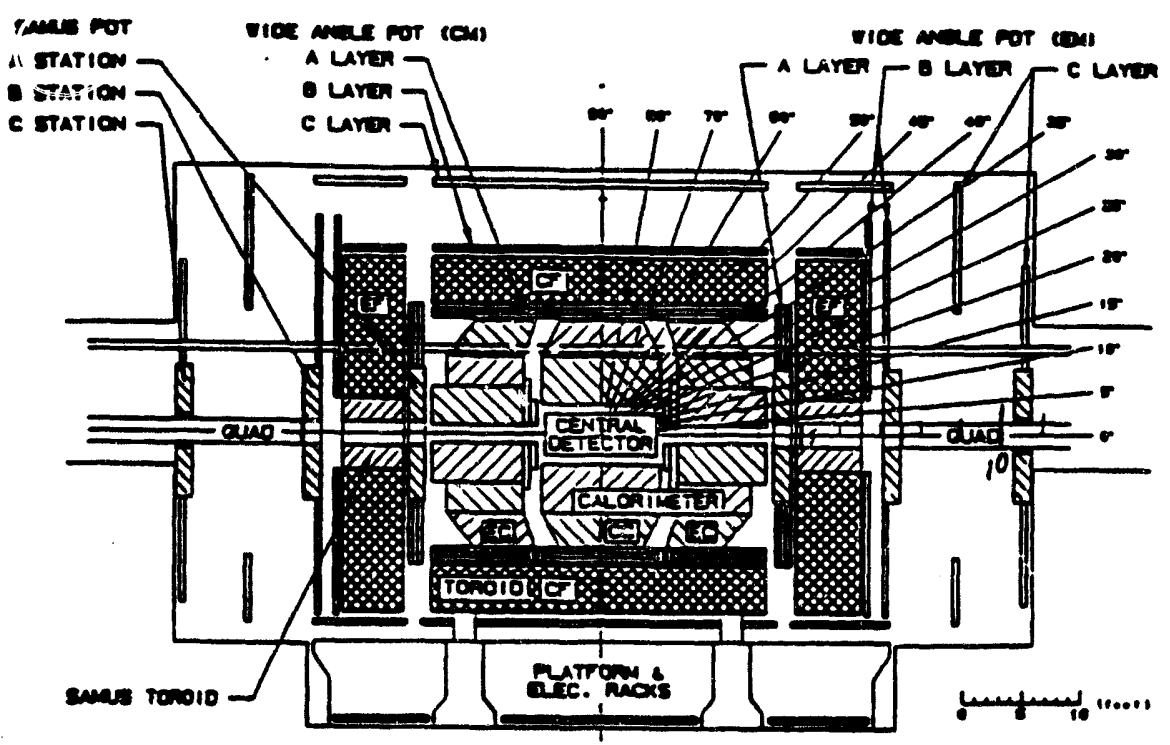

Figure 1.6: Bend plane cross section of the DO muon detector.

the primary bend plane. The PDT resolution is $200 \mu \mathrm{m}$ in the bend plane and $3 \mathrm{~mm}$ in the non bend plane. ${ }^{6}$

The region between the calorimeter and the toroids contains a single 4 layer plane of PDTs. After the toroids, tracking is done via two additional sets of PDT planes each con aining 3 layers. Muon momentum is determined by measuring the deflection in the $R \theta$ plane after passage through the magnetized toroids. The fractional transverse momentum resolution of $\delta P_{T} / P_{T} \approx 18 \%$ for muons with $P_{T} \leq 300 \mathrm{GeV}$ is limited by multiple coulomb scattering within the steel. Above this $P_{T}$, the finite position resolution of the PDTs causes the fractional momentum resolution to deteriorate. 


\section{Chapter 2}

\section{The Central Drift Chamber Mechanics and Signal Processing}

1

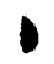

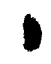

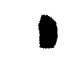




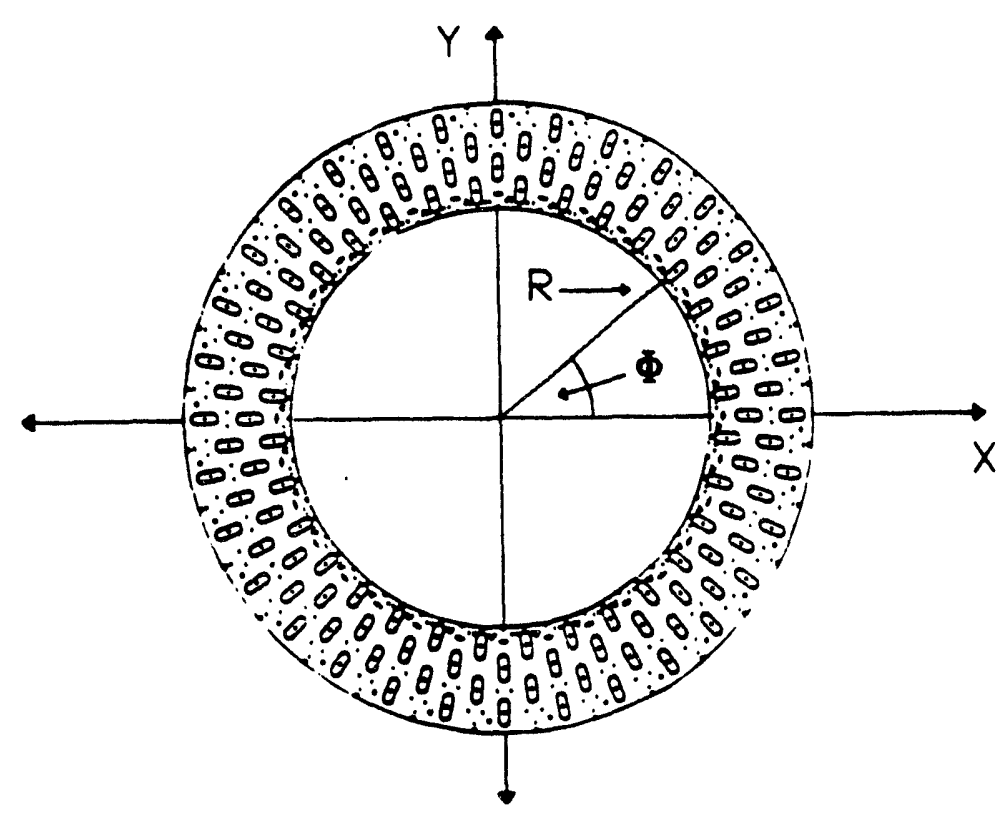

Figure 2.1: $R \Phi$ cross section of the Stony Brook central tracking chamber.

\subsection{The Stony Brook Central Tracking Chamber}

The main design goals of the Stony Brook central drift chamber are to construct a compact detector with excellent track reconstruction capability, good spatial and ionization $(d E / d X)$ resolution, and the ability to distinguish between nearby tracks (two-track resolution). The final design allows tracking from about 50 to $70 \mathrm{~cm}$ radially from the beam pipe and a full $360^{\circ}$ coverage around the interaction region (figure 2.1 ). Tracking along the beam pipe $(Z)$ is limited to $\pm 90 \mathrm{~cm}$ from the nominal interaction point (figure 2.2).

What follows is an overview of the mechanics and field shaping of the central drift chamber. A detailed account of the chamber construction and electrostatics is given in Ties Behnke's doctorial thesis. ${ }^{11}$ 


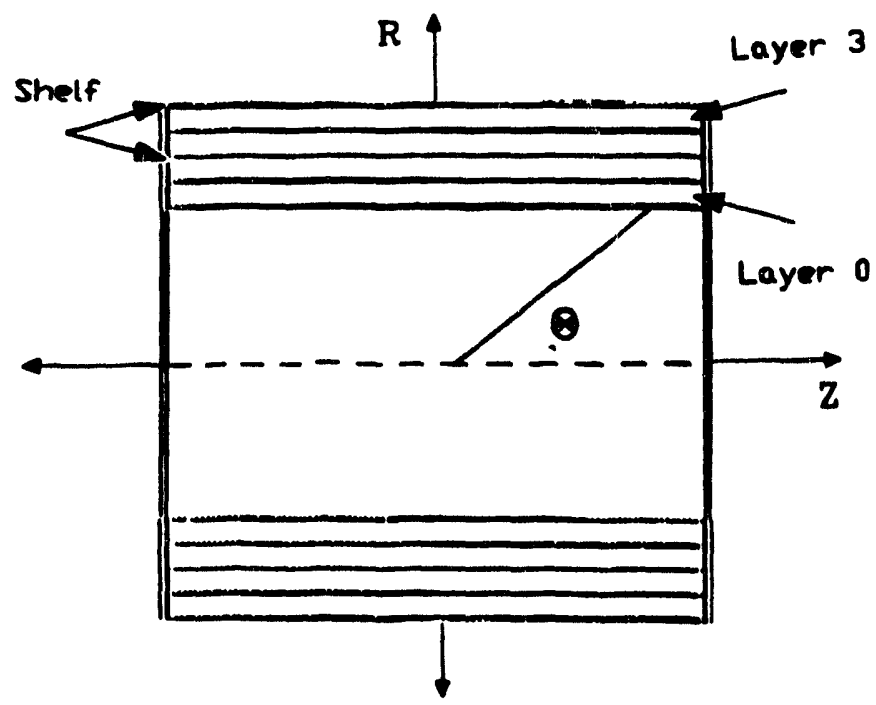

Figure 2.2: $R Z$ cross-section of the completed Stony Brook central tracking chamber.

\subsubsection{Mechanics of the CDC}

The complete central drift chamber is segmented in $\Phi$ by 32 modules with each module being further segmented radially into a staggered set of 4 individual cells (layers), each of which is a self contained drift cell. Each drift cell contains 7 anode or "sense" wires ( $R \Phi$ coordinates) 14 potential wires, and 2 delay lines ( $Z$ coordinate) running along the length of the cell with each delay line adjacent to one of the outer sense wires. The sense wires are connected to both sides of the module via plastic end plugs from which physical access to the sense wire and delay line signals is possible.

The basic building blocks of each module include five shelves, four septa and two end plates (figure 2.3). The shelves are composed of a rigid Rohacell foam core sandwiched by a Kapton-Kevlar-Epoxy skin giving a lightweight yet strong support for the module. Printed directly onto the shelves are graphite 


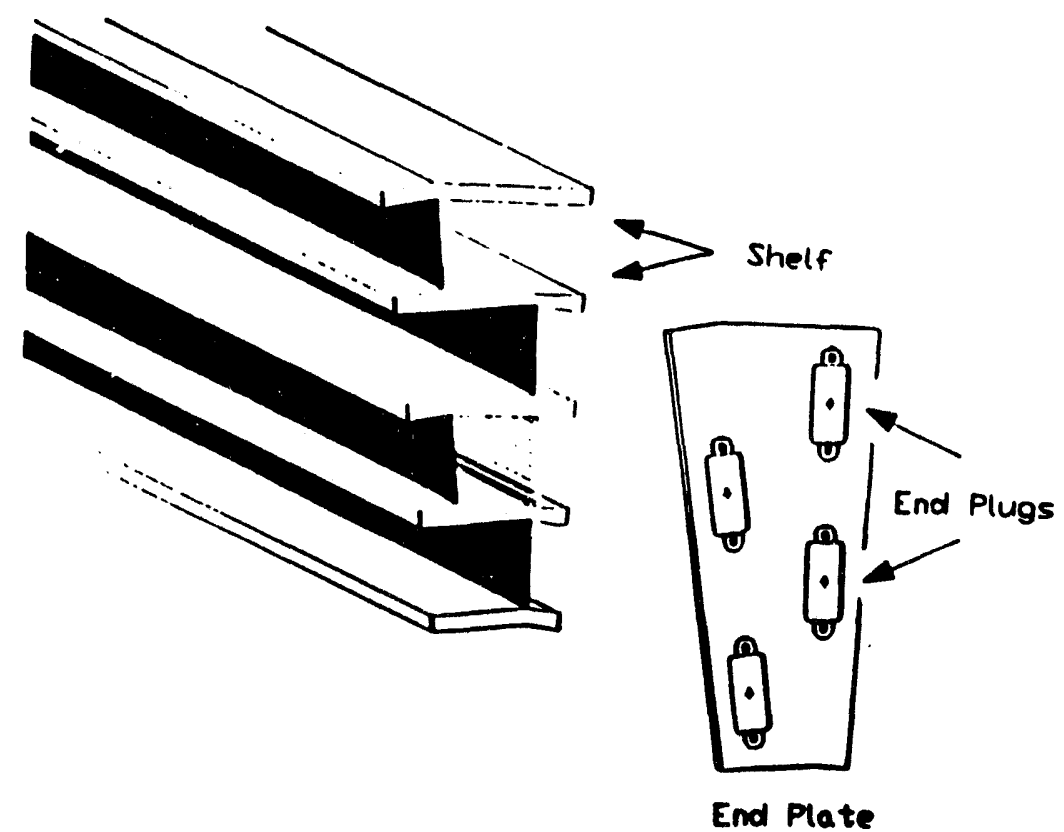

Figure 2.3: A single module as seen from a cutaway of the end plate.

based conducting strips which form the field shaping cage (figure $2.4^{11}$ ). Structurally, the shelves divide the module into layers and also house the delay lines. The septa consist of a $1 \mathrm{~mm}$ sheet of Rohacell core enclosed by sheets of fiberglass and Kapton. The septa plane is rotated with respect to the sense wire plane by 5.625 degrees and provides the physical boundary between succesive sectors while also serving as the cathode of the field cage. A complete module consists of 28 sense wires and 8 delay lines enclosed on both sides by $1 / 4$ in thick end plates to provide stability and support for final mounting of the modules within the external Aluminum support cylinder.

\subsubsection{Field Shaping Within the Drift Cell}

Four groups of potentials are supplied to the drift cell in order to generate the field cage: separate positive high voltages to the inner and outer sense wires, a cathode voltage fed in through the top of each septum, and a central 


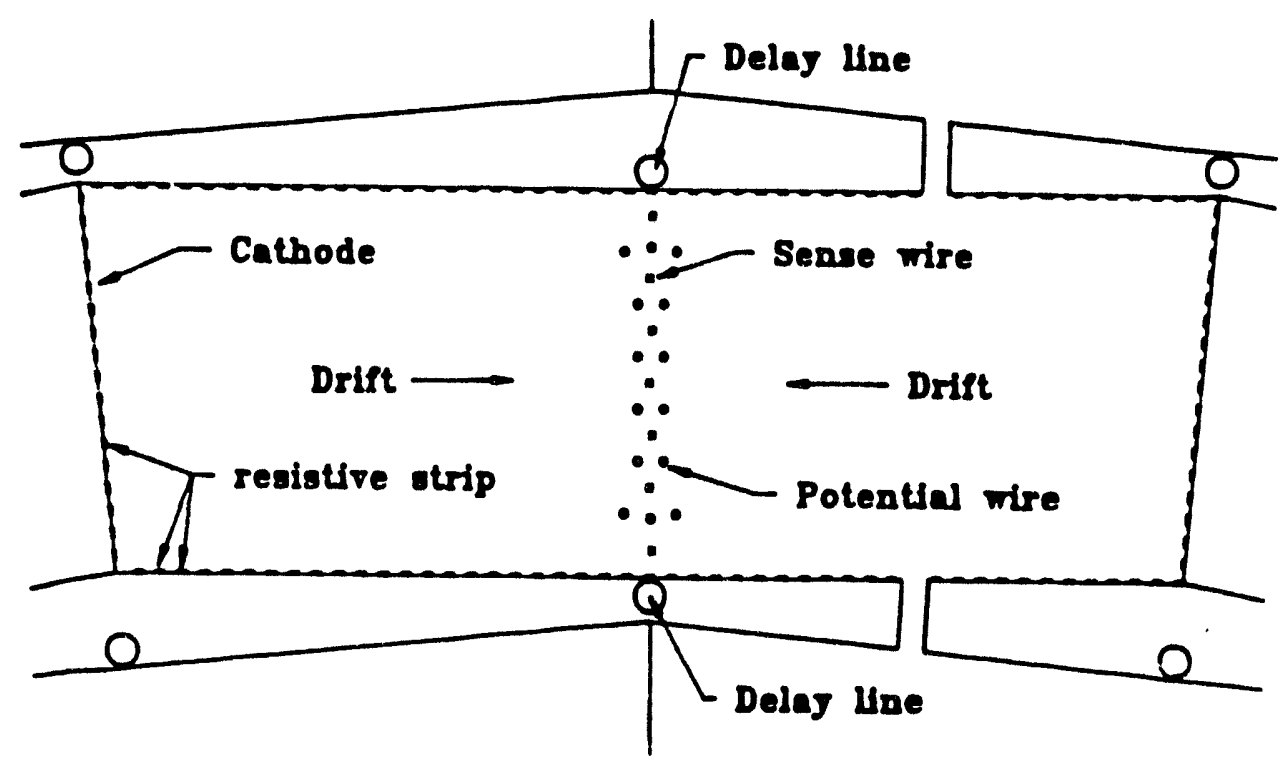

Figure 2.4: $R \Phi$ crossection showing major components of the drift cell.

strip voltage applied to the strip located directly on top of the delay line (figure $\left.2.5^{11}\right)$. The sense wire voltages are fed in on one side of the chamber while the other side connects the sense wire output via a coupling capacitor to the preamp mounted on the outside of the Aluminum support endplate.

The potential wires (figure 2.4 ) near the anodes serve two main purposes:

1. Improve the field shaping in the high field region near the sense wires.

2. Minimize signal induction between adjacent sense wires, and shield the delay lines from pulses induced by the near-outer wires (crosstalk).

The delay line output is an induced signal which is generated by the outer sense wires. Under normal running conditions, the outer sense wires are driven at a higher voltage ( $\approx 10 \%$ greater) than the inner sense wires to insure an adequate delay line response. The 3 potential wires near the outer sense wires provide additional shielding from inner wire signal induction onto the delay 


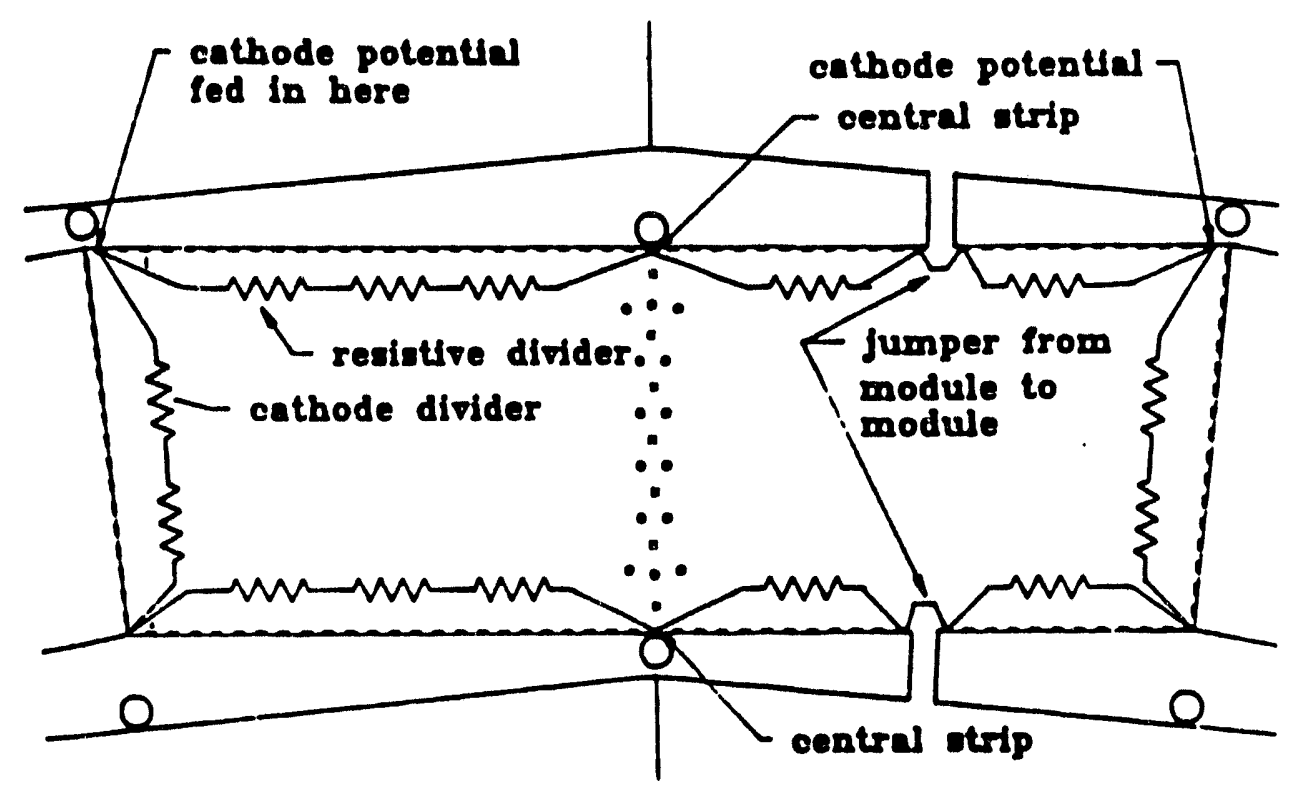

Figure 2.5: Schematic of the field shaping elements of the drift cell.

line. Another important aspect of the potential wires is their ability to modify the near anode electric field (gain) without having to change the applied sense wire voltage. If a sense wire should begin discharging against one of the adjacent potential wires, the addition of a small positive voltage ( 300 Volts) onto the potential wires will inhibit the anode wires from entering a state of continuous discharge. Otherwise, the potential wires are nominally kept at ground.

A uniform field cage is generated by using a voltage divider network to step the input cathode voltage down to the central strip voltage by a constant amount through the conductive strips painted on the shelves (figure 2.5) The cathode voltage is also stepped down along the septa strips to compensate for the widening drift space as one moves radially outward within the cell. Figure $2.6^{11}$ shows the resulting electric field configuration within the drift cell. The vertical lines are the electric field and the horizontal lines spaced $30 \mathrm{~ns}$ apart 


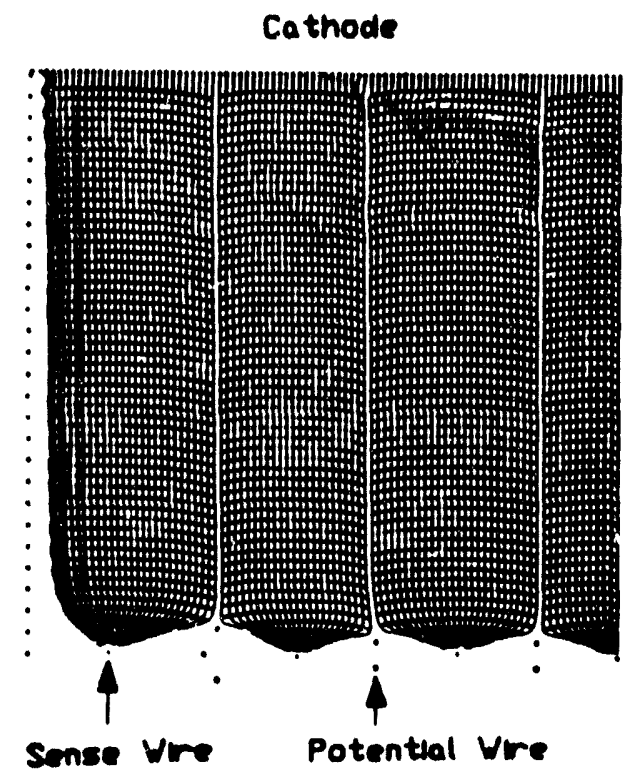

Figure 2.6: Electric field configuration within a drift cell.

represent lines of equal drift time, that is, electrons which begin the drifting process along one of these lines will arrive at the anode simultaneously.

\subsection{Signal Processing and Data Acquisition}

This section gives a brief description of the major components of the Do central tracking chamber electronics along with a description of the data aquisition chain currently under development at Fermilab.

\subsubsection{Electronics Overview}

Analog signals generated from the sense wires and delay lines are amplified by a Fujitsu charge sensitive preamplifier ${ }^{12}$ mounted directly on the endplate of the central tracking chamber's Aluminum support cylinder. The amplified pulses are then sent to the shaper inputs (figure 2.7 ). The primary 
Chapter 2: The Central Drift Chamber Mechanics and Signal Processing

21

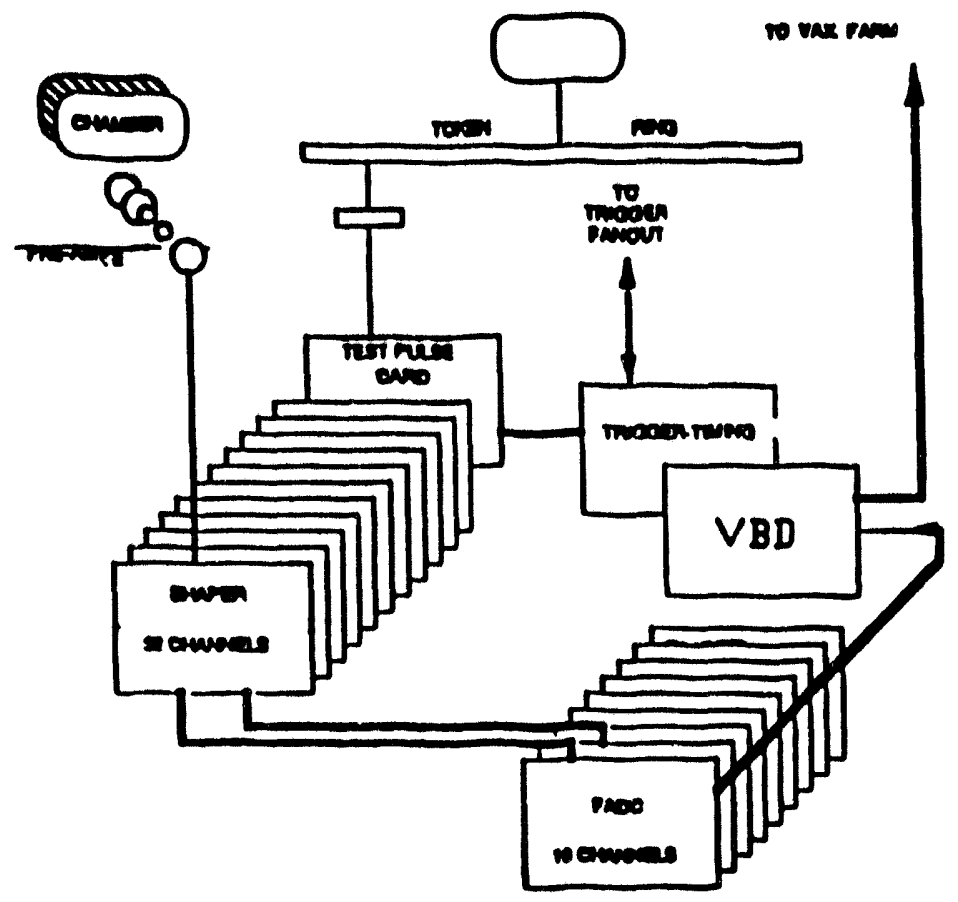

Figure 2.7: Layout of signal processing chain.

function of the shaper is to differentiate the input pulse and cut off the long signal tails induced on the sense wires and delay lines by the slow process of positive ion drift away from the chamber anode (sense wire) toward the cathode. This filtering through of only the main part of the signal is essential for resolving multiple pulses which are near in time, thereby, improving the individual particle track reconstruction in events which contain many tracks in a small region of space.

After shaping, signals are then sent to a Fast Analog to Digital Coverter (FADC). The FADC contains three main data processing stages ${ }^{13}$ (fig 2.8): Analog input processing, conversion from analog to digital, and suppression of digitized data which does not contain pulse information. The first stage consists of a bilinear amplifier. Shaper signals entering the FADC are first subjected to a user controlled adjustable gain input stage. Output from this stage then enters the bilinear amplifier. Low input signals result in a high 


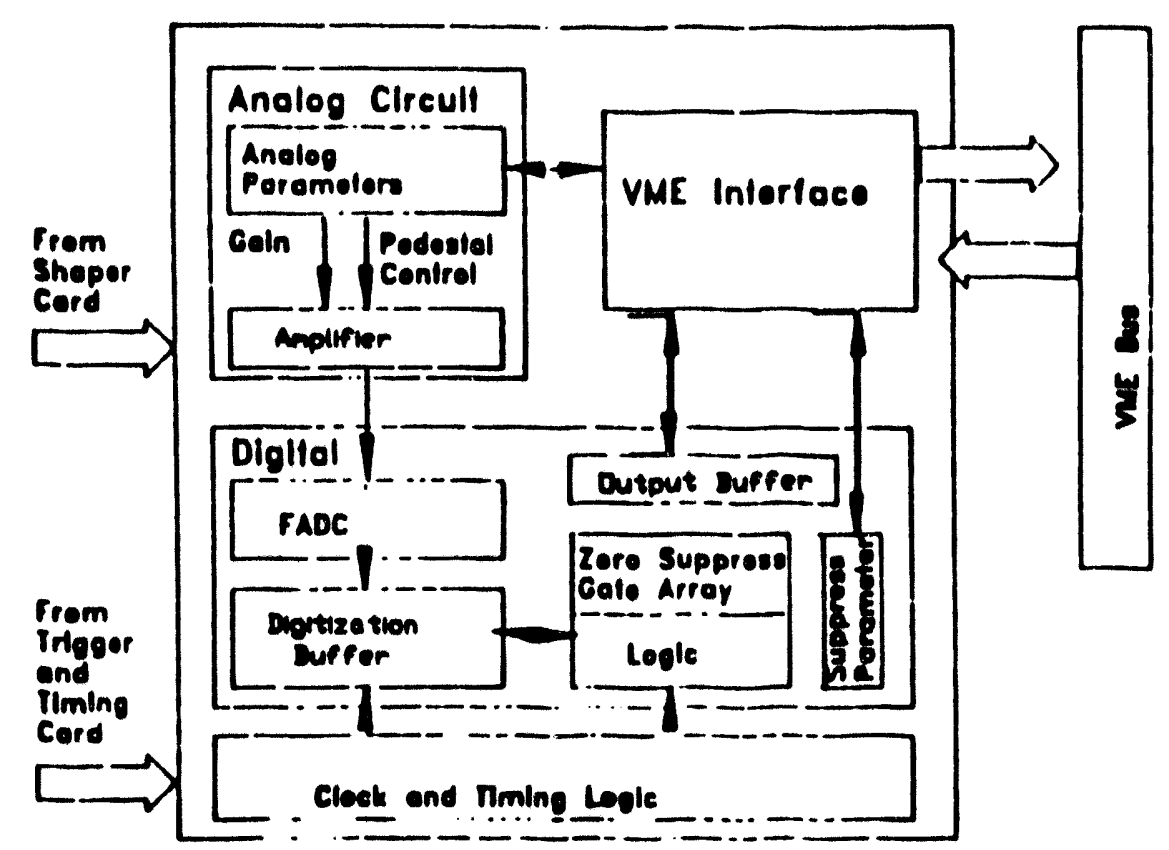

Figure 2.8: Signal processing stages of FADC card.

amplification while larger signals get amplified by about a factor of 12 relative to the small signals. This bilinear design enables a larger spread of input voltages to be digitized before saturating the 8 bit dynamic range of the FADC.

Timing of the FADC modules is provided by two clocks. A master clock provides the $106 \mathrm{Mhz}$ signal which drives the digitization of the analog input every 9.4 nanoseconds. A seperate START clock signal synchronized to the Tevatron proton-antiproton beam crossing arrives at the FADC just prior to the chamber pulses and is responsible for initiating the digitizatization cycle.

Once the digitization buffer is filled (maximum of 512 / 8 bit words), a trigger signal sent from the Level 1 trigger framework ${ }^{14}$ to the trigger control card $^{15}$ (TCC) tells the data acquisition electronics that the digitized data represents a valid event. The data buffer is then filtered through the FADC zero suppression chip (section 2.2 .2 ) and all digitizations not containing pulse 
information are discarded. The compressed data is then loaded into the FADC output buffer and sent over a 32 bit wide path (VME bus) to the buffer driver (VBD). From there the data is sent to a dual port memory buffer which can be accessed by an available VAX 4000 computer node (level $2^{16}$ ). The level 2 nodes format and write the data onto disk for eventual offline processing.

\subsubsection{Hardware Zero Suppression}

The DO zero suppression chip residing on the FADC cards enables fast and efficient isolation of true signals by suppressing digitized data which fail certain absolute value and differential comparison criteria (see below). Depending on each channel's baseline fluctuation, thresholds are chosen to effectively eliminate random noise or strangely shaped pulses which, in all probability, do not represent a true signal. Refering to figure 2.9 , let $B_{i}$ represent the digitized pulse height in the $i^{\text {th }}$ time slice for a particular channel and let $D_{i}=B_{i}-B_{i-1}$. The threshold flags $F_{1}$ through $F_{4}$ and the differential flags $F_{3}$ and $F_{6}$ are set true or false based on the conditions

$$
\begin{aligned}
& F_{1}=B_{i-1} \text { and. } B_{i} \text { and. } B_{i+1}>T_{1} \\
& F_{2}=B_{i-1} \text { and. } B_{i} \text { and. } B_{i+1} \geq T_{2} \\
& F_{3}=B_{i-1} \text { and. } B_{i} \text { and. } B_{i+1}<T_{3} \\
& F_{4}=B_{i-1} \text { and. } B_{i} \text { and. } B_{i+1}<T_{4} \\
& F_{5}=D_{i} \text { and. } D_{i+1}>S_{1} \\
& F_{6}=S_{2}<\left(D_{i} \text { and. } D_{i+1}\right)<S_{3}
\end{aligned}
$$

A signal is isolated by passing a leading edge $(L E)$ and a trailing edge (TE) criteria given by,

$$
\begin{aligned}
& L E=\left(F_{1} \text {.and. } F_{5}\right) \text {.or. } F_{2} \\
& T E=\left(F_{3} \text { and. } F_{6}\right) \text {.or. } F_{4}
\end{aligned}
$$


with thresholds $T_{1}$ through $T_{4}$ and differences $S_{1}$ through $S_{3}$ typically chosen to be

$$
\begin{aligned}
T_{i} & =\langle\text { Baseline }\rangle_{i}+N_{i} \sigma_{i} \\
S_{i} & =K_{i} \sigma_{i}
\end{aligned}
$$

where $<$ Baseline $\rangle_{i}$ is the average DC level for the $i^{\text {th }}$ channel and $\sigma_{i}$ is the random DC level fluctuation. $N_{i}$ and $K_{i}$ are user adjustable constants optimized for isolating true signals and rejecting unwanted noise. Data which pass both the $L E$ and $T E$ criteria are buffered into the FADC back-end memory and await transfer to the level 2 dual port memory. If only the $L E$ criterion is satisfied within a maximum string length, the data string is accepted and buffered as before.

\subsubsection{Electronic Calibration}

The relative signal path delays for each channel of the electronics chain is monitored using a special test pulse circuitry card mounted in the shaper crate (figure 2.7). The pulse amplitude can be remotely programmed by the user via token ring (IBM standard communication protocol). The test pulse may be sent into the chamber mounted preamplifiers, or bypass the preampamplifiers, and sent directly into the shaper inputs. In this way, signal transmission problems arising between the preamplifier and shapers can be isolated from those arising further down the electronics chain.

\subsection{Software Hitfinding}

The software hitfinder searches for pulses by subjecting the hardware zero suppressed data to an additional set of threshold and difference criteria. Again referring to figure 2.9 , the leading edge $L E_{\text {oft }}$ of a pulse candidate is defined 
by

$$
L E_{\text {ooft }}=\left\{\begin{array}{c}
D_{i-1} \text {.and. } D_{i} \text {.and. } D_{i+1}>\Delta_{1} \\
\text { or. } \\
\left(D_{i-1} \text {. and. } D_{i}\right)>\Delta_{1} \text {.and. }\left(B_{i}-B_{i-2}\right)>\Delta_{2}
\end{array}\right.
$$

where $\Delta_{1}$ and $\Delta_{2}$ are user adjustable differences optimized to include only signals which originate from true ionizing events. As a final criterion, the peak amplitude of the pulse (after baseline subtraction) is constrained to lie above some threshold.

The arrival time for signals which pass the software zero suppression is determined by finding the weighted center of gravity of the differential of the rising edge. The arrival time $T$ is given by:

$$
T=\frac{\sum_{i=2}^{n} i W_{i-1} D_{i}}{\sum_{i=2}^{n} W_{i-1} D_{i}}
$$

with the sum terminated when $D_{i}<0$, or equivalently, at the start of the pulse's trailing edge. The weight factor $W_{i}$ is given by $C * i$ with $\mathrm{C}$ chosen to optimize the timing resolution of the sense wire and delay line signals. The trailing edge cutoff $T E_{\text {ooft }}$ of the pulse is simply determined by the inverse of the leading edge condition

$$
T E_{\text {ooft }}=\left\{\begin{array}{c}
D_{i-1} \text {.and. } D_{i} \text {.and. } D_{i+1}<-\Delta_{1} \\
\text { or. } \\
\left(D_{i-1} \text {. and. } D_{i}\right)<-\Delta_{1} \text {.and. }\left(B_{i}-B_{i-2}\right)<-\Delta_{2}
\end{array}\right.
$$

If while searching for the trailing edge of a pulse the hitfinder encounters the leading edge of a second pulse, an overlap flag is set and the first pulse is truncated at the point where the second pulse begins. If the pulse trailing edge fails to meet the cutoff requirements within some maximum number of 
time slices after the peak, the pulse is truncated and the hitfinder resumes its search for new pulses. 

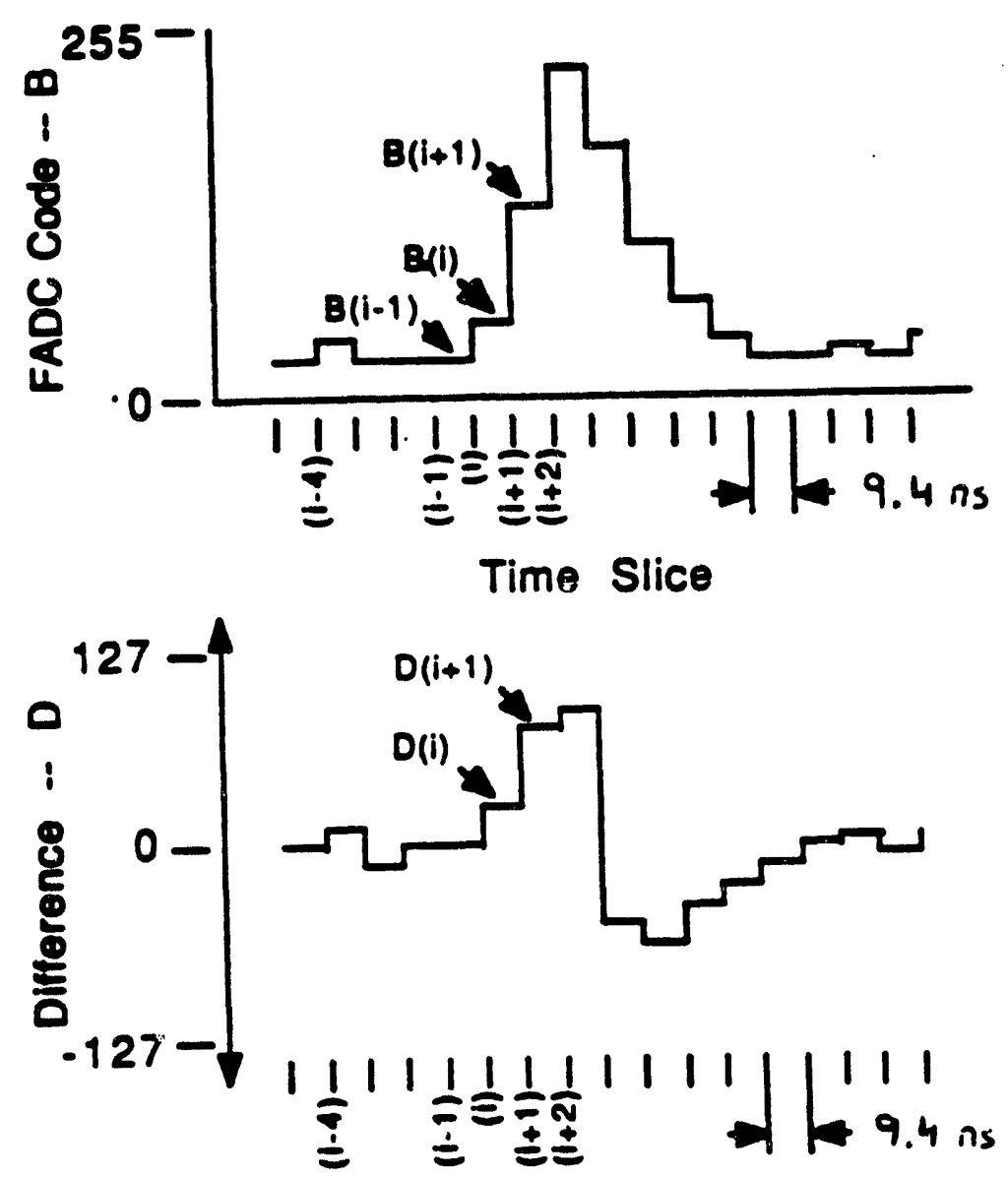

Figure 2.8: Hardware zero suppression of raw FADC data. 


\section{Chapter 3}

\section{Theory of Electron Drift in Gases}




\subsection{Transport Theory of Electron Drift in Gases}

The theory of electron drift in gases is based on a solution of the Boltzmann transport equation. If one considers the distribution function $F(r, v, t)$ such that $F(r, v, t) d r d v$ is the number of electrons contained within the phase space volume element $d \mathbf{r} d \mathbf{v}=d x d y d z d v_{x} d v_{y} d v_{z}$ having a velocity $\mathbf{v}$ and occupying spatial location $r$ at time $t$, then according to Liouville's theorm

$$
\frac{d F}{d t}=\frac{\partial F}{\partial t}+\frac{\partial F}{\partial \mathbf{r}} \frac{d \mathbf{r}}{d t}+\frac{\partial F}{\partial \mathbf{v}} \frac{d \mathbf{v}}{d t}=0
$$

which states that the number of electrons contained inside a given volume of phase space remains constant. An immediate consequence is that the total energy and momentum entering a phase space volume element through electron drift and diffusion must equal the energy and momentum lost due to collisions with the gas molecules.

\subsubsection{Electron Energy Distribution and Drift Velocity}

Following the work of $\mathrm{H}$. A. Lorentz, ${ }^{17}$ the form of the distribution function applicable to electron drift in gases was arrived at by Morse, Allis, and Lamar ${ }^{18}$ by considering a uniform electric field $E$ oriented along the $x$ axis. In this case, $F$ is isotropic in the $y z$ plane and independent of $x$ and, therefore, only a function of the electron velocity $v$ and $\cos \theta$ where $\theta$ is the angle of the electron velocity vector measured with respect to the $x$ axis. It was assumed that $F$ could be expanded in series of Legendre polynomials $P_{k}(\cos \theta)$

$$
F(v, \theta)=F_{0}(v)+\sum_{k=1}^{\infty} F_{k}(v) P_{k}(\cos \theta)
$$

The population of electrons with a velocity between $v$ and $v+d v$ is then given by

$$
\begin{aligned}
N_{e}(v) & =v^{2} d v \int_{0}^{2 \pi} \int_{0}^{\pi}\left[F_{0}+\sum_{k=1}^{\infty} F_{k} P_{k}(\cos \theta)\right] \sin \theta d \theta d \phi \\
& =4 \pi v^{2} d v F_{0}(v)
\end{aligned}
$$


with the $k \geq 1$ terms identically zero due to the general property

$$
\int_{0}^{\pi} P_{k}(\cos \theta) \sin \theta d \theta=0
$$

The factor $4 \pi v^{2} d v$ in result 3.3 is the volume in velocity space of a thin shell of radius $v$ and thickness $d v$, therefore, $F_{0}(v)$ gives the number density of electrons with velocity $v$.

The average instantaneous velocity in the $x$ direction ( $E$ field direction) for electrons contained within a $(v, d v)$ shell is by definition

$$
\begin{aligned}
\left\langle V_{x}\right\rangle(v) & =\frac{1}{N_{e}(v)} v^{2} d v \int_{0}^{2 \pi} \int_{0}^{\pi}\left[F_{0}+\sum_{k=1}^{\infty} F_{k} P_{k}(\cos \theta)\right](v \cos \theta) \sin \theta d \theta d \phi \\
& =\frac{1}{N_{e}(v)} 4 \pi v^{3} d v \int_{0}^{\pi} F_{1} \cos ^{2} \theta d \theta \\
& =\frac{v F_{1}(v)}{3 F_{0}(v)}
\end{aligned}
$$

Result 3.5 was obtained by substituting 3.3 and using 3.4 along with $P_{1}(\cos \theta)=$ $\cos \theta$ and the orthogonality condition

$$
\int_{0}^{\pi} P_{j}(\cos \theta) P_{k}(\cos \theta) \sin \theta d \theta=\left[\frac{2}{2 j+1}\right] \delta_{j k}
$$

Equations 3.3 and 3.5 show that only the first two terms in the Legendre expansion of $F$ are sufficient to describe the density and motion of electrons with energy $\epsilon=\frac{1}{2} m v^{2}$ for the case considered here.

\subsubsection{Elastic Collisions}

Beginning with $F$ expressed in terms of electron energy $\epsilon$

$$
F(\epsilon, \theta)=F_{0}(\epsilon)+\cos \theta F_{1}(\epsilon)
$$

and applying the constraints imposed by 3.1, Morse, Allis, and Lamar ${ }^{18}$ derived two coupled equations for $F_{0}$ and $F_{1}$

$$
\begin{aligned}
e E \frac{\partial}{\partial \epsilon}\left(v F_{0}\right)-\frac{2 e E}{m v} F_{0} & =-\frac{v F_{1}}{l_{e}(\epsilon)} \\
\frac{e E}{3} \frac{\partial}{\partial \epsilon}\left(v F_{1}\right) & =\Lambda(\epsilon) \frac{\partial}{\partial \epsilon}\left(\frac{\epsilon v F_{0}}{l_{e}(\epsilon)}\right)
\end{aligned}
$$


where $e$ is the electron charge, $m$ is the electron mass, $E$ is the electric field, $l_{e}(\epsilon)$ is the mean free path length of the electrons between two elastic collisions with the gas molecules, and $\Lambda(\epsilon)$ is the mean fractional energy lost by the electron after collision. For the special case of an elastic collision with a stationary molecule of mass $M \gg m$, classical two body kinematics gives $\Lambda=2 m / M$ to first order in $m / M$. In general, however, $\Lambda$ must be considered a function of the electron energy $\epsilon$ since the addition of polyatomic gases with vibrational and rotational energy states (such as methane and carbon dioxide) will lead to non elastic collisions (section 3.2.1) and modify the value of $\Lambda$.

Equation 3.8(3.9) equates the amount of momentum(energy) gained through diffusion and drift in the $E$ field with the loss of momentum(energy) due to collisions. Combining 3.8 and 3.9, we arrive at the differential equation governing the electron number density distribution function $F_{0}$

$$
\frac{2(e E)^{2}}{3 m} \frac{\partial}{\partial \epsilon}\left[\epsilon l_{e}(\epsilon) \frac{\partial\left(F_{0} / v\right)}{\partial \epsilon}\right]+\Lambda(\epsilon) \frac{\partial}{\partial \epsilon}\left(\frac{\epsilon v F_{0}}{l_{e}(\epsilon)}\right)=0
$$

The above equation for $F_{0}$ assumes that the mean electron energy is much greater than the thermal energy of the gas molecules $\left(\frac{3}{2} k T \approx .04 \mathrm{eV}\right.$ at room temperature) so that the gas molecules could be considered at rest before and after the collision. Taking into account the thermal motion of the gas, equation 3.10 becomes $^{19}$

$$
\frac{\partial}{\partial \epsilon}\left(\left[\frac{2(e E)^{2}}{3 m} \epsilon l_{e}(\epsilon)+\frac{2 \Lambda(\epsilon) \epsilon^{2}}{m l_{e}(\epsilon)} k T\right] \frac{\partial\left(F_{0} / v\right)}{\partial \epsilon}+\frac{\Lambda(\epsilon) \epsilon v F_{0}}{l_{e}(\epsilon)}\right)=0
$$

with $T$ the temperature of the gas and $k$ the Boltzmann constant. Integrating once and rearranging the coefficients gives

$$
\left[\left(e E l_{e}(\epsilon)\right)^{2}+3 \Lambda(\epsilon) \epsilon k T\right] \frac{\partial F_{0} / v}{\partial \epsilon}+\frac{3 m v \Lambda(\epsilon)}{2} F_{0}=0
$$

Assuming an exponential solution $C e^{-\alpha(\epsilon)}$ for $F_{0}$ and substituting into 3.12 gives a differential equation for $\alpha(\epsilon)$

$$
\frac{\partial \alpha}{\partial \epsilon}=\frac{3 \Lambda(\epsilon) \epsilon}{\left(e E l_{e}(\epsilon)\right)^{2}+3 \Lambda(\epsilon) \epsilon k T}-\frac{1}{2 \epsilon}
$$


which is easily integrated to give the form of the distribution function

$$
F_{0}(\epsilon)=C \sqrt{\epsilon} \exp \left(-\int \frac{3 \Lambda(\epsilon) \epsilon d \epsilon}{\left[e E l_{e}(\epsilon)\right]^{2}+3 \Lambda(\epsilon) \epsilon k T}\right)
$$

where the constant $C$ is adjusted for normalization such that $\int F_{0}(\epsilon) d \epsilon=1$

If the electron energy gained by drifting in the field $E$ between elastic collisions is small $\left[e E l_{e}(\epsilon)\right]^{2} \ll 3 \Lambda(\epsilon) \epsilon k T$ then the electron energy distribution must be in equilibrium with that of the gas and equation 3.14 correctly reduces to the Maxwell distribution

$$
F_{0}(\epsilon)=C \sqrt{\epsilon} \exp (-\epsilon / k T)
$$

The mean drift velocity of the electrons along the $E$ field direction $w_{x}$ is by definition

$$
w_{x}=\int_{0}^{\infty} v \cos \theta F(\epsilon, \theta) d \epsilon
$$

and can be shown to be related to $F_{0}$ through the expression ${ }^{20}$

$$
w_{x}=\frac{-2 e E}{3 m} \int_{0}^{\infty} \epsilon l_{e}(\epsilon) \frac{\partial\left(F_{0}(\epsilon) / v\right)}{\partial \epsilon} d \epsilon
$$

\subsection{Application to Polyatomic and Multi Component Gas Mixtures}

The specific experimental requirements for the successful operation of a drift chamber such as a low working voltage, high electron multiplication near the anode (gain), fast recovery, and long lifetime dictate the use of a multi component gas mixture. Electrons liberated by a charged particle traversing the gaseous volume of a drift chamber will migrate toward the positively charged anodes. As they approach the high electric field region near the anode ( $\approx 50 \mu m$ from the wire center), they acquire the energy necessary to ionize the gas molecules resulting in a rapid electron multiplication. A noble gas such 
as argon is generally used as the primary gas component since electron multiplication occurs at much lower fields than in complex polyatomic molecules ${ }^{21}$ thereby allowing a low voltage operation. Polyatomic gases such as methane and $\mathrm{CO}_{2}$, on the other hand, have many non ionizing vibrational and rotational energy states which do not contribute to high gain operation. During the electron multiplication process, excited and ionized noble gas atoms are formed. The excited atoms return to the ground state by emission of a photon with an energy ( $\approx 12 \mathrm{ev}$ for argon) usually well above the ionization potential of the materials contained within the drift cell. These photons can then extract additional electrons which drift toward the anode and initiate a new electron avalanche soon after the primary multiplication. The ionized atoms produced during the initial avalanche drift towards the cathode and are neutralized by extracting electrons from the cathode. The energy balance is maintained either by photon emission or secondary emission of an additional electron from the cathode surface. The probability of both of these processes is high enough that a permanent condition of discharge is likely.

This unwanted continuous discharge operation can be suppressed by adding a polyatomic gas with a large amount of non radiative excited states in the energy range of the deexcitation photons. Methane, for example, is very efficient in absorbing photons in the range $\approx 8$ to $15 \mathrm{eV}$ which includes the energy range of photons produced by argon deexitation. The excited polyatomic molecules dissipate their excess energy through either elastic collisions or by dissociation into less complex structures. A complete discussion of gases and their effects on drift chamber operation may be found in the article by $F$. Sauli'in 


\subsubsection{Polyatomic Gases (Inelastic Collisions)}

The results of section 3.1.2 may be extended to include the effects of inelastic collisions between electrons and polyatomic gases. The general relationship between the mean free path length between collisions $l(\epsilon)$ and the collision cross section $\sigma(\epsilon)$ for a given electron energy $\epsilon$ is given by

$$
\sigma(\epsilon)=\frac{1}{N l(\epsilon)}
$$

where $N$ is the Loschmidt number defined as

$$
N=N_{0} \frac{P}{760} \frac{273}{T}
$$

with $N_{0}=2.687 \times 10^{25}$ molecules $/ \mathrm{m}^{3}, P$ the gas pressure, and $T$ the gas temperature.

If the excitation(inelastic) cross section for a polyatomic molecule is not negligible compared to the elastic scattering cross section for a given electron energy $\epsilon$, then it becomes likely that one or more of these states may become excited during a collision. In this way, the electron will on average lose more energy per collision than in the purely elastic case. The additional fractional energy loss per collision due to the existence of excited states with energy $\epsilon_{h}$ (in the approximation that the inelastic cross section $\sigma_{h}(\epsilon)$ is much less than the elastic cross section $\left.\sigma_{e}(\epsilon)\right)$ is

$$
\left.\frac{\Delta \epsilon}{\epsilon}\right|_{\text {inelastic }}=\sum_{h} \frac{\epsilon_{h} \sigma_{h}(\epsilon)}{\epsilon \sigma_{e}(\epsilon)}
$$

where the sum extends over all states which can be excited given the electron energy $\epsilon$. The total fractional energy loss per collision then becomes

$$
\Lambda(\epsilon)=\frac{2 m}{M}+\sum_{h} \frac{\epsilon_{h} \sigma_{h}(\epsilon)}{\epsilon \sigma_{e}(\epsilon)}
$$

where, as mentioned in section $3.1 .2,2 m / M$ is the iractional energy loss for elastic scattering. 


\subsubsection{Electron Diffusion}

As a result of multiple collisions with the gas molecules, a swarm of electrons originally confined to a small volume will diffuse while drifting in an electric field. The spatial extension of the electron swarm $\sigma_{*}$ along the $E$ field direction after drifting for a time $t$ is ${ }^{21}$

$$
\sigma_{2}=\sqrt{2 D t}
$$

where $D$ is the diffusion coefficient given by ${ }^{20}$

$$
D=\frac{1}{3} \int_{0}^{\infty} l_{e}(\epsilon) v F_{0}(\epsilon) d \epsilon
$$

and $v=\sqrt{2 \epsilon / m}$ is the instantaneous velocity of the electron.

\subsubsection{Multicomponent Gases}

Consider an $n$ component gas mixture with $p_{i}$ being the fraction of type $i$ gas in the $\operatorname{mix}\left(\sum p_{i}=1\right)$. The important results expressed by equations 3.14 , 3.17 , and 3.23 can be used unmodified for a multi component gas by defining a composite value for $\sigma_{e}(\epsilon)$

$$
\sigma_{e}(\epsilon)=\sum_{i} p_{i} \sigma_{e i}(\epsilon)
$$

where $\sigma_{e i}(\epsilon)$ is the elastic scattering cross section for the $i$ th gas. Similiarly, a composite $\Lambda(\epsilon)$ may be defined as

$$
\Lambda(\epsilon)=\frac{1}{\sigma_{e}(\epsilon)} \sum_{i} p_{i} \sigma_{e i}(\epsilon) \Lambda_{i}(\epsilon)
$$

with $\sigma_{e}(\epsilon)$ given by 3.24 and $\Lambda_{i}(\epsilon)$ as defined by 3.21 . The mean free path length for elastic collisions $l_{e}(\epsilon)$ in equation 3.14 is found with the aid of 3.18 and remembering that the composite cross section of equation 3.24 must be used. 
Note that in all equations involving the mean free path, only the elastic mean free path approximation was used, however, inelastic effects are taken into account in the computation of the electron energy distribution function $F_{0}(\epsilon)$ (equation 3.14 ) via equations 3.21 and 3.25 . 


\section{Chapter 4}

\section{$R \Phi$ Tracking}




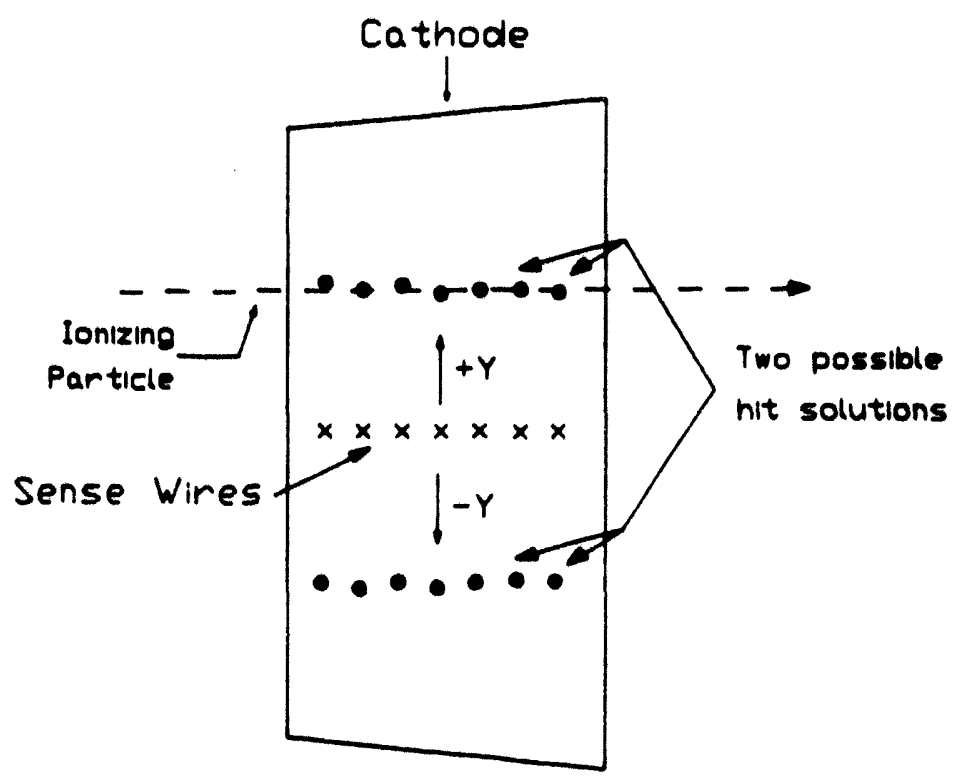

Figure 4.1: Two possible coordinates of a sense wire hit within a single drift cell.

\subsection{Segment Finding}

The arrival time of each sense wire generated pulse cannot be uniquely associated with one and only one space coordinate within a single drift cell (fig 4.1) since it is not known on which side of the sense wire the ionizing event occurred. Two "trial hits" are initially generated per sense wire pulse, one in the $+Y$ and one in the $-Y$ region of the drift cell. The hit coordinates are then transformed from the local drift cell frame to the DO frame assuming ideally known chamber geometry constants.

A track segment (track frum a single drift cell) is defined by associating hits within a cell based on their $R, \Phi$ position relative to a road defined by the outer most hits. Both $R$ and $\Phi$ are measured with respect to the D0 coordinate frame. Referring to figures $4.2 a$ and $b$, the slope of the road is given by

$$
M_{\text {road }}=\frac{\Phi_{l}-\Phi_{f}}{R_{l}-R_{f}}
$$


and can be constrained by a user defined cut on $\left|\Phi_{l}-\Phi_{f}\right|$. The difference between the expected and actual $\Phi$ coordinate of an intermediate hit is

$$
\Delta \xi=\Phi_{f}+M_{\text {road }}\left(R_{\text {hit }}-R_{f}\right)-\Phi_{\text {hit }}
$$

It is important to note that the validity of equation 4.2 depends on the approximation that a straight line in the $X Y$ plane will transform into a straight line in the $R \Phi$ plane. This approximation is valid provided that $R_{l}-R_{f} \ll R_{f}$ and is satisfied by tracks originating from the DO interaction region.

An intermediate hit is picked up as part of a segment candidate if $|\Delta \xi| \leq$ $|\delta \phi|$ where

$$
\delta \phi \approx \frac{\Delta D}{R_{f}}
$$

and $\Delta D$ is a variable parameter suitably picked by the user such that $|\Delta D| \ll$ $\boldsymbol{R}_{f}$. For cases in which there is more than one hit on an intermediate wire, the one with the smallest $\Delta \xi$ is chosen. A minimum of 2 to 3 intermediate hits out of a possible 5 are generally required to pass the above cuts before a segment candidate is accepted.

Once a segment candidate has been found, it is paramaterized by minimizing the function (figure 4.3)

$$
\chi^{2}=\sum_{i=1}^{n}\left(\left(X_{i}-X 0\right) \sin \Phi-\left(Y_{i}-Y 0\right) \cos \Phi\right)^{2} / \delta_{i}^{2}
$$

with respect to $X \theta, Y 0$ and $\Phi . X_{i}$ and $Y_{i}$ are the coordinates for the $i$ th hit, $\Phi$ is the angle of the segment in the $X Y$ plane with respect to the $X$ axis, and $n$ is the number of hits in the segment. The derivatives with respect to $X 0$ and $Y 0$ give the minimization conditions

$$
\sum_{i=1}^{n}\left(\left(X_{i}-X 0\right) \sin \Phi-\left(Y_{i}-Y 0\right) \cos \Phi\right) / \delta_{i}^{2}=0
$$

and the derivative with respect to $\Phi$ gives 

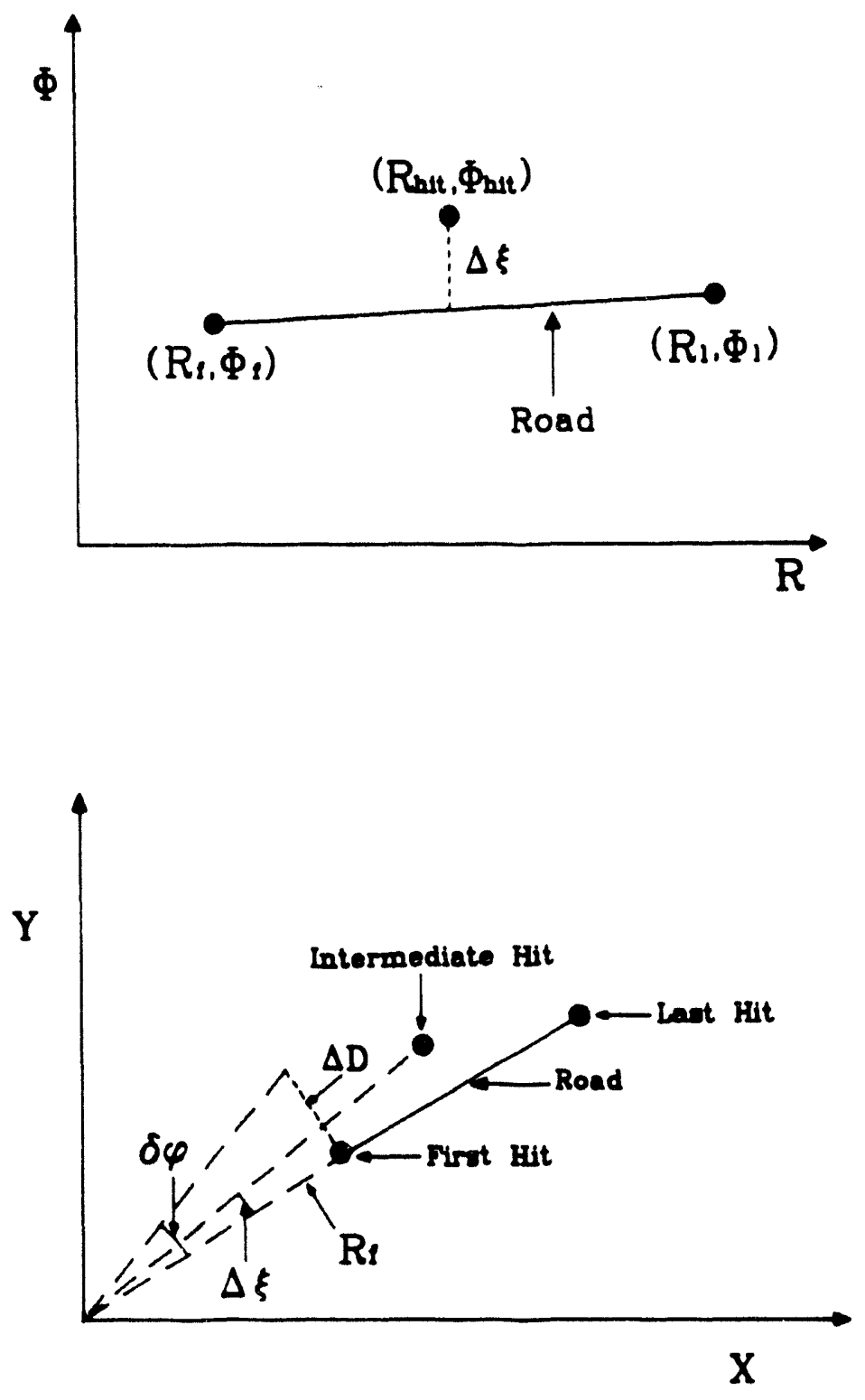

Figure 4.2: Association of sense wire hits within a segment candidate in the $R \Phi$ plane (not to scale). 


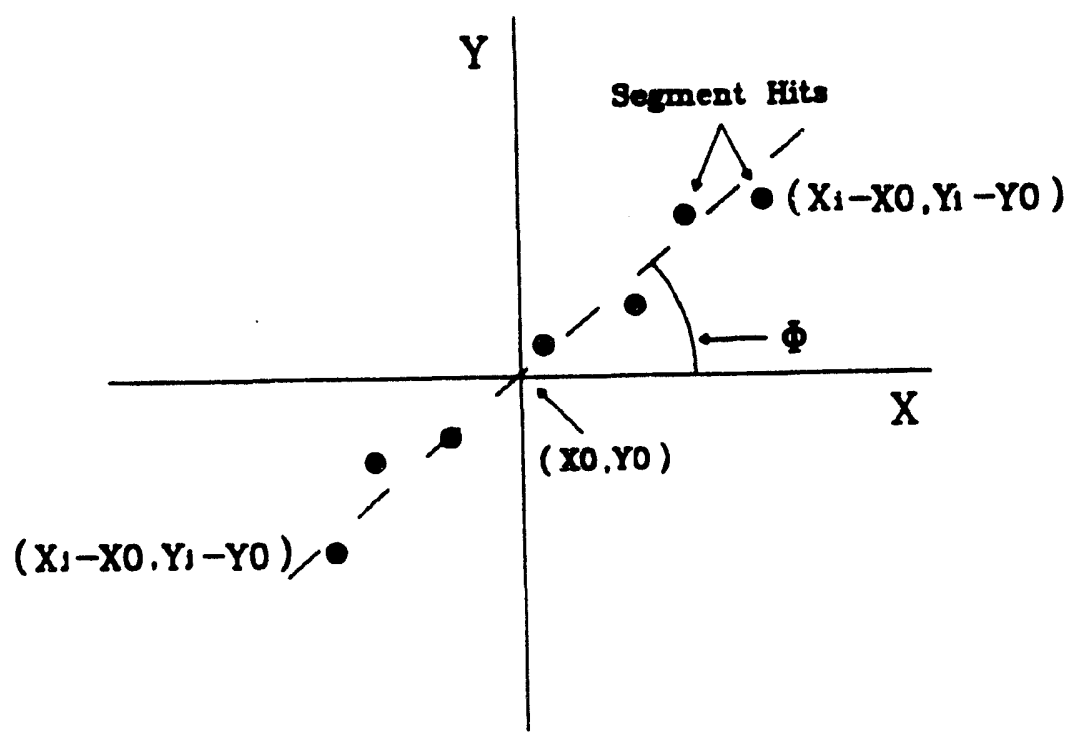

Figure 4.3: Parameterisation of segment fit.

$$
\begin{aligned}
& \frac{1}{2} \sin 2 \Phi \sum_{i=1}^{n}\left(\left(X_{i}-X 0\right)^{2}-\left(Y_{i}-Y 0\right)^{2}\right) / \delta_{i}^{2}- \\
& \left.\cos 2 \Phi \sum_{i=1}^{n}\left(X_{i}-X 0\right)\left(Y_{i}-Y 0\right)\right) / \delta_{i}^{2}=0
\end{aligned}
$$

with $\delta_{i}$ the intrinsic sense wire resolution.

Equation 4.5 is satisfied by choosing

$$
\begin{aligned}
& X 0=\sum_{i=1}^{n} \frac{X_{i}}{\delta_{i}} / \sum_{j=1}^{n} \frac{1}{\delta_{i}} \\
& Y 0=\sum_{i=1}^{n} \frac{Y_{i}}{\delta_{i}} / \sum_{j=1}^{n} \frac{1}{\delta_{i}}
\end{aligned}
$$

and solving for $\Phi$ in 4.6 we get

$$
\Phi=\frac{1}{2} \tan ^{-1}\left(\frac{2 \sum_{i=1}^{n}\left(X_{i}-X 0\right)\left(Y_{i}-Y 0\right) / \delta_{i}^{2}}{\sum_{i=1}^{n}\left(\left(X_{i}-X 0\right)^{2}-\left(Y_{i}-Y 0\right)^{2}\right) / \delta_{i}^{2}}\right)
$$

A segment is accepted after passing a $\chi^{2}$ cut and the participating hits are flagged to avoid re-use in subseauent segment finding. 


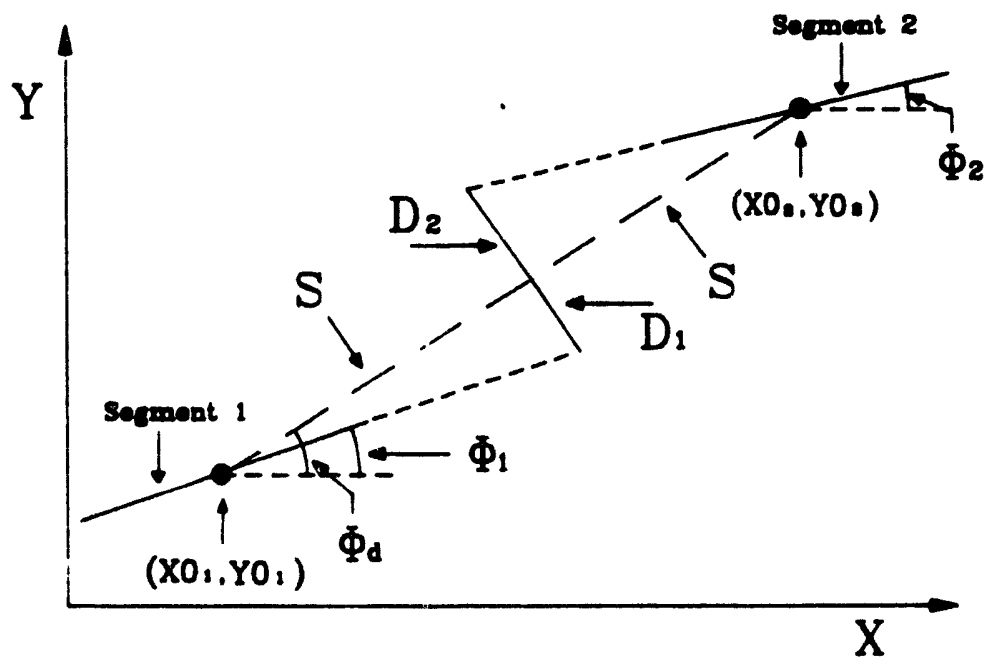

Figure 4.4: Association of two segments as part of a full $R \Phi$ track.

\subsection{Construction of Full $R \Phi$ Tracks}

Once all the segments hrve been found, they must be associated to form full tracks. Figure 4.4 shows two segments in different layers and their corresponding fit parameters $X 0, Y 0$ and $\Phi$. The segment association is done by requiring that the absolute $\Phi$ difference between segments is less than some acceptable value

$$
\left|\Phi_{2}-\Phi_{1}\right| \leq \delta \Phi
$$

and that an extrapolation from each segment to a common midpoint yields an absolute difference that is less than some tolerance

$$
\left|D_{2}-D_{1}\right|=\left|S\left(\tan \left(\Phi_{d}-\Phi_{1}\right)+\tan \left(\Phi_{d}-\Phi_{2}\right)\right)\right| \leq \delta D
$$

where $S=.5 \sqrt{\left(\left(X_{2}-X 0_{1}\right)^{2}+\left(Y 0_{2}-Y 0_{1}\right)^{2}\right)}$. Segments are associated starting from layer 3 and working inward towards layer $u$. A full track is made by 
requiring at least 3 participating segments passing the above cuts. Once a full track candidate is found, its parameters $X 0, Y 0$, and $\Phi$ are determined from equations 4.7 through 4.9 , a $\chi^{2}$ cut is applied, and segments participating in the track are tagged and made ineligible for reuse in subsequent track finding. 


\section{Chapter 5}

\section{CDC Performance Studies}




\subsection{Introduction}

A series of cosmic ray runs were taken on the completed central drift chamber spanning from June to December of 1989 at Stony Brook University. Also, tests of a functional chamber prototype containing two full sectors (the premod) were taken at the Fermilab test beam facility from March to April of 1990. The results of the data analysis are presented here.

\subsection{Cosmic Ray Experimental Setup}

The central drift chamber was mounted within a rotatable housing assembly which allowed seperate sectors of the chamber to be illuminated by cosmic rays. Each run consisted of two full sectors of sense wire and delay line information. Consecutive runs were taken by rotating the housing assembly by one sector thereby requiring 32 runs for the complete chamber. A coincidence between three sets of scintillators, one narrow counter at the center of the chamber and two wide counters below the active sectors, served as the cosmic ray trigger. To minimize multiple scattering within the chamber, low energy particles were rejected by placing iron absorbers between the lower 2 sets of scintillators.

The chamber analog signals were sent to shaper boards via flat coaxial cables and then routed to the flash analog to digital converters (FADC). Values for the FADC zero suppression parameters were downloaded from a Microvax II host computer which also served to format the FADC output and write it onto disk. The FADC zero suppression parameters were chosen to adequately eliminate the baseline data and thereby reduce the event size. A rough event selection criteria specifying a minimum and maximum event size was implimented on the host computer to reject events with too many or too few hits. 


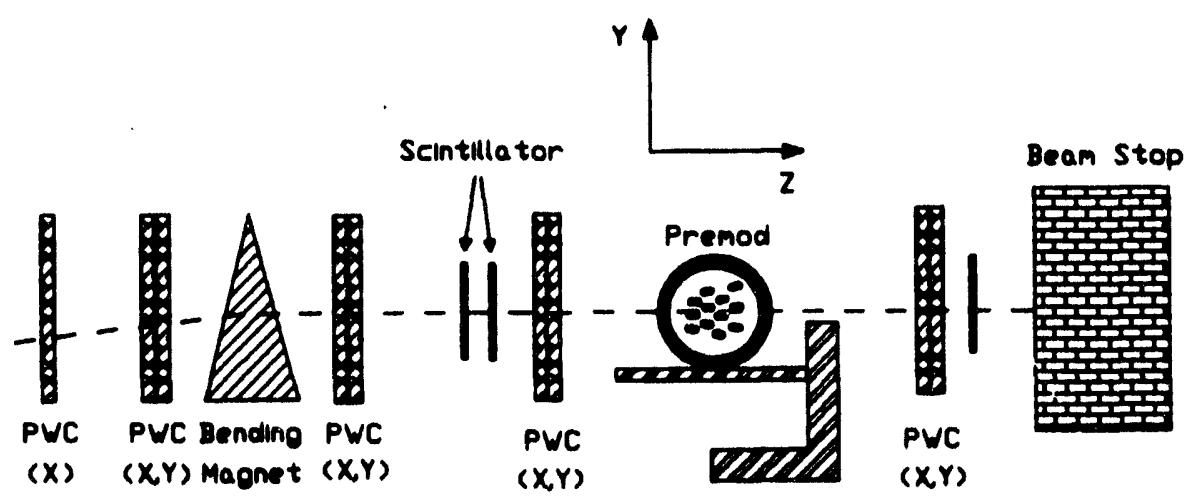

Figure 5.1: Fermilab premod test experimental setup.

\subsection{Fermilab Test Beam Experimental Setup}

The central tracking chamber premod is a functional version of the Do central drift chamber consisting of 3 full sectors and 4 layers of drift cells. It was mounted at the Fermilab test beam facility on top of a precision movable table which allowed accurate placement of the chamber at various positions with respect to the test beam.

The true position of an ionizing event within the premod was determined by tracking across a series of carefully surveyed proportional wire chambers (PWCs) oriented to give the position of the ionizing pion beam at various locations along the beamline. The pion test beam was generated by bombarding a fixed berilium target with protons extracted from the inain ring of the Fermilab tevatron collider. An analyzing magnet selected the $150 \mathrm{GeV}$ pions used as the ionizing beam. Track reconstruction was done using an $\mathrm{X}$ coordi- 
nate measuring PWC located upstream, one set of $\mathrm{X}$ and $\mathrm{Y}$ chambers before and after the momentum selecting bend magnet, and finally, a set of $X$ and $Y$ PWC's before and after the premod (figure 5.1).

Each Fermilab propotional wire chamber (PWC) consisted of an X and $Y$ plane of 64 or 96 wires per plane except the NW8 PWC which had a single 64 wire $\mathrm{X}$ plane. The interwire spacing is $1 \mathrm{~mm}$ except for wire 1 which is displaced an additional $1 / 4 \mathrm{~mm}$. Pulses from the PWC wires were amplified, discriminated, and then dispatched to a series of latch modules which were set ON if the wire associated with the particular latch containes a pulse and if the latch modules received an acknowledgment from the trigger logic that a coincidence of the three scintillation counters flanking the premod had occured.

\subsection{Electronic Delays}

\subsubsection{Cosmic data}

Relative electronic channel delays were determined by simultaneously injecting a test pulse into the active sectors and measuring the relative arrival time into each channel's FADC. Test pulses were sent from the shaper crate backplane, through flat coaxial feeder cables, and into the chamber preamplifiers. From there the test pulses traversed the same electronics path as the signals generated by the chamber itself. Differences in the feeder cable propagation times were measured and included in the final electronic channel delays.

The overall electronic delay is a constant time offset which was determined directly from the cosmic ray data. The distance of an ionizing event from a particular sense wire is given by $D=V_{d}(T-T 0)$ where $V_{d}$ is the electron drift velocity, $T$ is the arrival time of the sense wire pulse as measured from the FADC, and $T O$ is the time offset to be determined which includes the relative 


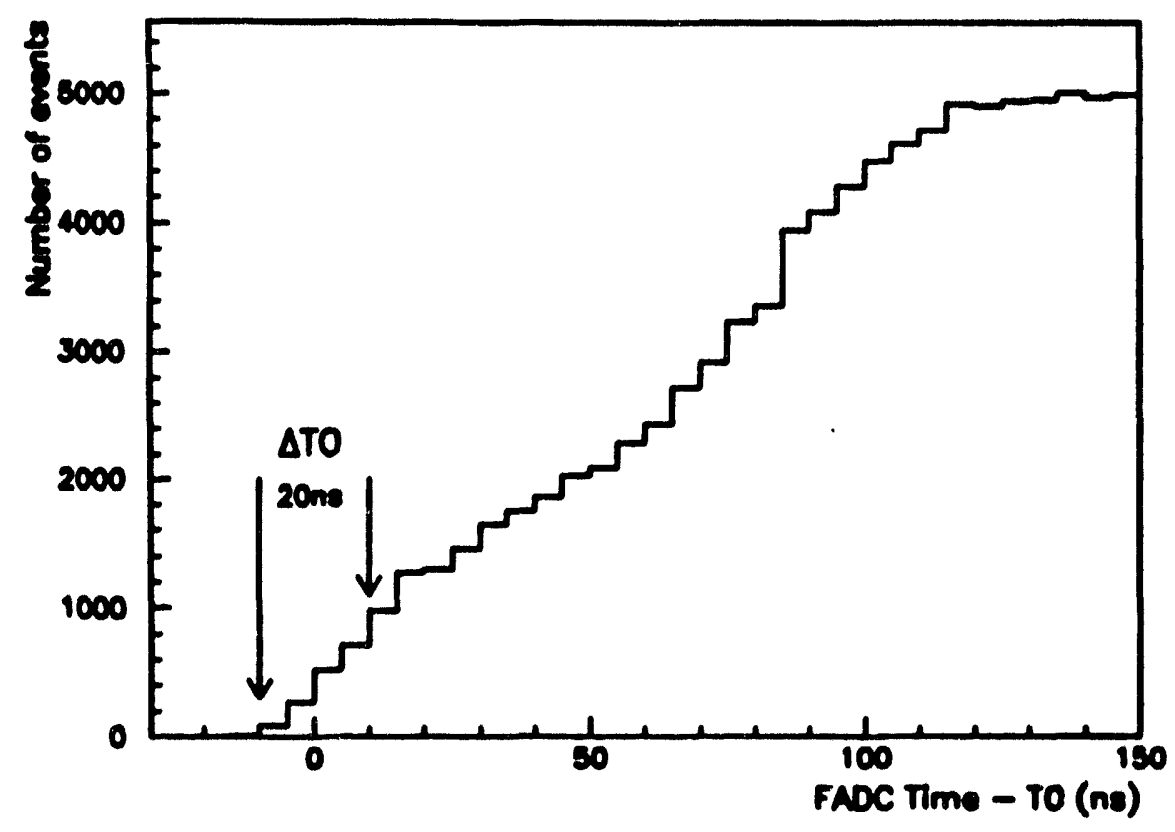

Figure 5.2: Leading edge of electron arrival time distribution.

and overall electronic delay. $T$ is a minimum when an ionizing event occurs directly on the sense wire $(D=0)$, therefore, the overall electronic delay is determined by adjusting the leading edge of the time distribution $T_{\min }$ so that $T_{\min }-T 0=0$ (figure 5.2). The accuracy to which the overall delay can be determined using this method is limited by the reduced chamber efficiency and resolution near the anode. A conservative estimate of the overall electronic delay uncertainty is \pm 10 ns.

\subsubsection{Fermilab Data}

The relative electronic delays for the Fermilab runs were monitored using the test pulse circuitry outlined in subsection 2.2.3. The DO calibration program ${ }^{22}$ determined the electronic delays, mean baselines and baseline fluctuations for each channel and placed the information into a database which allowed a quick and easy association between data runs and corresponding 


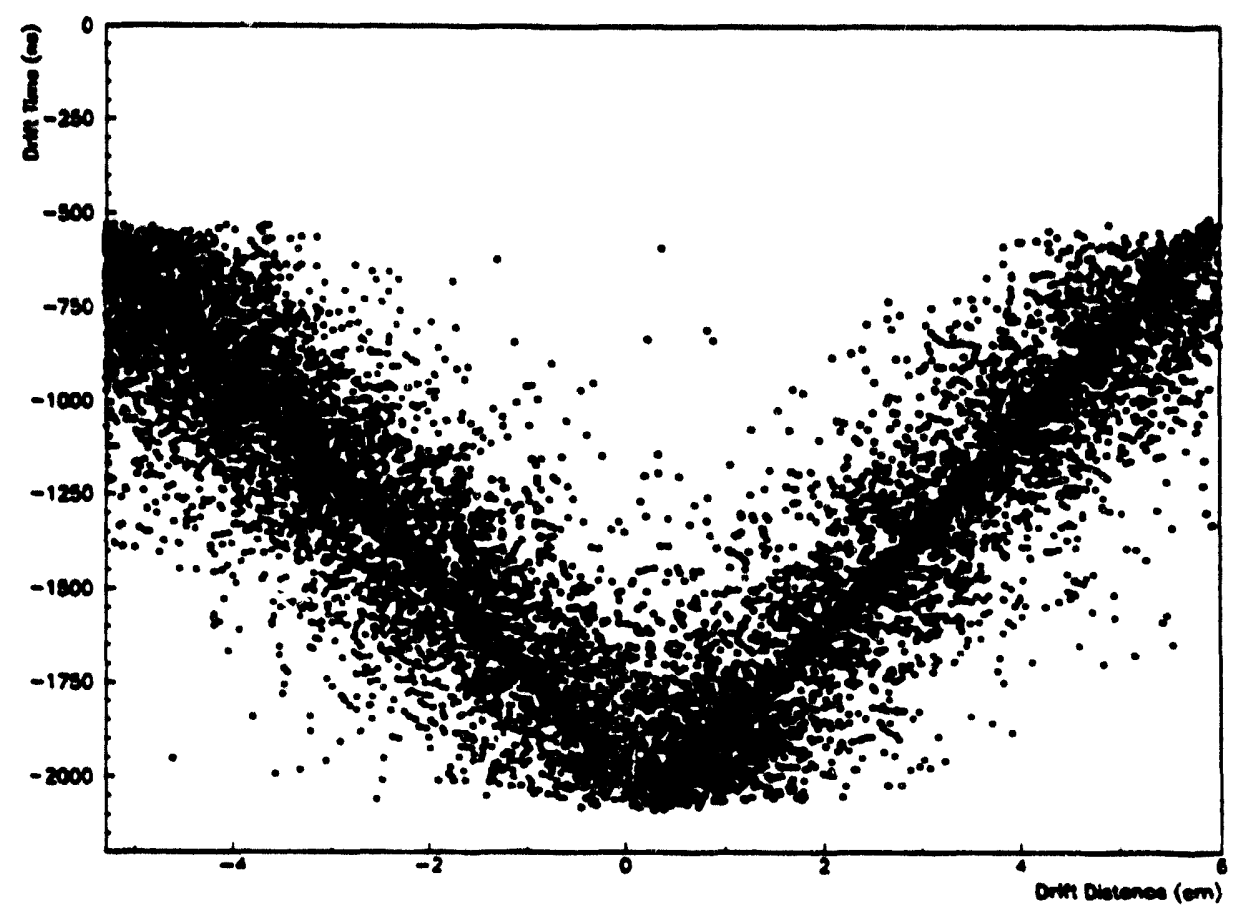

Figure 5.3: Scatter plot of drift time versus PWC drift coordinate before correction for overall $\mathrm{TO}$ and sense wire position.

calibration parameters. The calibration program also determined appropriate hardware zero suppression parameters according to the individual channel's mean baseline and baseline fluctuation data and dowloaded this information into the FADC zero suppresion chip registers (section 2.2.2) .

\section{Overall Electronic Delay and Sense Wire Staggering}

A complete sweep of the center sector (module) was done with the premod aligned in two different positions with respect to the test beam. The premod was first aligned so that the layer 0 - layer 2 sense wire plane was parallel to the beam. Data was then taken beginning at the layer 0 - layer 2 cathodes. The premod was moved in $.5 \mathrm{~cm}$ steps until the layer 0 , layer 2 drift cells were completely surveyed. This procedure was then repeated with the premod aligned along the layer 1 - layer 3 sense wire plane of the center module. 
Figure 5.3 shows a scatter plot of the premod drift time ( $Y$ axis) versus the PWC coordinate ( $X$ axis) for a single sense wire before adjustment of the sense wire position and the overall electronic $\mathrm{T} 0$. The drift times are negative since the trigger time reference pulse was delayed with respect to the chamber signals. A similiar scatter plot was generated for all 28 sense wires of the center module. The scatter plots were sliced along $Y$ in $1 \mathrm{~mm}$ bands and the mean of each drift time projection was found and plotted versus the drift distance.

Figure 5.4 shows the resulting drift time vs drift distance curve. The vertex of the $\mathrm{V}$ shaped curve is where the test beam passes through the sense wire plane. Fitting a $1 \mathrm{~cm}$ wide band of each arm of the vertex to straight line and finding the coordinates of the intersection point allows one to determine the sense wire position with respect to the PWCs and the minimum drift time (overall T0). Figure 5.5 shows the drift distance vs drift time curve after adjustment of the sense wire position and overall TO.

Averaging over the 28 sense wires gives $\langle T 0\rangle=-2083 n s \pm 4 n s$. The sense wire position with respect to the PWCs gives a direct measurement on the sense wire staggering. Averaging over the sense wires gives a mean staggering of $392 \mu m \pm 5 \mu m$ with an rms fluctuation of $30 \mu m$ which is consistent with the nominal staggering of $400 \mu \mathrm{m}$ and expected geometric tolerance of $20 \mu m$ to $50 \mu m$. 


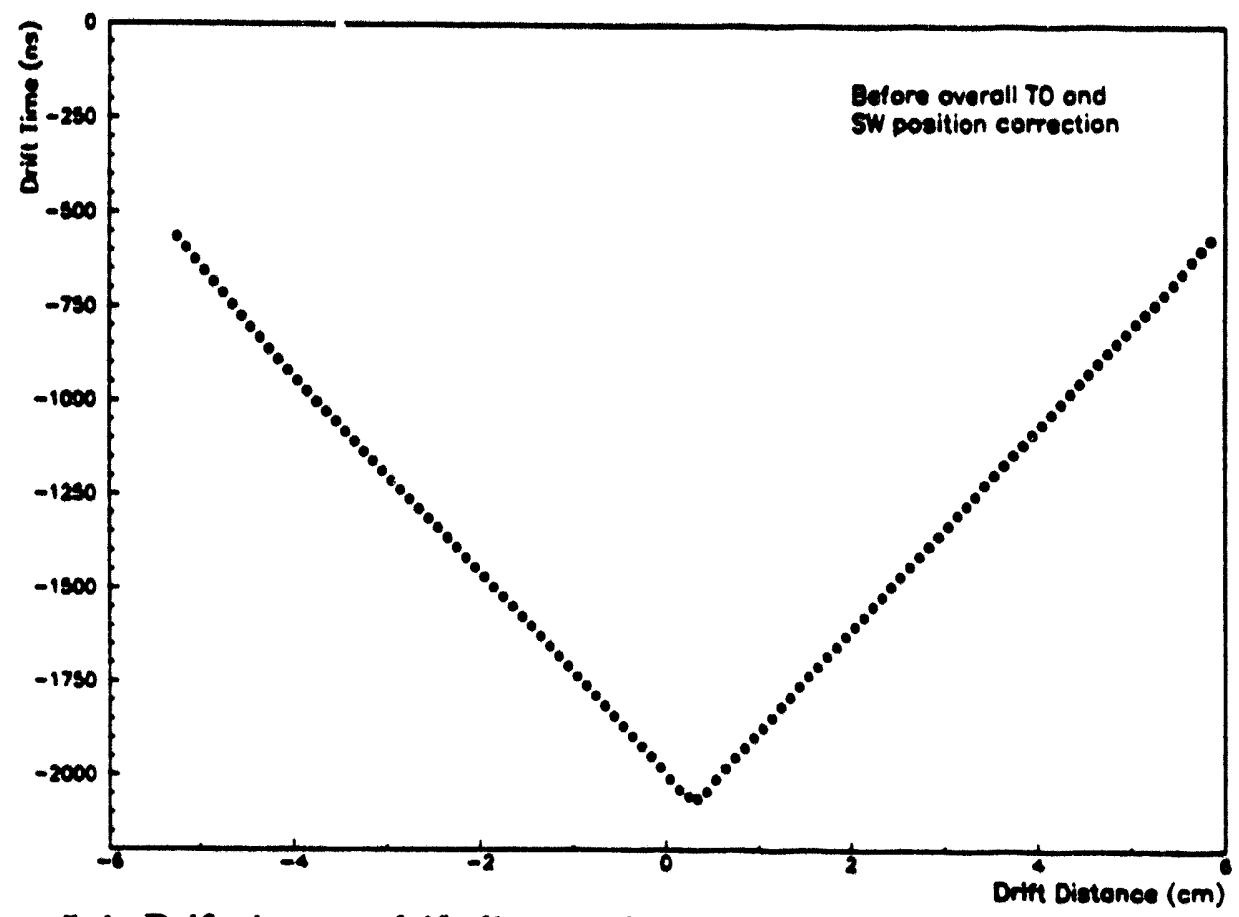

Figure 5.4: Drift time vs. drift distance for a single sense wire before position and overall T0 correction.

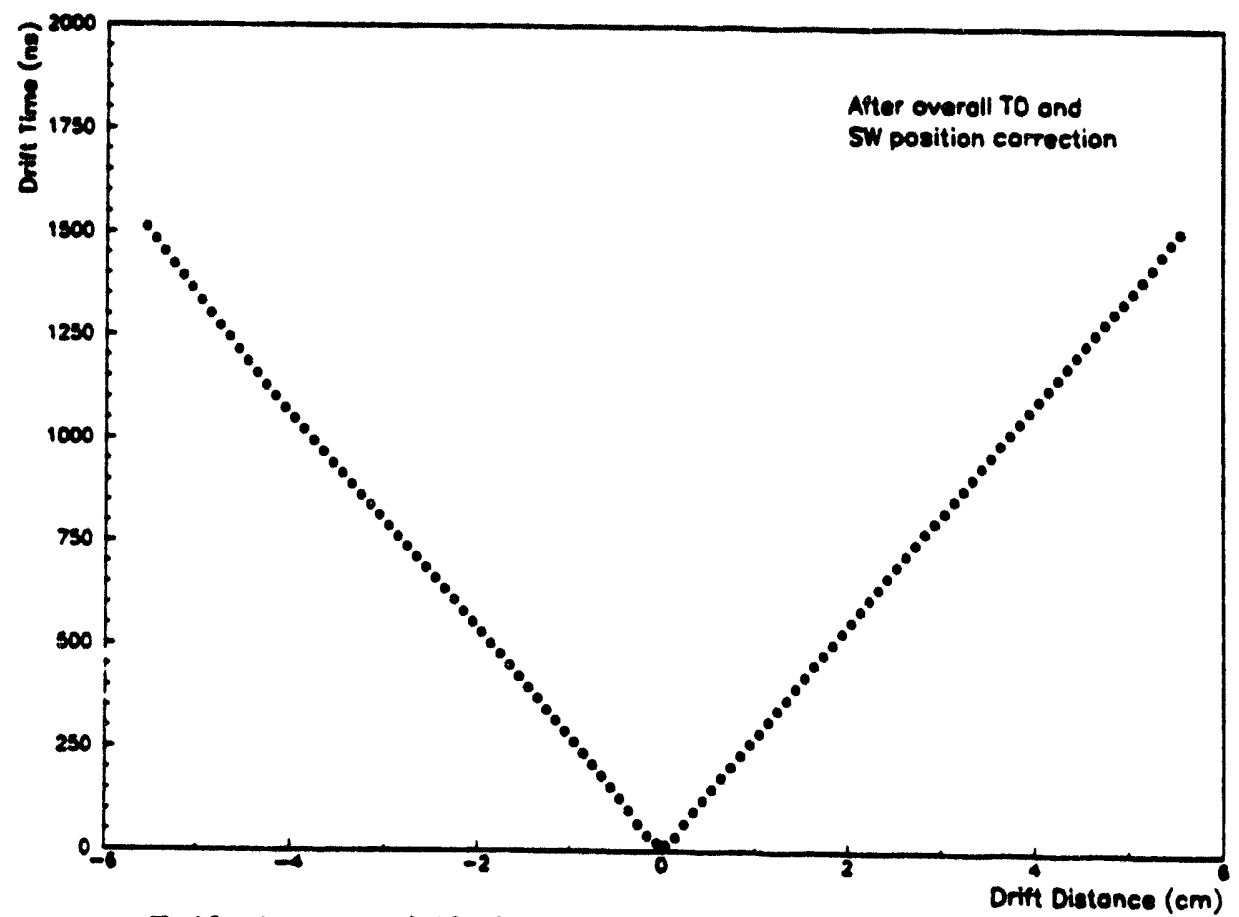

Figure 6.5: Drift time vs. drift distance for a single sense wire after position and overall T0 correction. 


\subsection{Drift Velocity}

All analysis results for the 1989 Stony Brook Cosmic Ray and the Fermilab runs reflect chamber operating voltages of $1460 \mathrm{~V}$ for the inner sense wires, $1590 \mathrm{~V}$ for the outer sense wires, $4200 \mathrm{~V}$ for the cathode, and $450 \mathrm{~V}$ for the central field shaping strip. The gas mix was set at $93 \%$ Argon, $4 \%$ Methane, and 3\% Carbon Dioxide (MARK 2 gas) and was carefully monitored by a computer controlled gas mixing system.

\subsubsection{Fermilab Data}

The addition of PWC tracking information to the Fermilab test beam data allowed a direct measurement of the mean drift velocities as well the drift velocity nonlinearities caused by distortions within the field shaping cage. The event sample was chosen to contain a clean set of 4 segment $R \Phi$ tracks. Each of the 4 track segments was required to have at least 5 of 7 participating sense wire hits and a Chi squared per degree of freedom $\left(\chi^{2} / d f\right)$ less than 5 . A loose road cut of $.15 \mathrm{~cm}$ was used to associate hits to segments. Segments in adjacent layers were associated to a full track by imposing a cut of $.5 \mathrm{~cm}$ on $\Delta D$ and .05 radians on $\Delta \Phi$ (see section 4.2 ).

The mean drift velocity was found for each of the 28 sense wires of the premod's center module by fitting each arm of the adjusted drift time vs. drift distance curve (figure 5.5) to a straight line and constraining the fitted curves to pass through the origin. Figure 5.6 summarizes the mean drift velocities for each layer and for both halves of the drift cell.

Figures 5.8 through 5.11 show the residuals of the fitted curves as a function of absolute drift distance for each of the 28 sense wires in the center module. Both halves of the drift cell are plotted on the same graph with each point representing a $1 \mathrm{~mm}$ wide section of the drift cell. There is strong evidence for systematic nonlinearities which are layer and side dependant as 


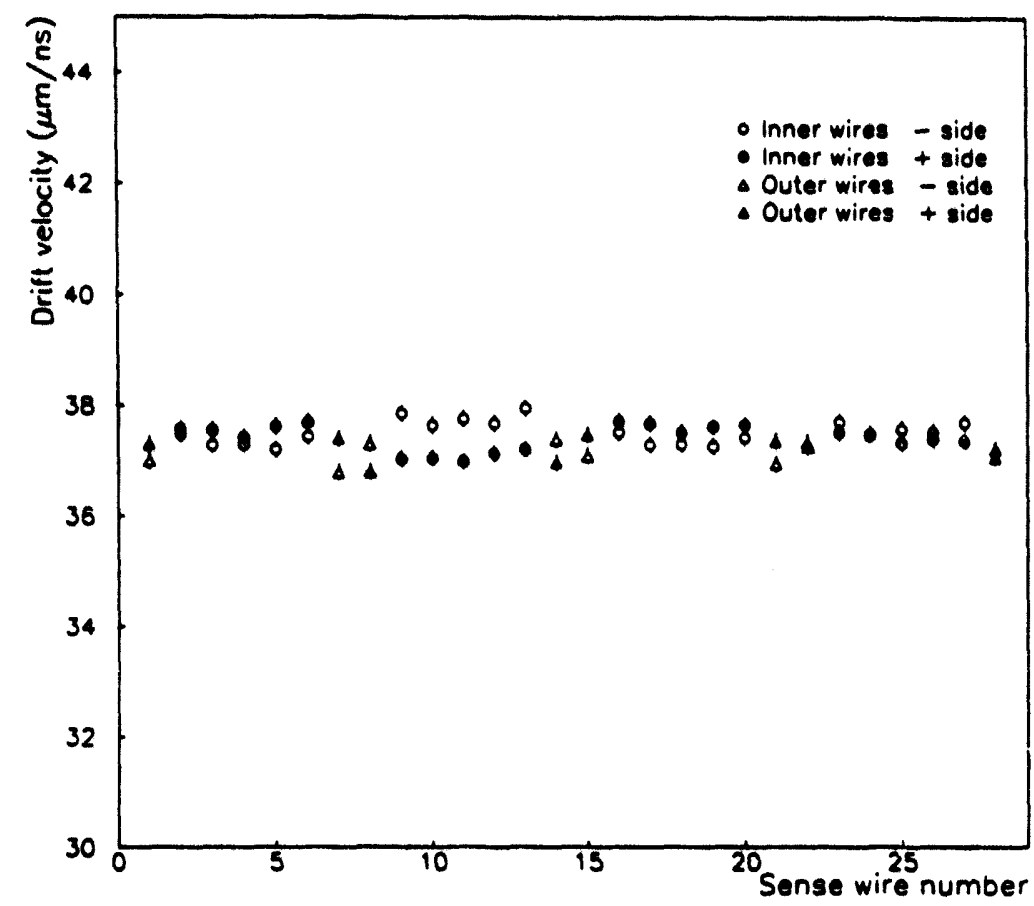

Figure 5.6: Mean drift velocitites as calculated from the Fermilab test data.

well as inner and outer sense wire dependant. Whereas the side dependance is not as evident in the layer 0 drift cell, layers 1 through 3 show a definite dependance of the residual function on the drift side.

Layers 1 and 3 + side field shaping is more uniform than the - side as suggested by the generally flatter residual shape. This effect is reversed for layer 2 with now the - side residual function flatter than that of the + side. All layers exhibit a sharp difference in drift field behavior between the inner and outer sense wires. The outer sense wire residuals have a sinusoidal like shape (wires 0 and 6 ) whereas the inner wires tend towards a parabolic like shape. The field beginning from about 1 to $1.5 \mathrm{~cm}$ from the cathode (large drift distances) shows a systematically large distortion. This effect becomes more pronounced as the width of the drift cell increases in moving from layer 0 to layer 3.

Figure 5.12 shows the residual functions seperately averaged over inner 


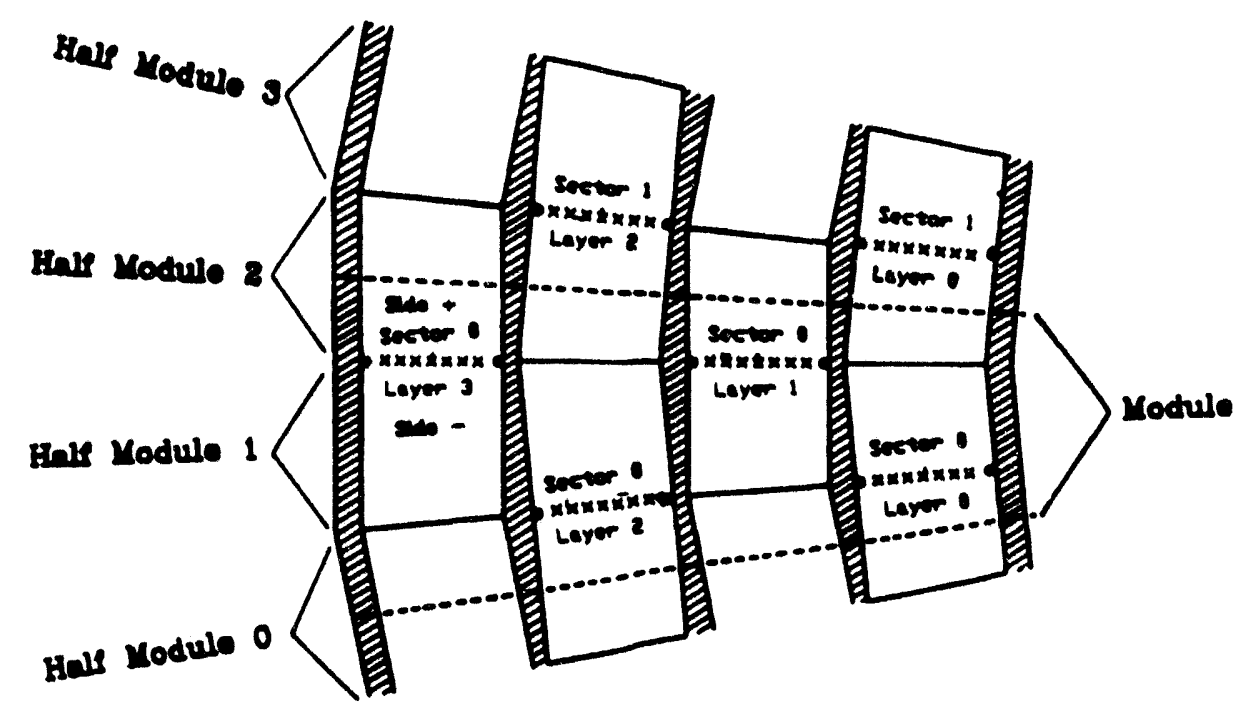

Figure 5.7: $R \Phi$ crossection of part of the central drift chamber showing the relationship between drift cells and half modules.

and outer sense wires for each layer side. Superimposed on each plot are fits to a $5^{\text {th }}$ degree polynomial for the outer wires and a $3^{\text {rd }}$ degree polynomial for the inner wires with the constant term fixed at zero for each fit.

The results suggest 4 residual function shapes: a layer 0,2 - side, layer 1,3 + side inner and outer wire correction and a layer 1,3 - side, layer $0,2+$ side inner and outer wire correction. From figure 5.7 we see that this corresponds to asstematic difference in the drift field configuration between even and odd half modules. The side dependance of the mean drift velocity for inner and outer wires is reflected in figure 5.6. Figure 5.13 shows the four resulting average residual functions after scaling the layer 0,1 , and 2 results up to the layer 3 drift cell width. Table 5.1 summarizes the four residual functions' coefficients. 

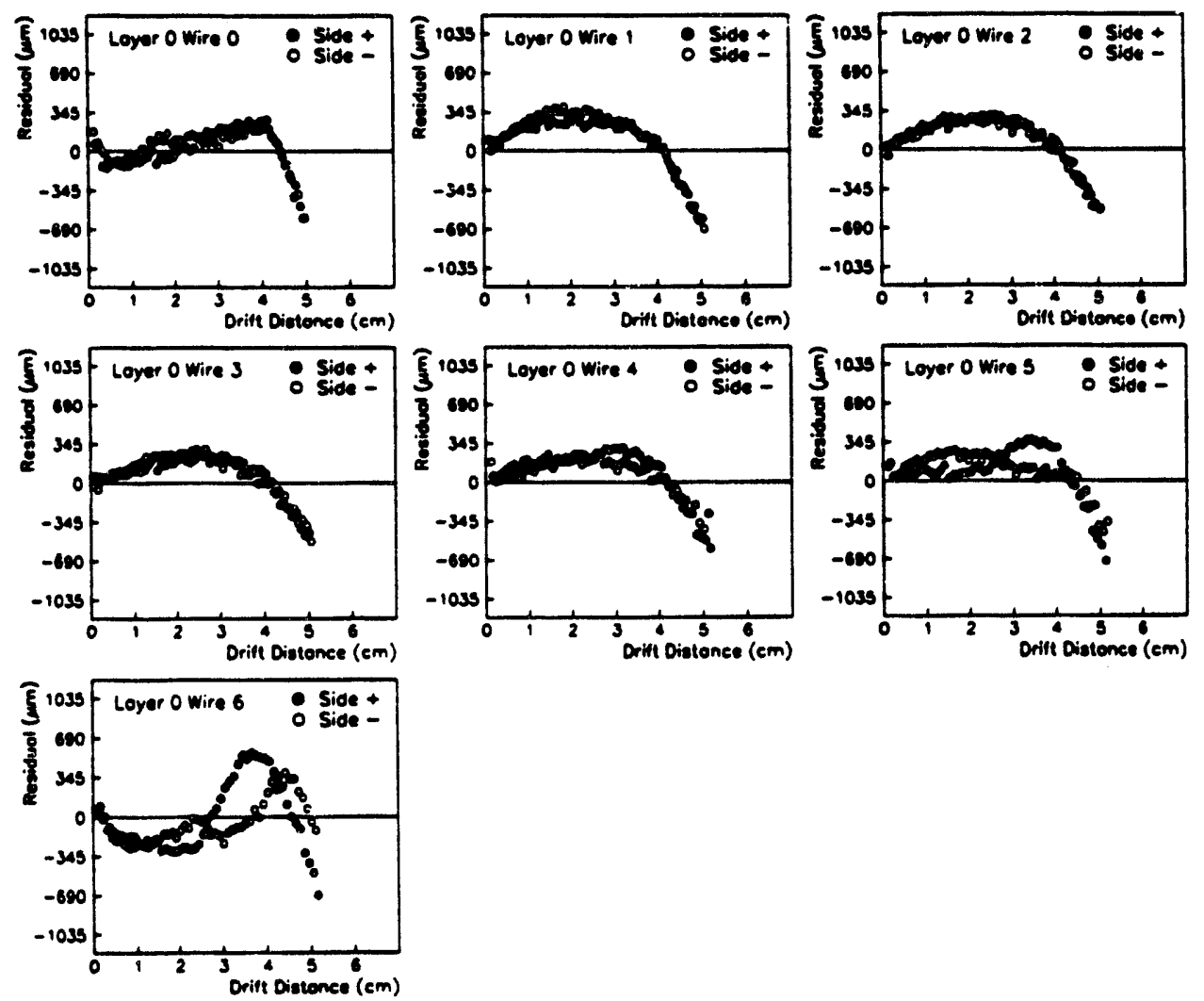

Figure 5.8: Residual vs drift distance functions for sense wires in layer 0 . 

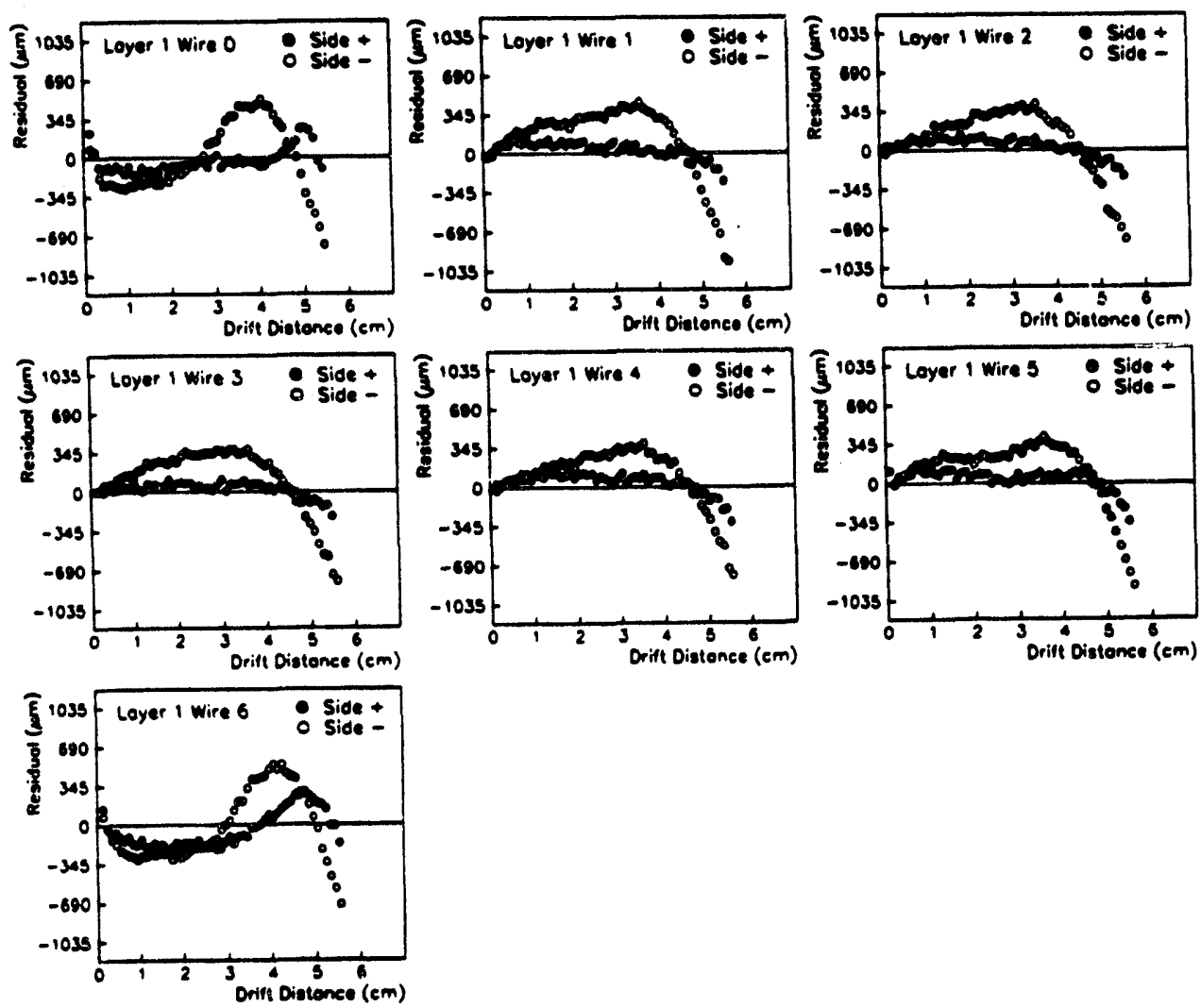

Figure 5.8: Residual vs drift distance functions for sense wires in layer 1 . 

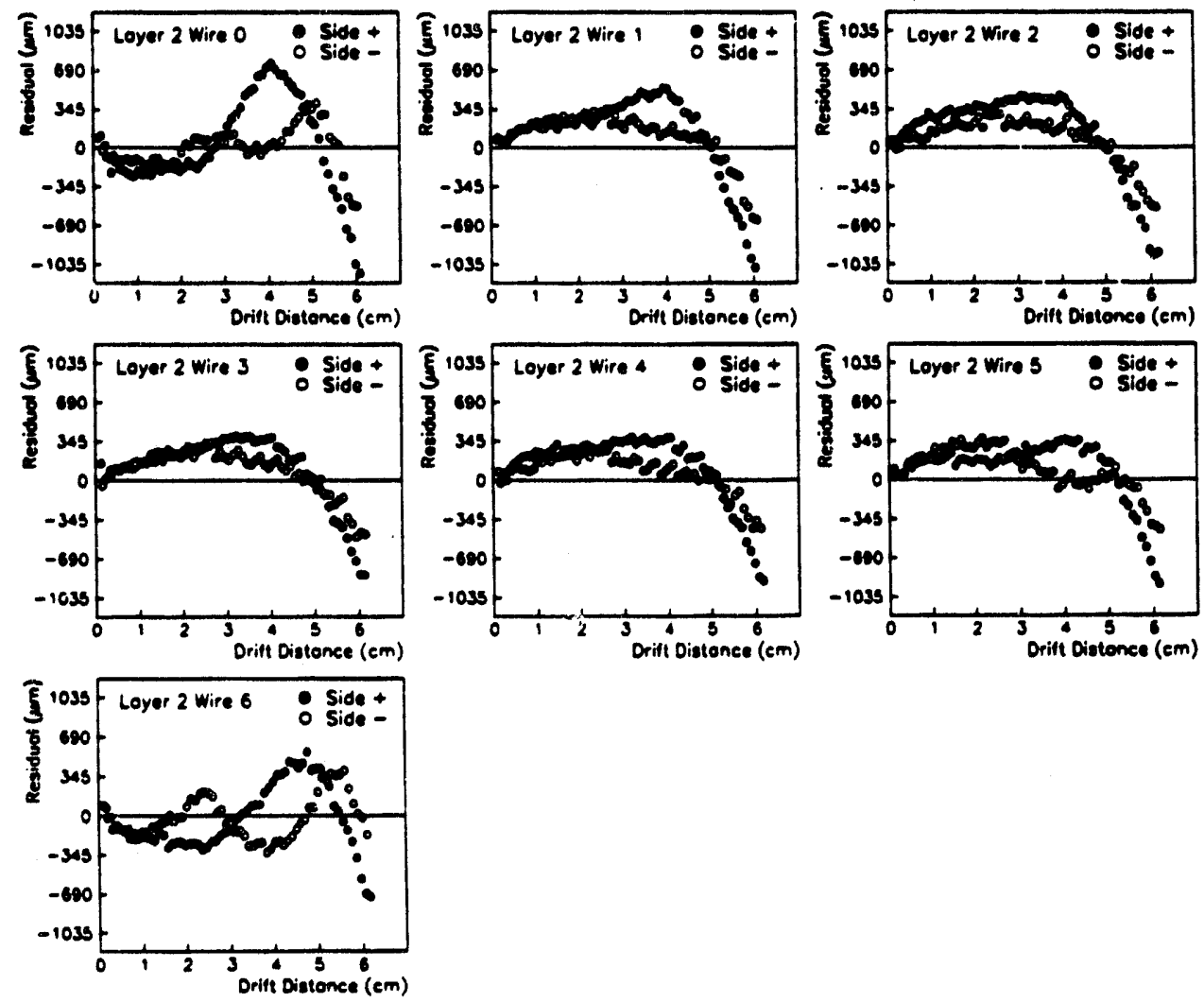

Figure 5.10: Residual vs drift distance functions for sense wires in layer 2. 

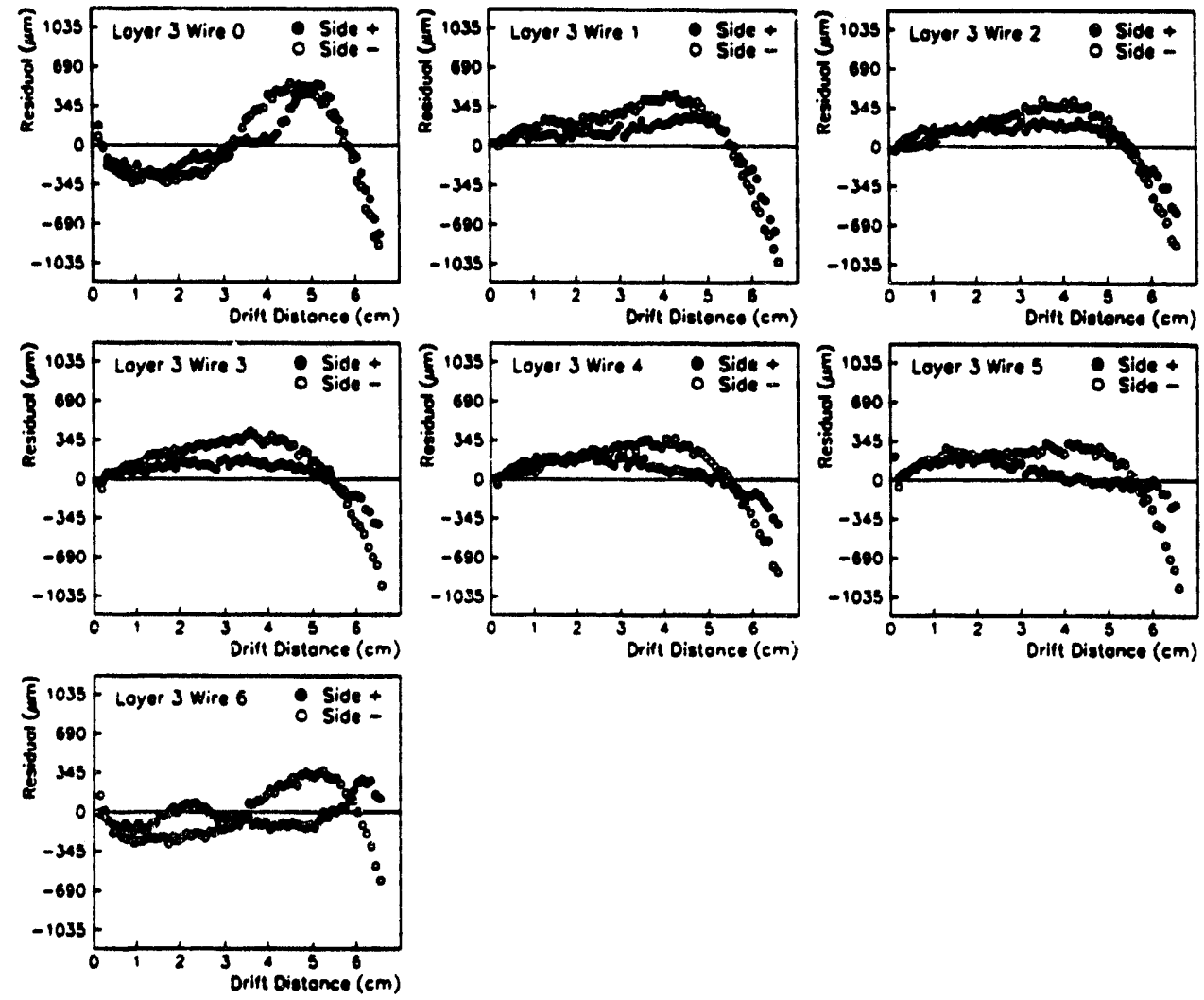

Figure 5.11: Residual vs drift distance functions for sense wires in layer 3. 

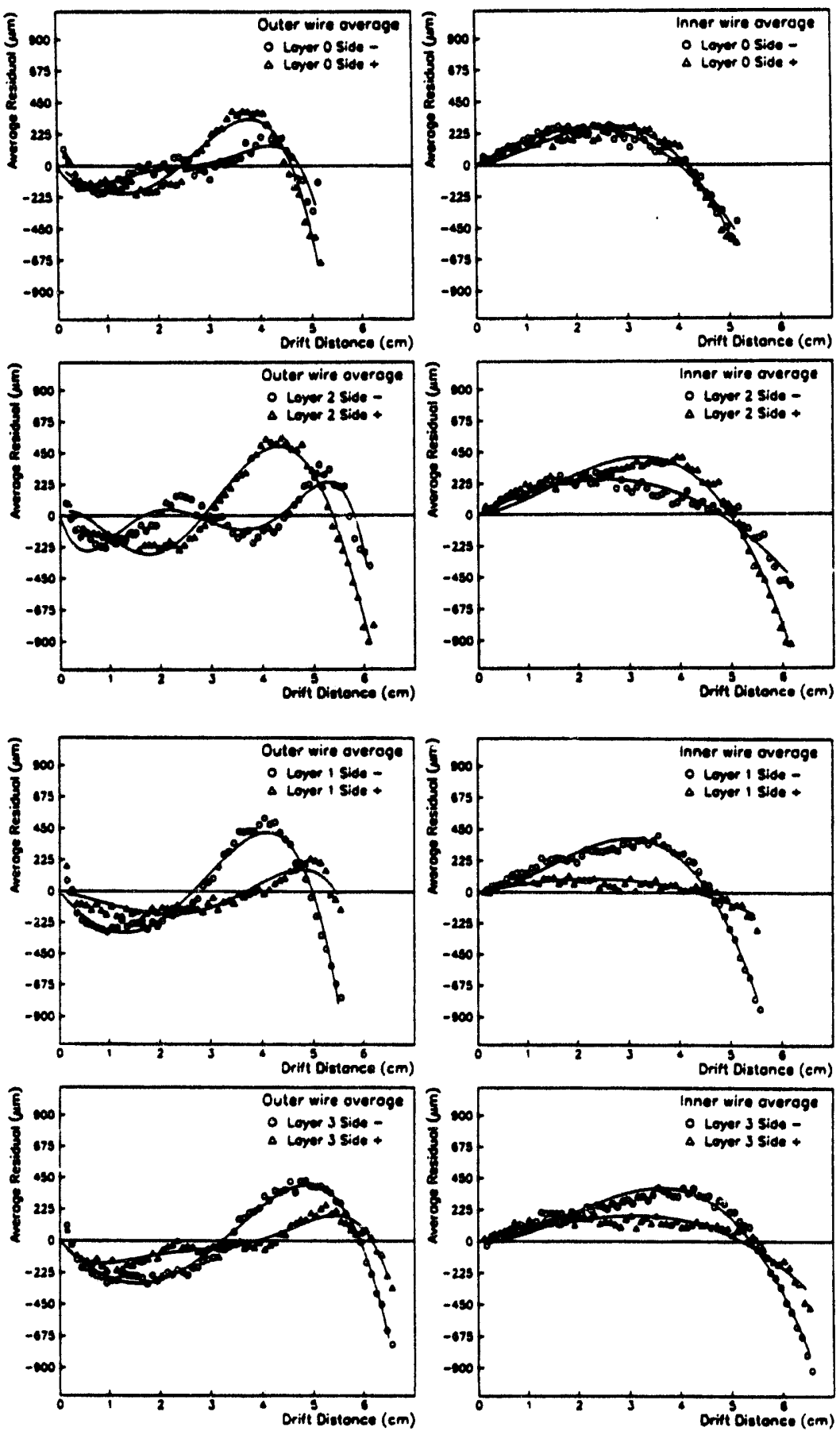

Figure 5.12: Averaged residual vs drift distance functions. 

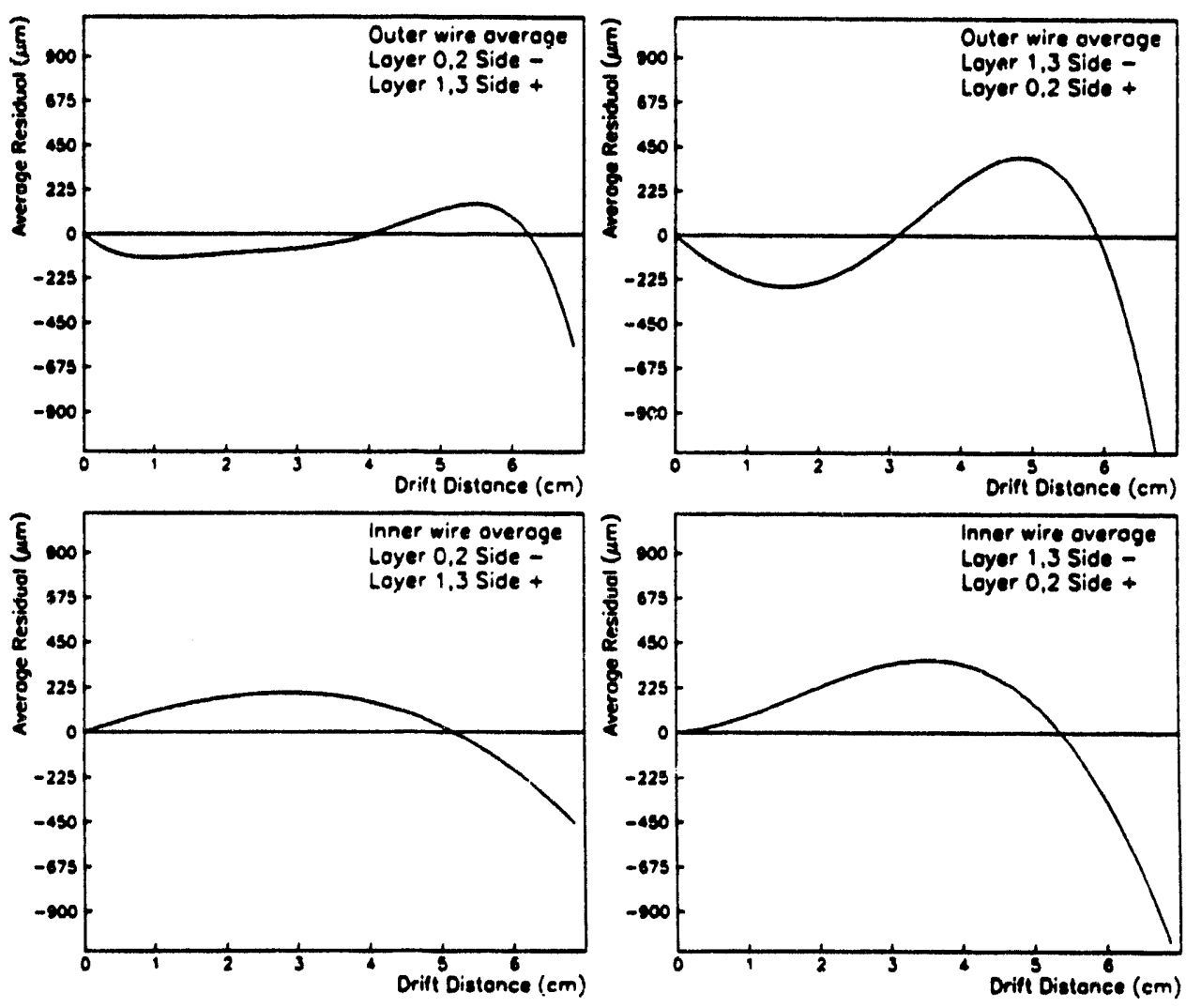

Figure 5.13: The four residual vs drift distance shapes (correction functions) which characterize the central tracking chamber. The function coefficients are summarized in table 5.1 . 


\begin{tabular}{||c|c|c|c|c|c|c|c||}
\hline \hline \multicolumn{8}{|c||}{ Residual Function Coefficients } \\
\hline \hline Layer & Wires & Side & $C_{1}$ & $C_{2}$ & $C_{3}$ & $C_{4}$ & $C_{5}$ \\
\hline 0,2 & Outer & - & -328.354 & 313.024 & -129.808 & 24.953 & -1.738 \\
1,3 & $n$ & + & $n$ & $n$ & $n$ & $"$ & $"$ \\
0,2 & Inner & - & 118.362 & -10.303 & -2.428 & - & - \\
1,3 & $"$ & + & $n$ & $n$ & $n$ & - & - \\
0,2 & Outer & + & -312.521 & 68.165 & 20.335 & -2.503 & -0.232 \\
1,3 & $n$ & - & $"$ & $n$ & $n$ & $"$ & $"$ \\
0,2 & Inner & + & 25.368 & 72.802 & -14.403 & - & - \\
1,3 & $"$ & - & $"$ & $n$ & $n$ & - & - \\
\hline \hline
\end{tabular}

Table 5.1 Coefficients of residual functions. 


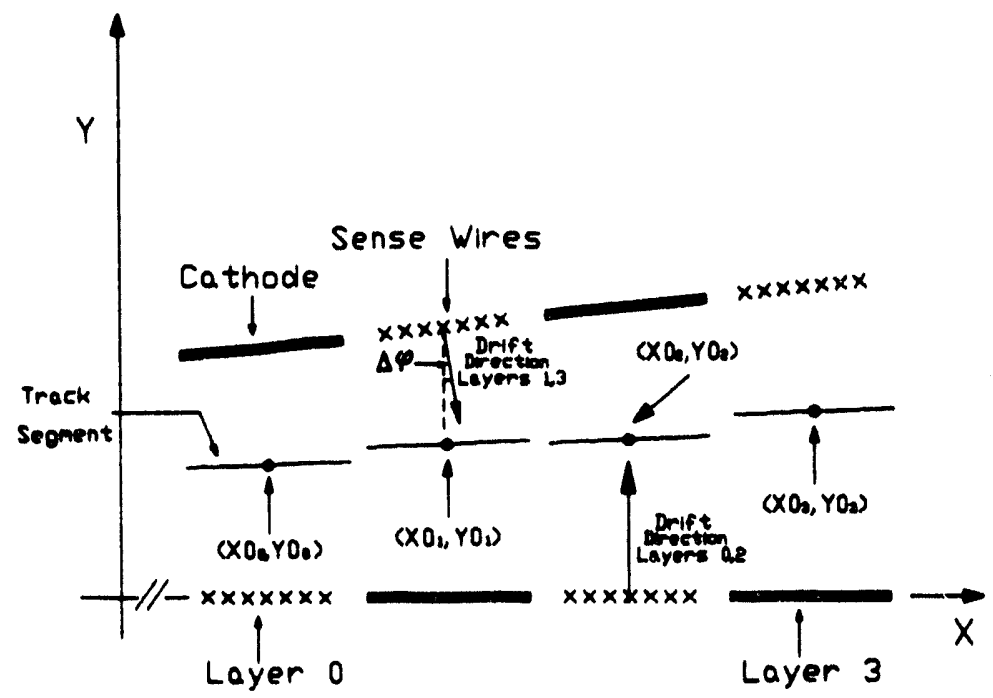

Figure 5.14: A half module of the central tracking chamber in a coordinate system in which the $X$ axis is aligned along the layer 0 - layer 2 sense wire plane.

\subsubsection{Cosmic Ray Data}

To determine the drift velocity for a half module of the D0 central tracking chamber, a clean sample of cosmic ray events containing a low number of spurious hits and a single 4 segment $R \Phi$ track fully contained within a half module were chosen. Cuts on tracking variables were the same as those for the Fermilab data (section 5.5.1).

Since there was no external beam position measurement in this data sample, the drift velocity was found based on three simplifying assumptions:

1. The drift velocity is the same for inner sense wires within the same layer of a half module (figures 5.7 and 5.14 ).

2. The drift velocities for inner sense wires across the 4 layers $(i=0,1,2,3)$ 
of a half module are related by:

$$
V_{i}=\langle V\rangle+\left(\frac{3}{2}-i\right) * \Delta V
$$

with $\langle V\rangle$ and $\Delta V$ the mean velocity and velocity step respectively for a particular half module.

3. The same drift velocity relationship holds for the outer sense wires, however, $\langle V\rangle$ and $\Delta V$ need not be the same as for the inner wires within the half module.

A charged particle traversing the Do central tracking chamber will, in most cases, generate a track segment in each of the 4 layers. Considering only tracks which are contained within a half module, the midpoint of each segment $Y O_{i}$ in a coordinate frarne in which the $X$ axis is along the layer 0 - layer 2 sense wire plane is according to assumptions 1 and 2 above given by

$$
\begin{aligned}
Y 0_{0} & =\left(V+\frac{3}{2} \Delta V\right) T_{0} \\
Y 0_{1} & =\delta_{1}-\left(V+\frac{1}{2} \Delta V\right) T_{1} / \cos (\Delta \phi) \\
Y 0_{2} & =\left(V-\frac{1}{2} \Delta V\right) T_{2} \\
Y 0_{3} & =\delta_{3}-\left(V-\frac{3}{2} \Delta V\right) T_{3} / \cos (\Delta \phi)
\end{aligned}
$$

where $T_{i}$ is taken to be the average electron arrival time as measured from the sense wires participating in the segment for the $i$ th layer, $\Delta \phi$ is the relative drift angle of layers 1 and 3 with respect to layers 0 and 2 , and $\delta_{i}=X 0_{i} \tan \Delta \Phi$. Referring to figure 5.14, we can impose two alignment constraints by forcing that the partial track formed by the layer 0 and layer 2 segments lie on top of the partial track formed by the layer 1 and layer 3 segments. This can be expressed by the conditions

$$
\begin{aligned}
& Y 0_{0}-\left[Y 0_{13}-M_{13}\left(X 0_{13}-X 0_{0}\right)\right]=0 \\
& Y 0_{2}-\left[Y 0_{13}-M_{13}\left(X 0_{13}-X 0_{2}\right)\right]=0
\end{aligned}
$$


where

$$
\begin{aligned}
Y 0_{13} & =\frac{Y 0_{1}+Y 0_{3}}{2} \\
M_{13} & =\frac{Y 0_{3}-Y 0_{1}}{X 0_{3}-X 0_{1}} \\
X 0_{13} & =\frac{X 0_{1}+X 0_{3}}{2}
\end{aligned}
$$

Substituting 5.8 thru 5.10 into 5.6 and 5.7 gives

$$
\begin{aligned}
& Y 0_{0}\left(X 0_{3}-X 0_{1}\right)-\left(Y 0_{3}-Y 0_{1}\right) X 0_{0}=Y 0_{1} X 0_{3}-Y 0_{3} X 0_{1} \\
& Y 0_{2}\left(X 0_{3}-X 0_{1}\right)-\left(Y 0_{3}-Y 0_{1}\right) X 0_{2}=Y 0_{1} X 0_{3}-Y 0_{3} X 0_{1}
\end{aligned}
$$

Defining $\beta_{i j}=X 0_{i}-X 0_{j}$ and substituting equations 5.2 thru 5.5 into 5.11 and 5.12 , we arrive at a set of simultaneous equations for $V$ and $\Delta V$

$$
\begin{aligned}
& \alpha V+\gamma \Delta V=\zeta \\
& \eta V+\lambda \Delta V=\xi
\end{aligned}
$$

whose solution is

$$
\begin{aligned}
V & =\frac{\zeta \lambda-\gamma \xi}{\lambda \alpha-\eta \gamma} \\
\Delta V & =\frac{\xi \alpha-\eta \zeta}{\lambda \alpha-\eta \gamma}
\end{aligned}
$$

with

$$
\begin{aligned}
\alpha & =T_{0} \beta_{31}+T_{1} \beta_{01} / \cos \Delta \phi \\
\gamma & =\frac{3}{2} T_{0} \beta_{31}+\left(\frac{1}{2} T_{1} \beta_{30}+\frac{3}{2} T_{3} \beta_{10}\right) / \cos \Delta \phi \\
\eta & =T_{2} \beta_{31}+\left(T_{1} \beta_{32}+T_{3} \beta_{21}\right) / \cos \Delta \phi \\
\lambda & =-\frac{1}{2} T_{2} \beta_{31}+\left(\frac{1}{2} T_{1} \beta 32+\frac{3}{2} T_{3} \beta_{12}\right) / \cos \Delta \phi \\
\zeta & =\delta_{1} \beta_{30}+\delta_{3} \beta_{01} \\
\xi & =\delta_{1} \beta_{32}+\delta_{3} \beta_{21}
\end{aligned}
$$



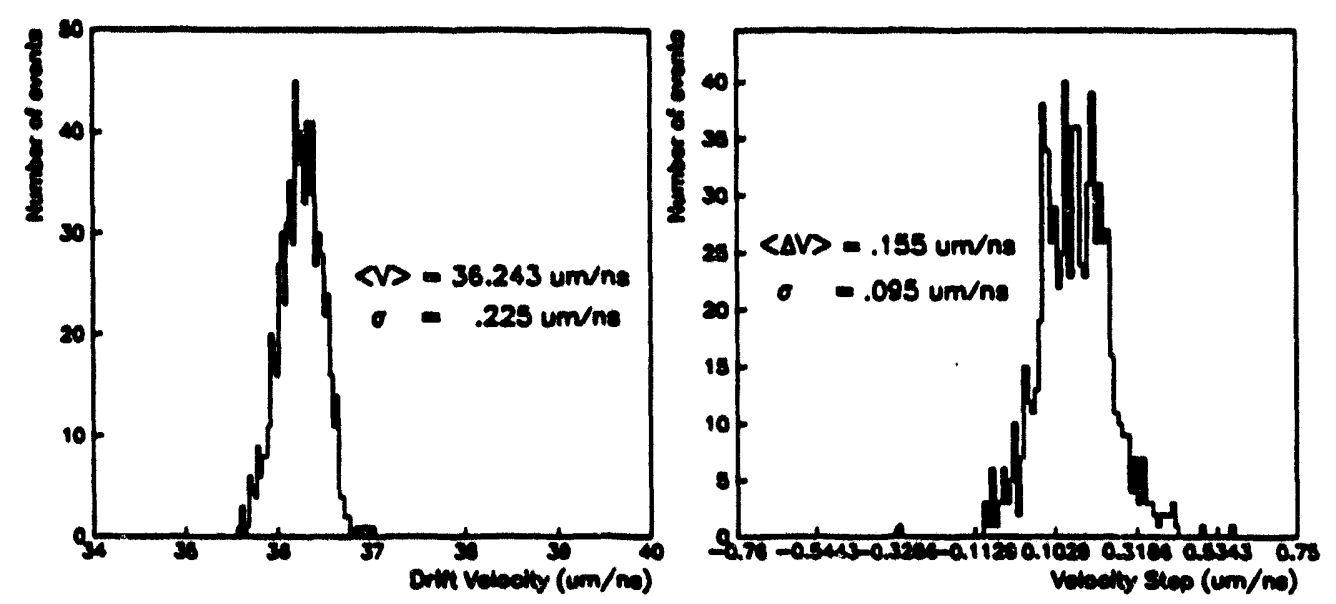

Figure 5.15: Drift velocity and velocity step distribution for a typical $1 / 2$ module of the CDC.

For each cosmic ray event containing a 4 segment track, the velocity and velocity step were determined from equations 5.13 and 5.14 . Figure 5.15 shows the velocity and velocity step distributions for a typical half module of the chamber. The narrow widths of the mean velocity and velocity step distributions allow a precise drift velocity measurement with extremely low statistics. A sample of only 10 tracks gives a statistical uncertainty of less than $.15 \%$ on the mean drift velocity across the four layers of the half module. The systematic error on the mean velocity depends on how well the electronic delays are known. The uncertainty in the mean velocity $\langle V\rangle$ for a lns uncertainty in the overall time delay $T 0$ is measured to be

$$
\frac{d<V\rangle}{d T 0}=.0478 \mu \mathrm{m} / \mathrm{ns}^{2}
$$




\subsection{Sense Wire Efflciencies}

The sense wire efficiencies were calculated by determining the ratio between the number of times that a sense wire participates in a 4 segment $R \Phi$ track and the total number of 4 segment tracks in the event sample. Tracks which crossed either the sense wire or cathode planes of a cell were excluded from the analysis. Figures 5.16 and 5.17 show the efficiencies for both inner and outer sense wires respectively with each plot containing wire data broken down on a per layer basis. Each inner sense wire plot represents 160 wires (5 inner wires/cell $\times 32$ cells/layer) while each outer sense wire plot represents 64 wires ( 2 outer wires/cell $\times 32$ cells/layer). Table 5.2 summarizes the average efficiencies and their rms fluctuation for each layer and for both inner and outer sense wire groups. The results are consistent with the measured overall wire efficiency of $94 \pm 2 \%$ by Behnke. ${ }^{11}$

\begin{tabular}{|c|c|c|}
\hline Layer & \multicolumn{2}{|c|}{ Mean Efficiency } \\
\hline \multirow{3}{*}{0} & Inner Wires & Outer Wires \\
\cline { 2 - 3 } 1 & $97.4 \pm 1.3 \%$ & $93.4 \pm 3.2 \%$ \\
2 & $97.1 \pm 2.4 \%$ & $93.8 \pm 2.5 \%$ \\
3 & $97.7 \pm 1.2 \%$ & $92.7 \pm 3.0 \%$ \\
\hline$\langle E f f\rangle$ & $96.8 \pm 3.0 \%$ & $93.9 \pm 3.2 \%$ \\
\hline \hline
\end{tabular}

Table 5.2 Summary of sense wire efficiencies. 

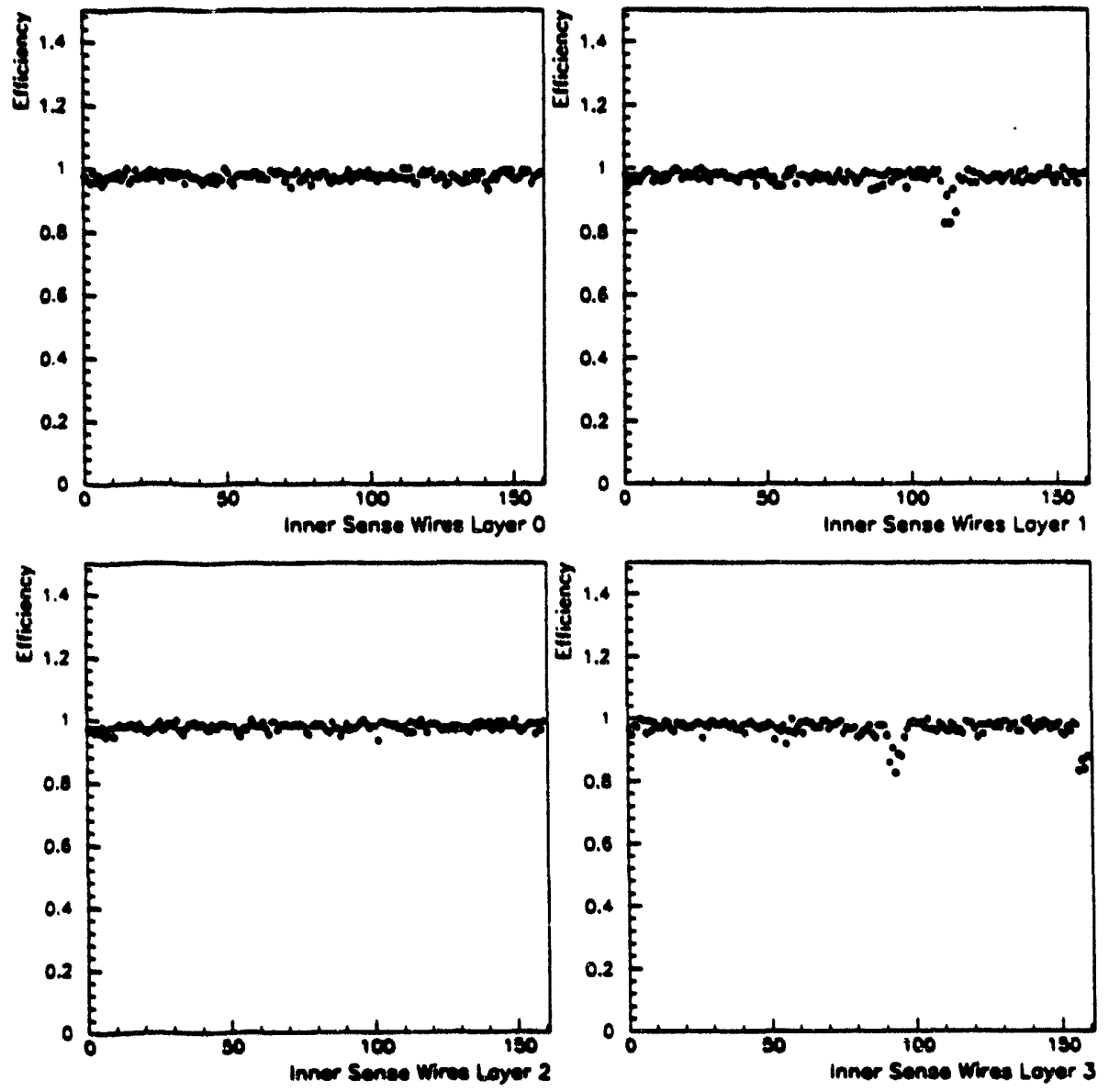

Figure 5.16: Inner sense wire efficiencies. 

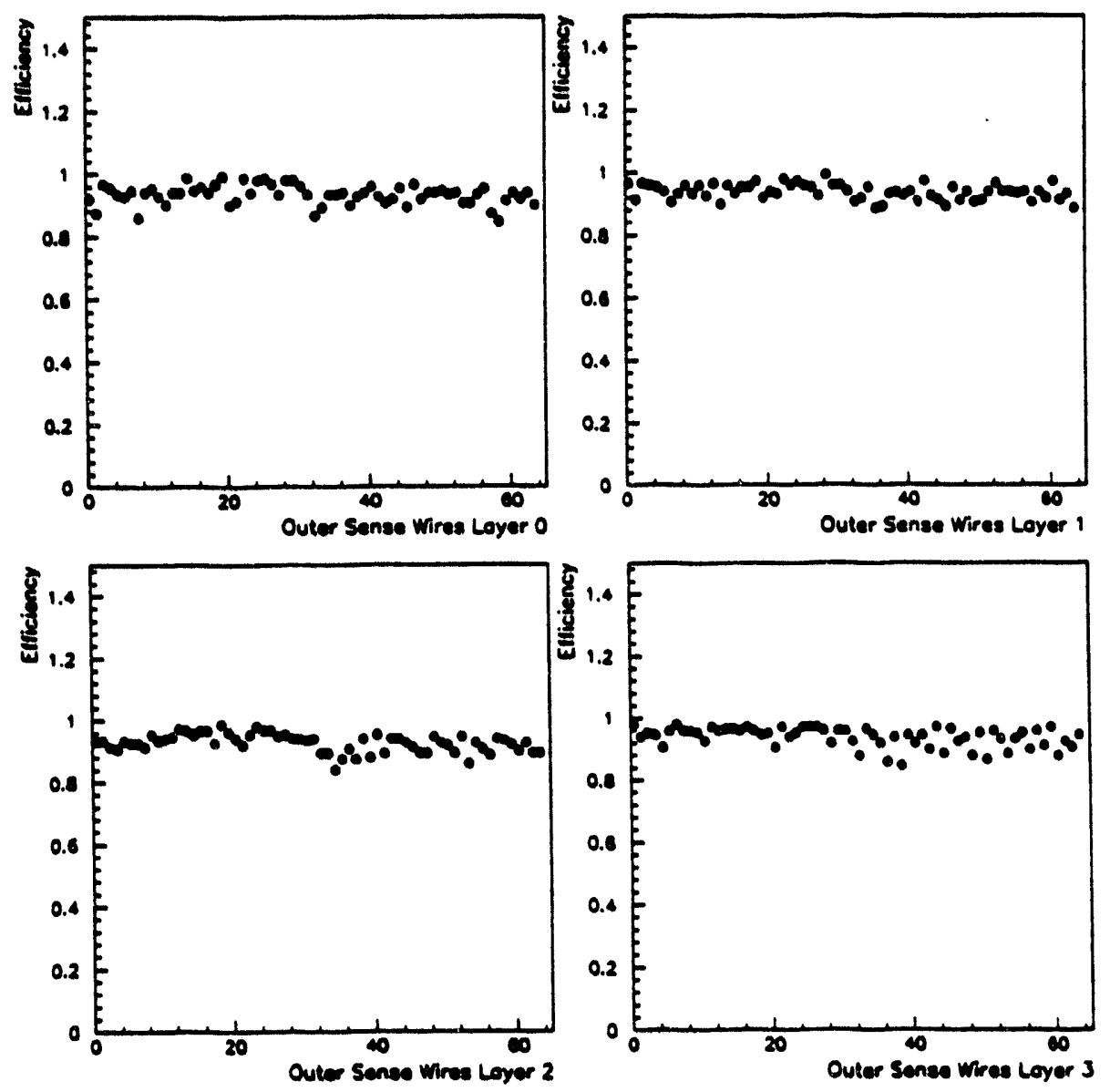

Figure 5.17: Outer sense wire efficiencies. 


\subsection{Sense Wire Resolution}

The accuracy with which an $R \Phi$ track can be reconstructed depends principally on a detailed knowledge of the chamber geometry, the drift time to space relationship (drift velocity), and the diffusion of the drifting electron swarm as it approaches the anode wires. ${ }^{21}$ Additional considerations such as electronic noise, individual electronic channel delays, pulse shaping, and software hitfinding optimization also contribute to the reconstruction accuracy. In general, however, the contribution from these sources can be kept small by proper calibration and monitoring in comparison to contributions from electron diffusion and non-uniformities in the drift field.

The $R \Phi$ resolution as a function of drift distance for a drift cell of the Do central tracking chamber was measured from the cosmic ray and Fermilab test beam events by selecting a sample of clean tracks containing at least 3 out of 4 segments. Each ent was required to have a minimum of 5 out of 7 participeting wires $2 / d f$ less than 5 . The sense wire residuals were found by fitting only inner sense wires on a subsample of segments which contained hits from all 5 inner sense wires. The drift cell resolution is then given by the width of the residual distribution Since the inner wires were contained in the fit, the measured inner wire resolution was scaled by a factor of 1.3 as determined from Monte Carlo to compensate for the systematics of the fitting process.

The outer sense wire resolution was determined by measuring the difference between the outer wire hit and an extrapolation of the inner wire fit. The width of the residual distribution in this case does not give a direct measurement of the outer wire resolution since the extrapolation accuracy depends on the resolution of the inner wires. A Monte Carlo study showed that for the given chamber geometry, a good approxination to the intrinsic outer wire 
resolution $\sigma_{0}$ is given by

$$
\sigma_{0} \approx \sqrt{\sigma_{m}^{2}-\sigma_{i}^{2}}
$$

where $\sigma_{m}$ is the measured outer wire resolution and $\sigma_{i}$ is the intrinsic inner wire resolution.

Figue 5.18 shows the sense wire resolution versus drift distance for both inner and outer sense wires averaged over the complete drift chamber (cosmic ray data). Also shown is the sane measurement made with the chamber premod (Fermilab data) using a narrowly collimated test beam oriented parallel to the sense wire plane. The Fermilab Monte Carlo is discussed in sections 5.7.2 and 5.7.3. The difference between the Fermilab and cosmic ray Monte Carlo is due to the additional effects of tracks making a nonzero angle relative to the sense wire plane (section 5.7.4). The Monte Carlo necessarily breaks down at small drift distances due to the complicated nature of the drift field near the anode (section 5.7.1).

The full Fermilab resolution curve is well parameterized by a function of the form

$$
\sigma(X)=A e^{-b X}+\sqrt{c_{1}^{2}+c_{2}^{2} X}
$$

with $A, b$ empirically determined from the Fermilab data for small drift distances $(X<1 \mathrm{~cm})$ and $c_{1}, c_{2}$ calculated from first principles (sections $\mathbf{5 . 7 . 2}$ and 5.7.3). The resolution curve parameters are shown in table 5.3.

\begin{tabular}{||c|c|c|c|c||}
\hline \multicolumn{5}{||c||}{ Sense Wire Resolution vs. Drift Distance } \\
\hline \hline Wire Group & $A(\mu m)$ & $b\left(\mathrm{~cm}^{-1}\right)$ & $c_{1}(\mu m)$ & $c_{2}(\mu m / \sqrt{c m})$ \\
\hline Inner & 500 & -5.3 & 54 & 74 \\
Outer & 500 & -3.3 & 66 & 67 \\
\hline \hline
\end{tabular}

Table 5.3 Sense wire resolution vs. drift distance function coefficients. 

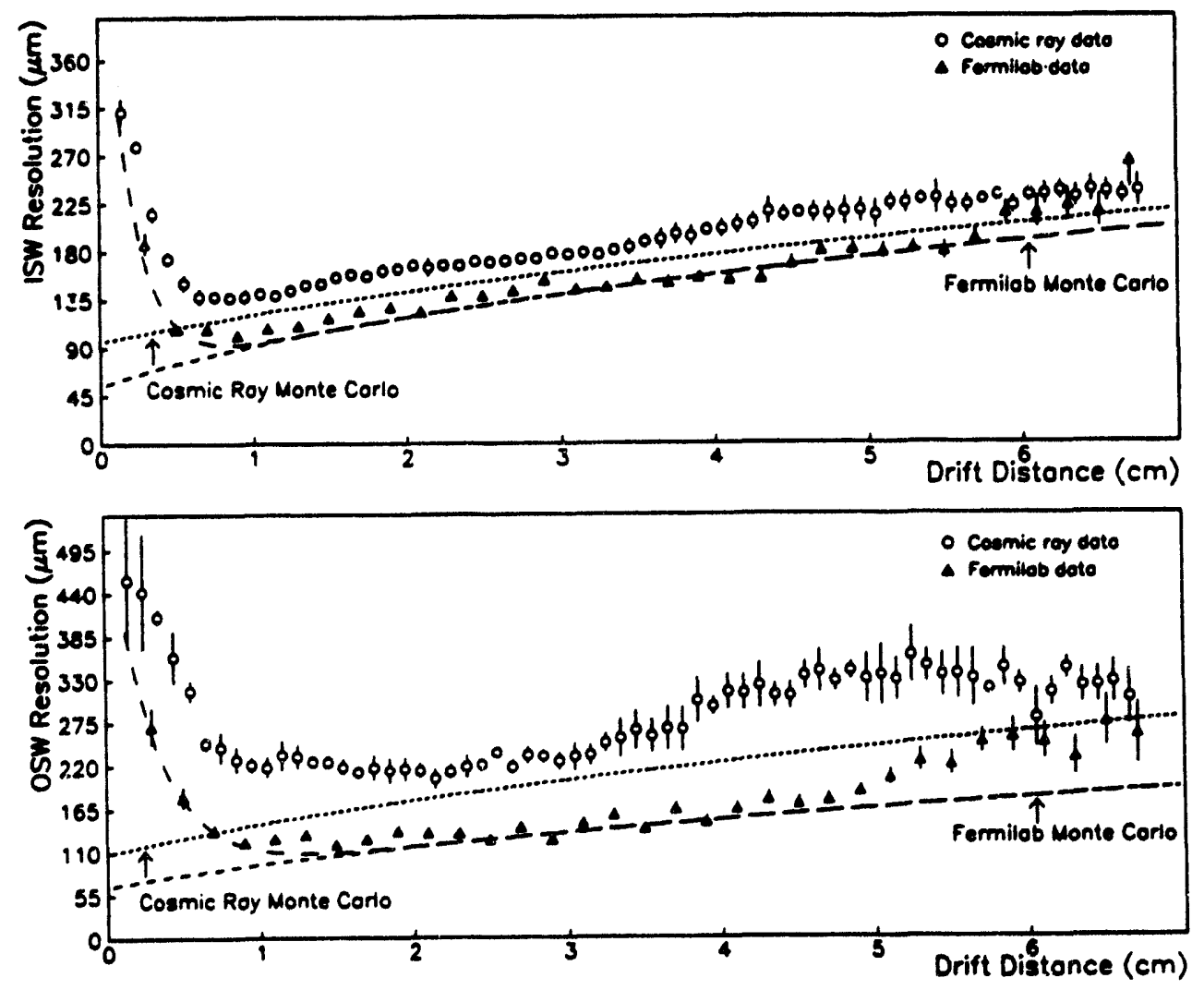

Figure 5.18: Inner and outer sense wire resolution vs. drift distance for the CDC drift cell. 


\subsubsection{Near Anode Region}

The exponential term is dominant for small drift distances $(X \rightarrow 0)$ and parameterizes the complicated effects of primary electron statistics and the highly non linear drift velocity region near the anodes. The average pulse shapes for events in this region is given by the first plot in figure 5.19. The pulses at $X=.5 \mathrm{~mm}$ exhibit a clear two peak structure which the hitfinder may interpret as seperate space hits. In general, this region is further complicated by th uncertainty of which side of the sense wire the ionizing event occurred (section 4.1). Therefore, making an unambiguous association of a hit to a segment is particularly difficult and the near anode tracking resolution is degraded significantly. The addition of external PWC tracking information for the test beam data allowed an effective elimination of the left-right hit ambiguity and an improved near anode resolution when compared to the cosmic ray data.

\subsubsection{Constant Term}

The constant term $c_{1}$ in equation 5.16 is the combined result of electron dispersion arising from the field configuration of the drift cell and drift time measurement errors related to the electronics.

\section{Dispersion}

On average, a minimum ionizing particle traversing an argon filled chamber at standard pressure and temperature will liberate $\approx 30$ electrons per $\mathrm{cm}$ through direct ionization (primary ionization). These primary electrons are energetic enough to liberate $\approx 2$ additional electrons (secondary ionization) per primary electron for a total of $\approx 90$ electrons per $\mathrm{cm}$. The secondary electrons are produced very close to the primary electrons so on average there exists 30 

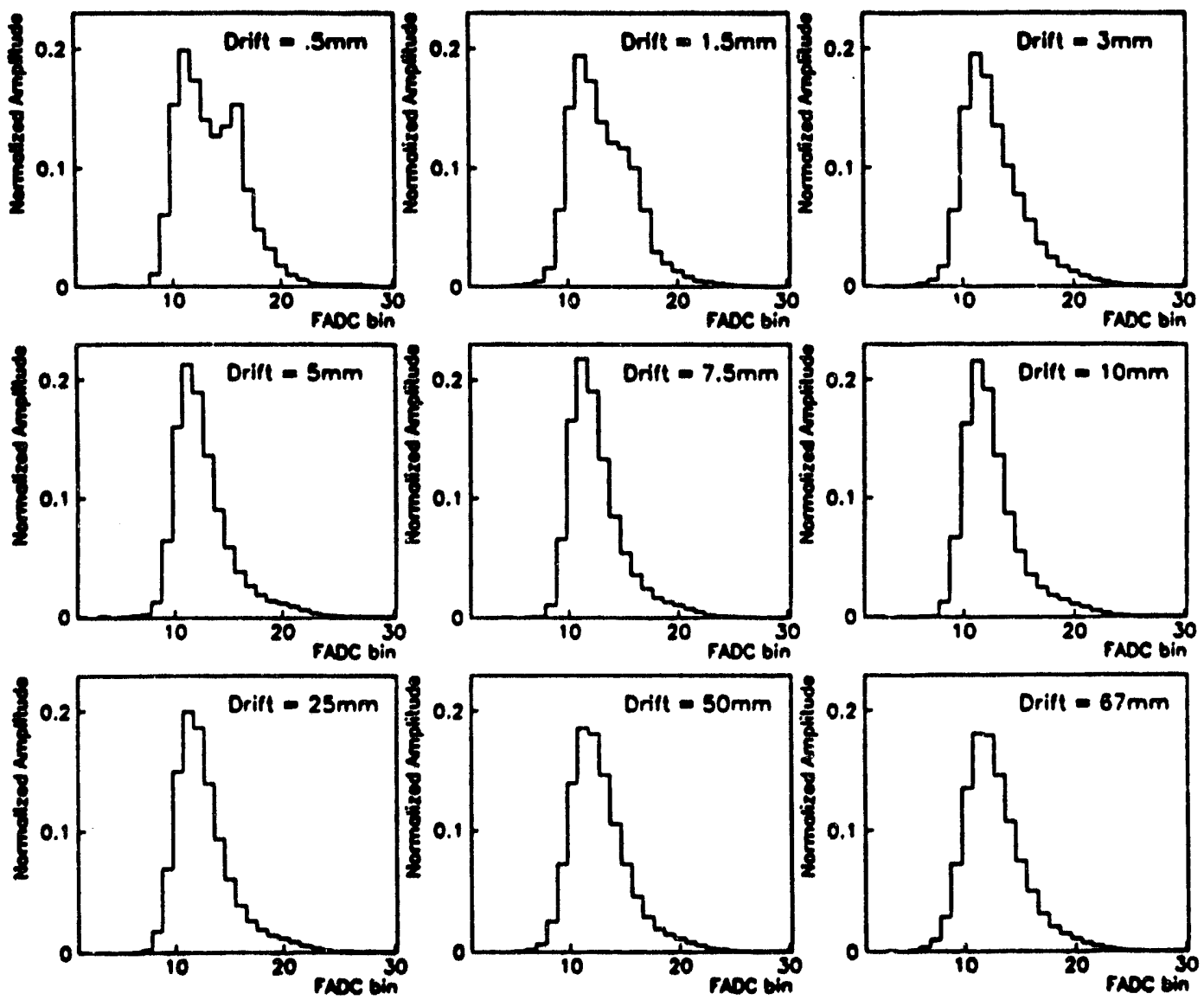

Figure 5.19: Mean sense wire pulse shapes at various drift distances. 
seperate electron groups per $\mathrm{cm}$ along the ionizing track with 3 electrons per group.

For the case of an ionizing track which is parallel to the sense wire plane and for drift in an ideal uniform electric field, all electrons would arrive at the anode simultaneously. In practice however, the actual field shape of the DO drift cell gives rise to a position dependent arrival time for each cluster. Figure 2.6 shows the field configuration for a typical sense wire. The vertical lines are the electric field lines and the horizontal lines show the lines of equal drift time, that is, electrons liberated along one of these lines will arrive at the anode simultaneously. Since an ionizing track can never liberate electrons exclusively on a single equal time line, there always exists some dispersion in the arrival times of the electron clusters particularly as one approches the anode where the field is highly non uniform.

Figure $5.20^{11}$ shows the calculated arrival time of electrons at $3 \mathrm{~cm}$ of drift for inner and outer sense wires versus the position along the gap in which the electrons are liberated for an ionizing track which is parallel to the sense wire plane. The dispersion contribution to the constant term was found by simulating the random liberation of electron clusters in argon by a minimum ionizing particle at a nominal $3 \mathrm{~cm}$ from the anode and parameterizing the arrival time of each cluster depending on its position along the gap according to figure 5.20. For each simulated event, the distribution in mean arrival time for all electrons reaching the wire is shown in figure 5.21. The width of the inner and outer wire distributions gives a dispersion of $\sigma_{d i}=50 \mu \mathrm{m}$ and $\sigma_{d o}=63 \mu \mathrm{m}$ respectively for a nominal drift velocity of $37 \mu \mathrm{m} / \mathrm{ns}$

\section{Electronics}

The analog to digital sampling rate of 106 megahertz allows a measure of the sense wire signal every 9.4 nanoseconds. The intrinsic rms fluctuations in 


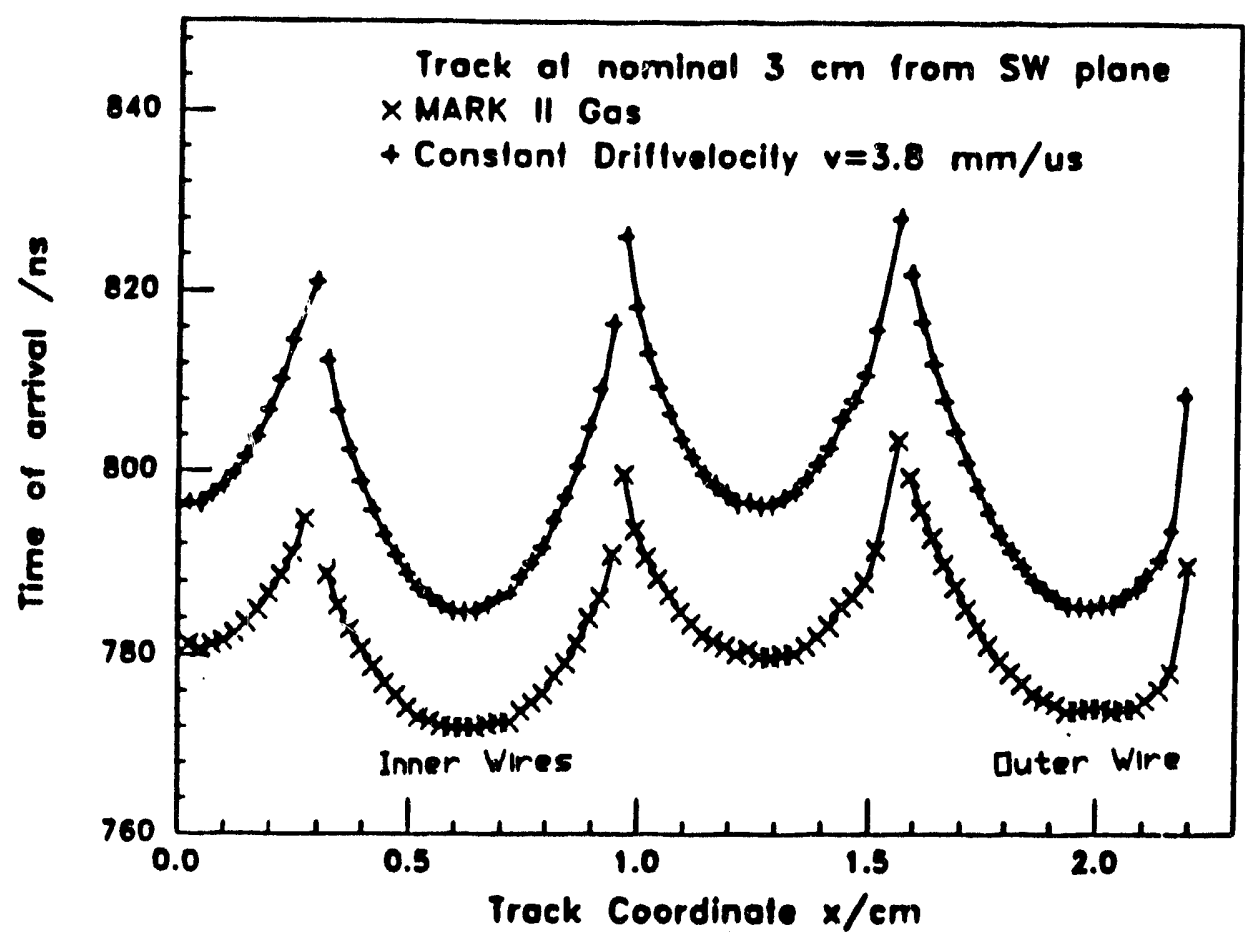

Figure 5.20: Calculated drift time vs ionization position along the sense wire gap for $3 \mathrm{~cm}$ of drift.

the measured arrival time due to the discrete nature of the sampling device was determined by parameterizing a typical sense wire pulse and displacing its position in time by small increments with respect to the 9.4 nanosecond sampling rate. The dispaced pulse is then sent through the hitfinding algorithm.

Figure 5.22 shows the difference between the hitfinder measured and actual time displacement versus the actual time displacement of the parameterized signal. The rms fluctuation in measured arrival time due to electronic effects is found to be $.5 n$ s which translates into a positional fluctuation of $\approx$ $20 \mu m$.

The total contribution $\sigma_{t}$ to the constant term from both dispersion $\sigma_{d}$ and electronic $\sigma_{e}$ effects is then given by the standard expression

$$
\sigma_{t}=c_{1}=\sqrt{\sigma_{d}^{2}+\sigma_{e}^{2}}
$$

and yields $\sigma_{t i}=54 \mu \mathrm{m}$ and $\sigma_{t o}=66 \mu \mathrm{m}$ respectively for inner and outer 

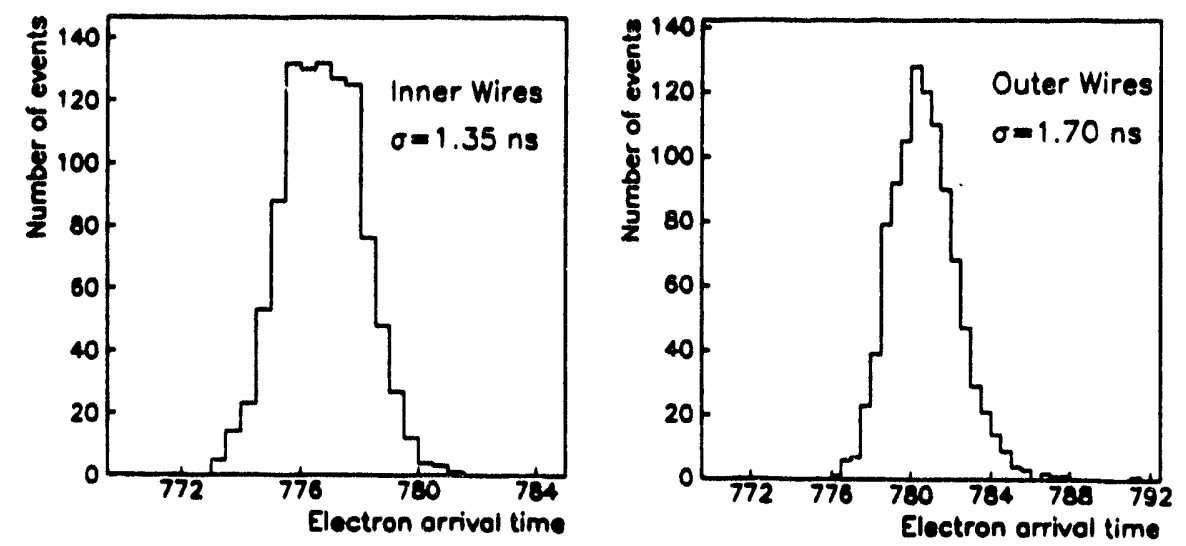

Figure 5.21: Mean arrival time distribution for inner and outer sense wires at $3 \mathrm{~cm}$ of drift showing effects of dispersion.

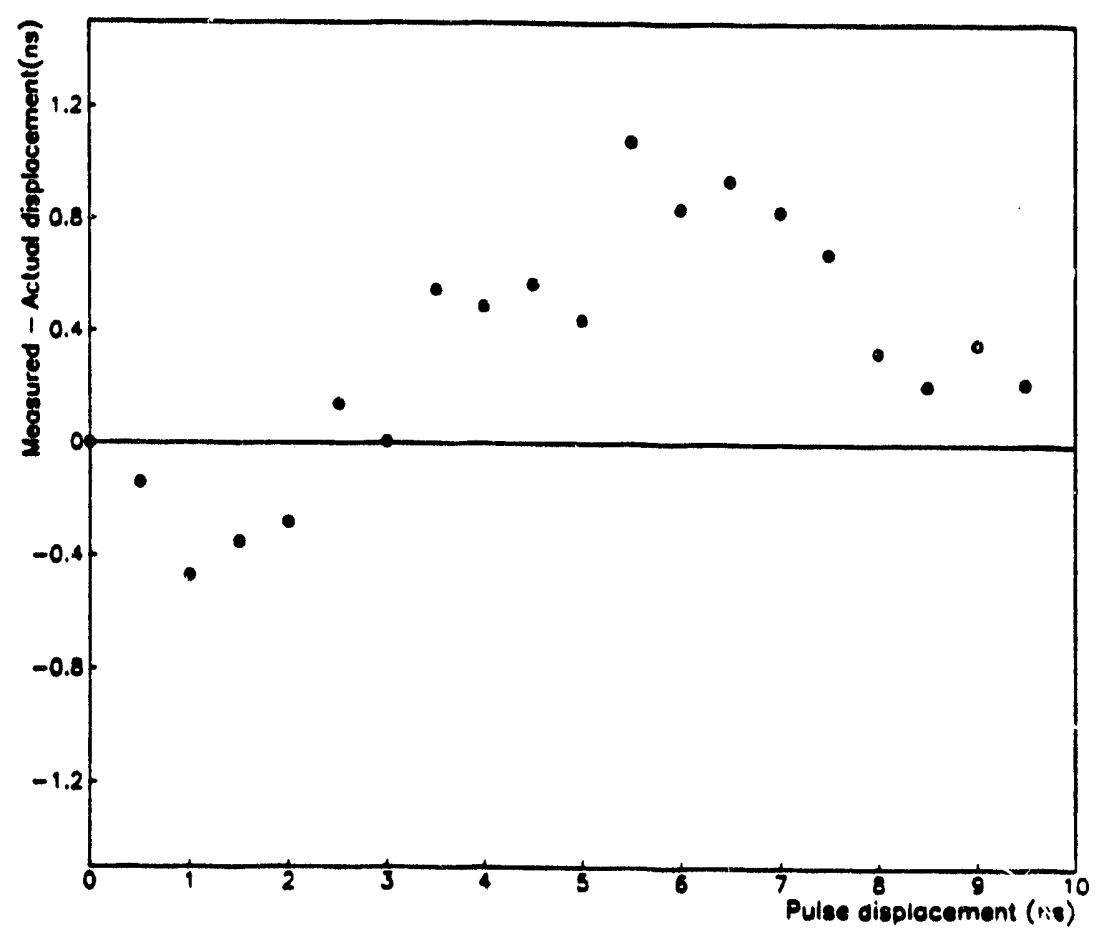

Figure 6.22: Measured pulse arrival time versus displacement. 
sense wires.

\subsubsection{Diffusion Term}

As the drift distance increases, the dominant contribution limiting the chamber resolution is the physical diffusion of the electron swarm along the drift direction. The spread in the electron swarm after drifting for a time $t$ in a constant electric field is given be equation 3.22. If one assumes that the mean arrival time of the electron swarm is the quantity which is actually measured, then the limit imposed by diffusion is given by

$$
\sigma_{x}=\sqrt{\frac{2 D(E) X}{N V_{d}}}
$$

where $N$ is the average number of electrons which drift toward a single sense wire, $V_{d}$ is the drift velocity, $X$ is the drift distance, and $D(E)$ is the field dependent drift coefficient. The expected drift velocity and diffusion coefficient were determined from an analytical solution of equations 3.17 and 3.23 respectivel ${ }^{20}$ for the chamber gas mixture at standard temperature and pressure and with $E=640 \mathrm{~V} / \mathrm{cm}$. It was found that $V_{d}=37 \mu \mathrm{m} / \mathrm{ns}$ in good agreement with the measured drift velocity (figure 5.6) and $D=537 \mu m^{2} / n s$. Using these values along with $N \approx 54$ for the inner wires and $N \approx 64$ for the outer wires (90. total ionization electrons per $\mathrm{cm}$ in argon times a $.6 \mathrm{~cm}$ and $.71 \mathrm{~cm}$ effective charge collection length for the inner and outer sense wires respectively) and comparing equation 5.17 to equation 5.16 gives

$$
\begin{aligned}
c_{2}=\sqrt{\frac{2 D}{N} \frac{D}{V_{d}}} & =74 \mu \mathrm{m} / \sqrt{c m} & & \text { Inner Wires } \\
& =67 \mu \mathrm{m} / \sqrt{c m} & & \text { Outer Wires }
\end{aligned}
$$

\subsubsection{Effect of Angled Segments and Nonlinearities}

Ionizing tracks making a non zero angle with respect to the sense wire plane (figure $5.23 \mathrm{a}$ ) will liberate electrons which are dispersed along the direc- 


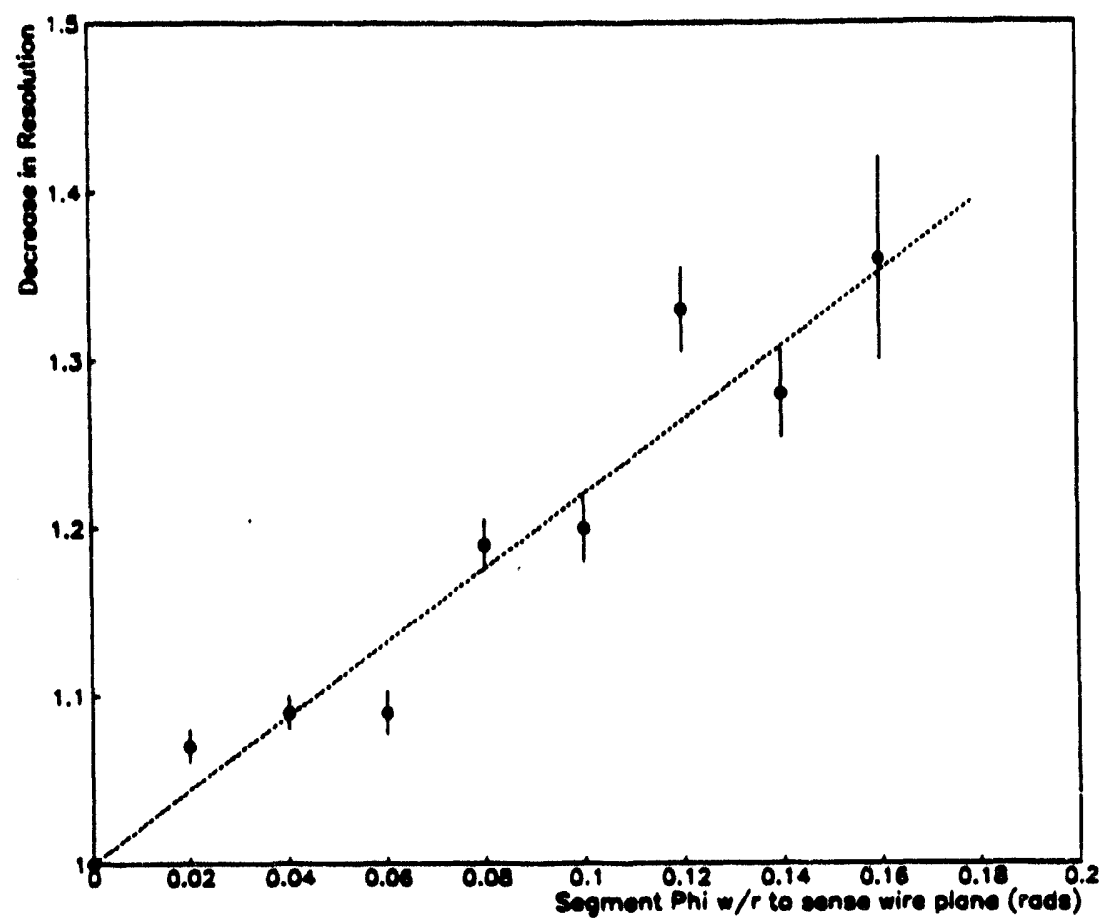

Figure 5.23: Decrease in resolution for track segments making an angle $|\Phi|$ with respect to the sense wire plane.

tion of drift, thereby, widening the arrival time distribution of the individual electron clusters and increasing the effects of dispersion. This effect is man:fested as a deterioration of the sense wire resolution as the angle $\Phi$ is increased. Also, since segments with nonzero $\Phi$ span some length along the drift direction, the existence of drift distance dependent non linearities in the drift velocity will contribute additionally to the sense wire resolution.

Figure 5.23 shows the increase in the width of the inner sense wire residual distribution (decrease in resolution) as a function of $|\Phi|$ for a sample of cosmir ray events restricted in a band between $2.5 \mathrm{~cm}$ and $3.5 \mathrm{~cm}$ from the sense wires. The data is normalized to the measured resolution at $|\Phi|=0$ and the slope of the fitted line is found to be $M=2.2 \mathrm{rads}^{-1}$. From the results of section 5.5.1 figure 5.12, it is seen that this central region of the drift cell is fairly linear for he inner sense wires and any $\Phi$ dependence on the resolution can be assumed 
to result only from dispersion.

The contribution to the chamber resolution from the $\Phi$ dependent dispersion effects was included in the Monte Carlo simulation by introducing a fluctuation term of the form

$$
\sigma^{\prime}(\Phi)=\sigma(X=3 \mathrm{~cm}) \sqrt{(1+2.2|\Phi|)^{2}-1}
$$

where the scale term $\sigma(X=3 \mathrm{~cm})$ is the predicted Monte Carlo resolution (equation 5.16) for $\Phi=0$ at a drift distance of $3 \mathrm{~cm}$. Also included were the nonlinear drift velocity effects based on the parameterization of the residual curves given in table 5.1 .

The Monte carlo result with the addition of $\Phi$ dependent effects and nonlinear effects is shown in figure 5.18 as the cosmic ray Monte Carlo curves. The expected inner sense wire resolution is comparable to the cosmic ray data to generally better than $15 \%$ for the drift region $X>1 \mathrm{~cm}$. The expected outer sense wire resolution, while not as quantitatively accurate as the inner wire curve, retiect's the significant effect of nonlinearities in degrading the outer wire resolution.

\subsection{Two Track Resolution in $R \Phi$}

The efficiency with which ihe central tracking chamber can distinguish between two closely spaced $R \Phi$ ionizing tracks depends largely on the pulse shaping characteristics, intrinsic electronic noise, and optimization of the software hitfinder thresholds. Based on a typical channel's baseline fluctuation of 1.5 FADC counts for the cosmic ray data, values of 3 and 7 FADC counts respectively were used for the $\Delta_{1}$ and $\Delta_{2}$ hitfinder thresholds (section 2.3). Pulses with a peak amplitude of less than 10 counts were excluded from the analysis as were pulses which saturated the dynamic range of the FADCs. 


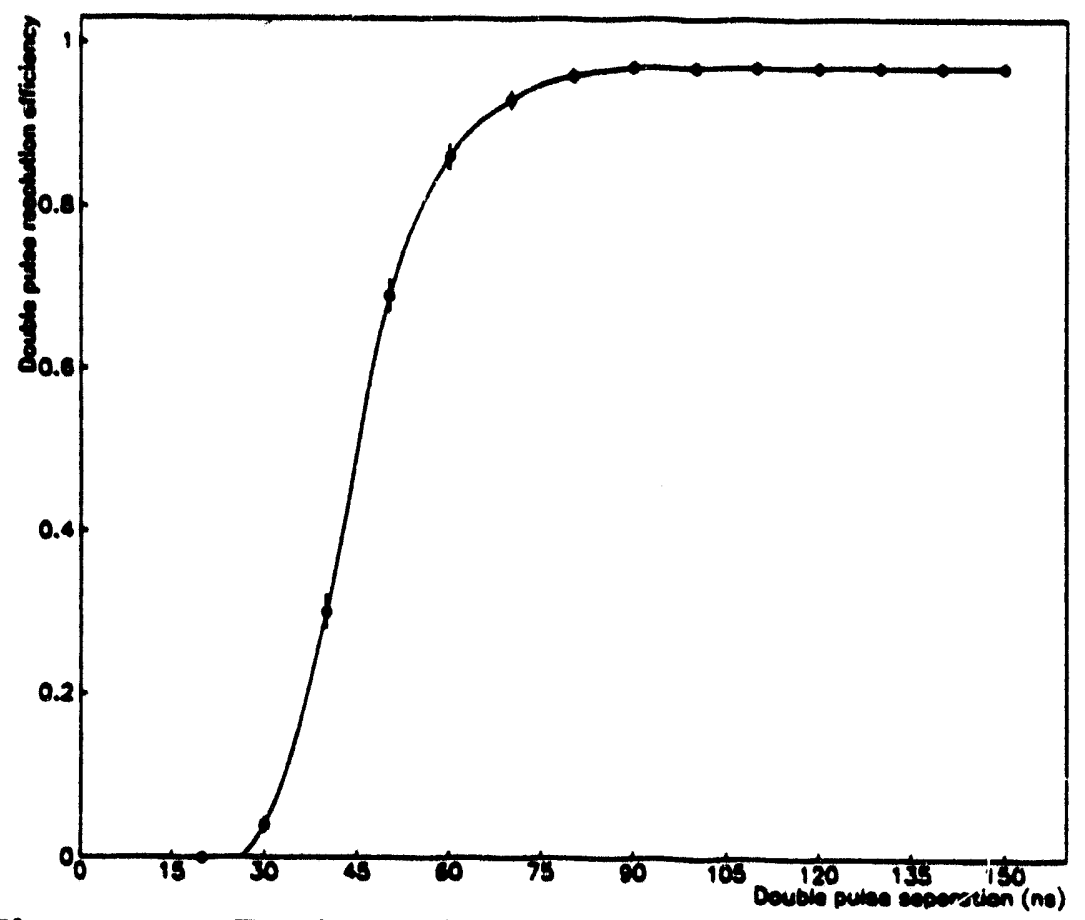

Figure 5.24: Two hit resolution efficiency versus pulse seperation.

The two track resolution efficiency was determined by taking a random sample of pulses involved in full $R \Phi$ tracks. Two such pulses were superimposed on top of each other while being displaced in time by increments of $10 \mathrm{~ns}$. The resulting pulse shapes were then sent through the software hitfinder. Figure 5.24 gives the efficiency for resolving the two pulses as a function of the pulse seperation. The $50 \%$ efficiency level is achieved at a pulse seperation of $45 \mathrm{~ns}$ or $1.7 \mathrm{~mm}$, and $90 \%$ efficiency is at $65 \mathrm{~ns}$ or $2.5 \mathrm{~mm}$.

\section{$5.9 d E / d X$}

The ability of the central tracking chamber to sample the ionization of a track up to 28 times ( 7 sense wires $\times 4$ layers) enables it to accept true single particle tracks and reject double particle tracks generated by photon coversions into overlapping $e^{+} e^{-}$pairs within the detector material. Since no 
double track events were available, they were simulated by superimposing two consecutive single track events with the acceptance criteria for an event the same as described in section 5.7. The ratio of 4 segment to 3 segment tracks in the data sample was 4 to 1.

The ionization of single track and simulated double track events was taken as the mean charge (pulse area) averaged over all sense wires participating in the track after normalizing each wire's charge distribution and scaling by $\sin \theta$ (figure 2.2) to compensate for the effective charge collection length seen by the sense wires (figure $5.28 \mathrm{a}$ ).

The normalization factor for each wire was taken to be the scaled harmonic mean of the charge distribution $\langle 1 / Q\rangle$ defined as

$$
\left\langle\frac{1}{Q}\right\rangle=\sum_{i=1}^{N} \frac{1}{q_{i} \sin \theta_{i}}
$$

where $q_{i}$ is the collected charge and $\theta_{i}$ is the polar angle of the track for the $i^{\text {th }}$ event. Normalizing with respect to the harmonic mean biases against the long tails of the Landau distributions while favoring the bulk of the events. A typical sense wire charge distribution after normalization is shown in (figure $5.28 b)$.

Figure 5.25 shows the resulting distributions for the $70 \%$ truncated mean (discussed below) single track (1MIP) and double track (2MIP) events taken over all 32 modules of the central tracking chamber. The fact that the 1MIP and 2MIP curves are centered about 1 and 2 respectively is a coincidental effect resulting from taking the mean of the lowest $70 \%$ of charges from a distribution which is normalized to have a unean greater than one. For a given cut, the 1MIP acceptance efficiency is the fraction of the 1MIP area to the left of the cut and the 2MIP rejection is the ratio of the 2MIP area to the right of the cut divided by the 2MIP area to the left of the cut.

The two particle rejection power at both $90 \%$ and $95 \%$ single particle efficiency as a function of the charge truncation factor is shown in figure 5.26. 
For example, the points at $X=1.0$ are obtained by taking an average over all sense wires participating in the track whereas the points at $X=.7$ represent an average using only the smallest $70 \%$ of the sense wire charges. There is a clear improvement in the two particle rejection when a $70 \%$ truncated mean is used. Again referring to figure 5.25, a $95 \%$ efficiency for single particle tracks (1MIP) and a rejection power of $95 \pm 13$ for double particle tracks (2MIP) is obtained with a cut at 1.3 times the $70 \%$ truncated mean of the 1 MIP curve. There is no improvement in rejection power when a correction for the drift distance dependent sense wire charge loss is applied (figure 5.27). 


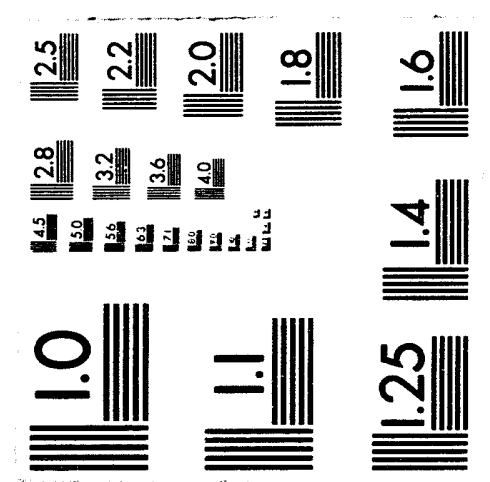



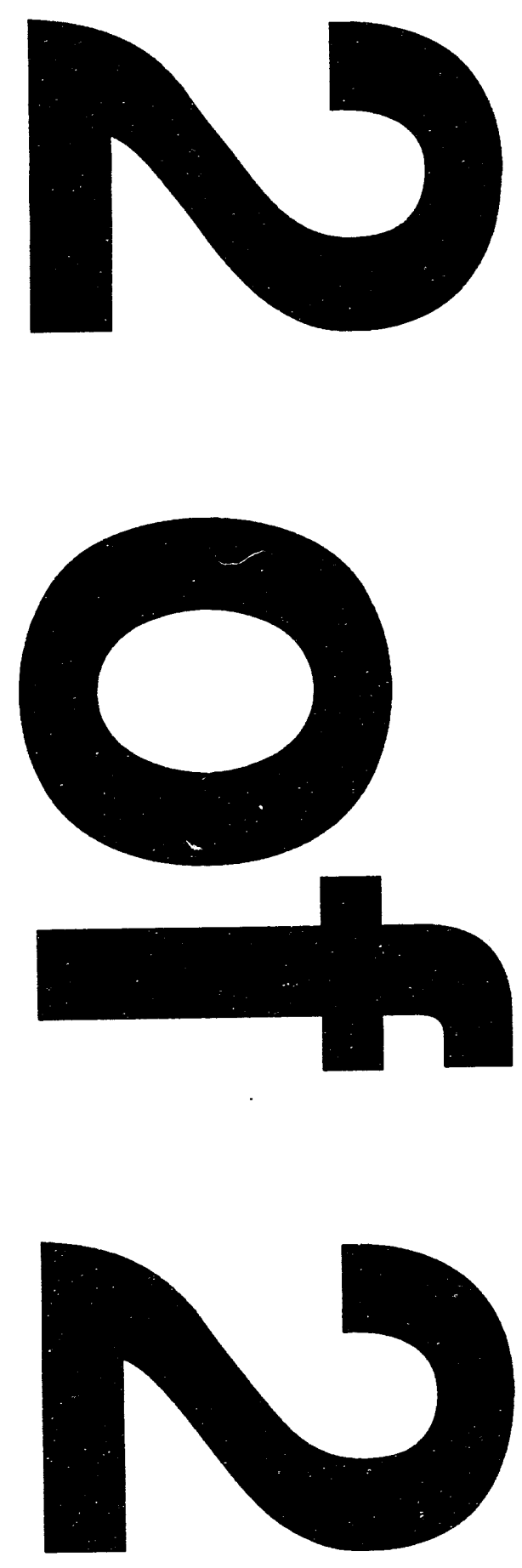
For example, the points at $X=1.0$ are obtained by taking an averags over all sense wires participating in the track whereas the points at $X=.7$ represent an average using only the smallest $70 \%$ of the sense wire charges. There is a clear improvement in the two particle rejection when a $70 \%$ truncated mean is used. Again referring to figure 5.25, a $95 \%$ efficiency for single particle tracks (1MIP) and a rejection power of $95 \pm 13$ for double particle tracks (2MIP) is obtained with a cut at 1.3 times the $70 \%$ truncated mean of the $1 \mathrm{MIP}$ curve. There is no improvement in rejection power when a correction for the drift distance dependent sense wire charge loss is applied (figure 5.27). 


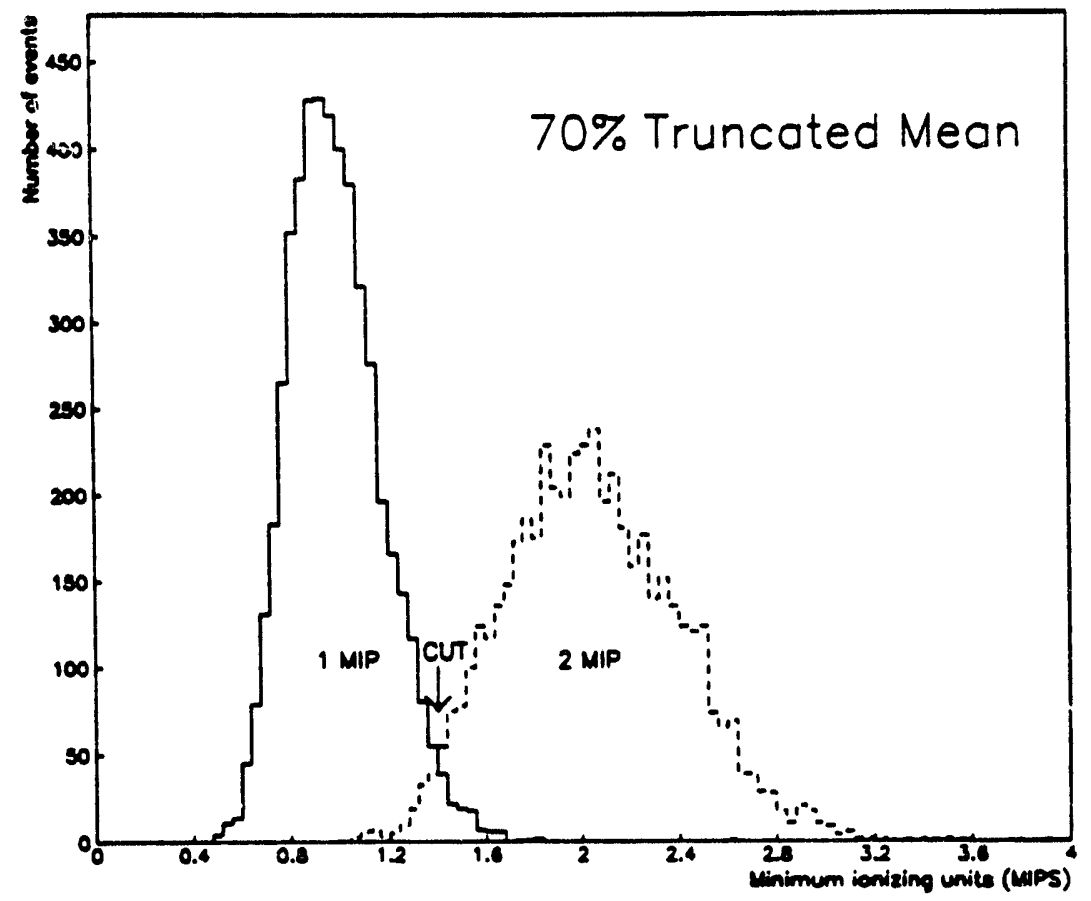

Figure 5.25: Single and double track mean sense wire charge distributions. Each entry in the histogram is an average of the lowest $70 \%$ of the collected sense wire charges.

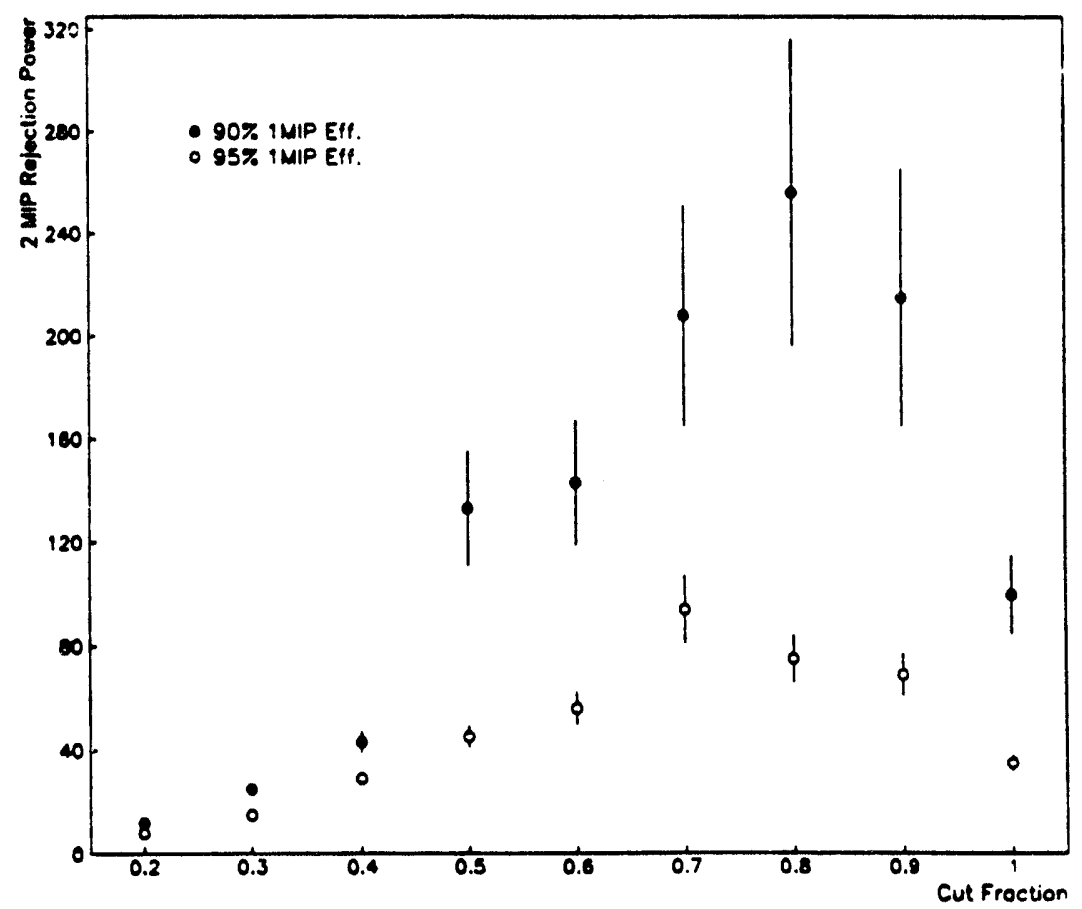

Figure 5.26: Two particle rejection power vs truncated mean. 

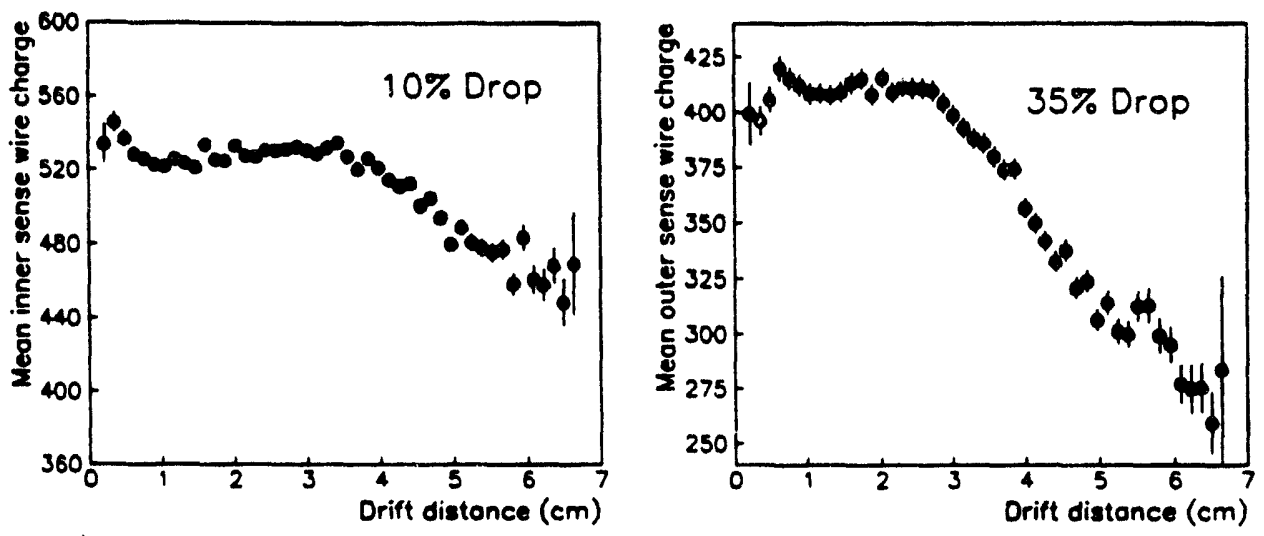

Figure 5.27: Mean sense wire charge vs drift distance for inner and outer wires.
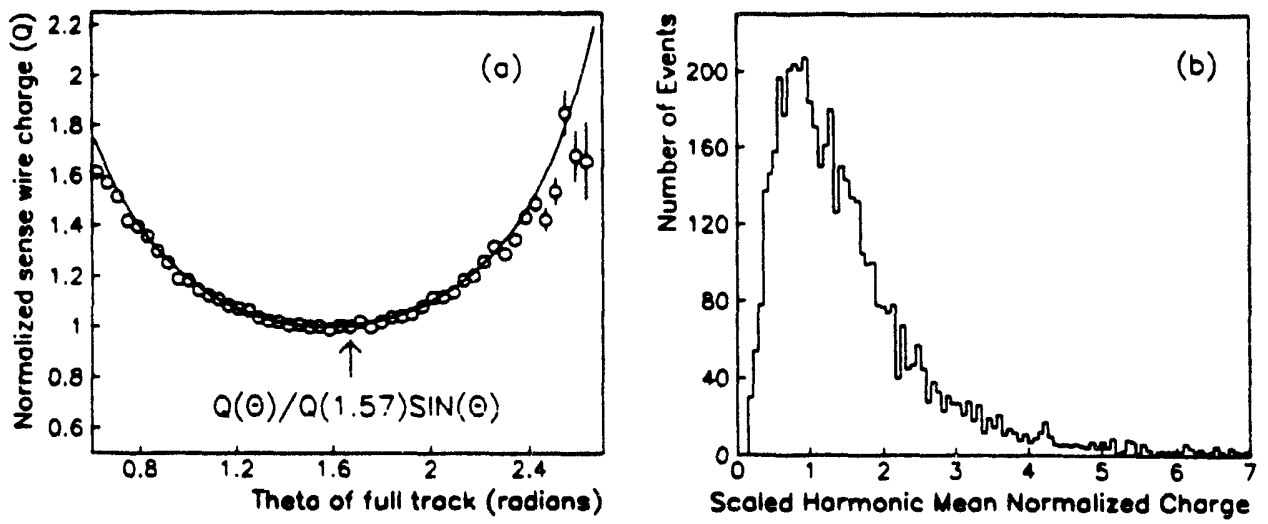

Figure 5.28: (a) Normalized mean charge on sense wires for single track events vs. theta of full track. The data points are normalized with respect to the mean charge at $\Theta=1.57$ radians. (b) Scaled harmonic mean normalized charge distribution for a typical sense wire. 


\subsection{Drift Velocity Studies}

The drift velocities of both inner and outer sense wires were determined for the complete central tracking chamber by selecting a sample of cosmic ray events containing a 4 segment $R \Phi$ track fully contained within a half module (figure 5.7). Two sets of tracks were then formed, one made only from the inner sense wire hits and one made only from the outer sense wires hits. The drift velocity and velocity step (section 5.5.2) for each track type (inner and outer wire) and for each half module was found on an event by event basis according to equations 5.13 and 5.14 . In this way, the mean velocity and mean velocity step were determined from the event sample.

\subsubsection{Mean Velocity and Velocity Step}

Figures 5.29 and 5.30 show the mean velocity of the 4 layers of a half module and the mean velocity step across the layers of a half module for the complete central tracking chamber. The inner and outer sense wire results are plotted seperately and normalized with respect to the average mean velocity taken over all 64 half modules. Two structures are evident in the mean velocity results; a random fluctuation due to changes in atmospheric pressure and temperature during data collection (to be discussed), and a sytematic difference in mean velocity between even and odd numbered half modules. The velocity step results suggest that a small difference in drift velocity between layers exists. An upper limit on the drift velocity difference between two layers is estimated to be $1.5 \%$.

The systematic difference between even and odd half module mean velocities can be understood in terms of the measured nonlinearity functions of section 5.5.1. A Monte Carlo simulation in which the nonlinearities are added to a constant drift velocity predicts a measured mean velocity difference between odd and even modules of $.29 \%$ and $.18 \%$ respectively for inner and outer 

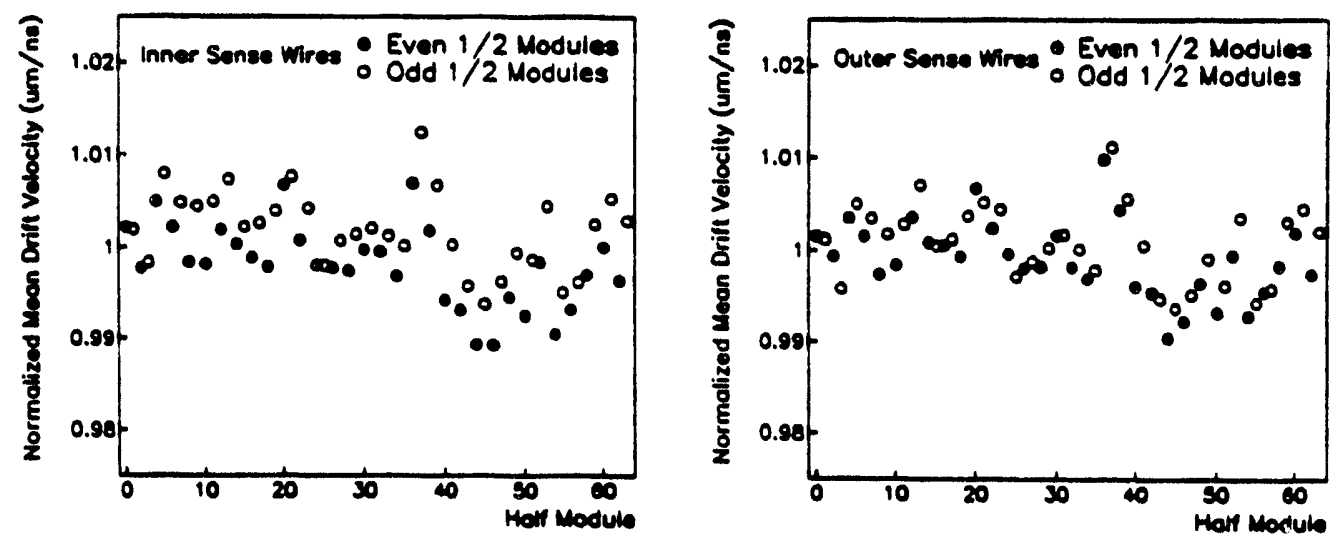

Figure 5.29: Mean velocity of inner and outer sense wires for all 64 half modules of the complete central tracking chamber.
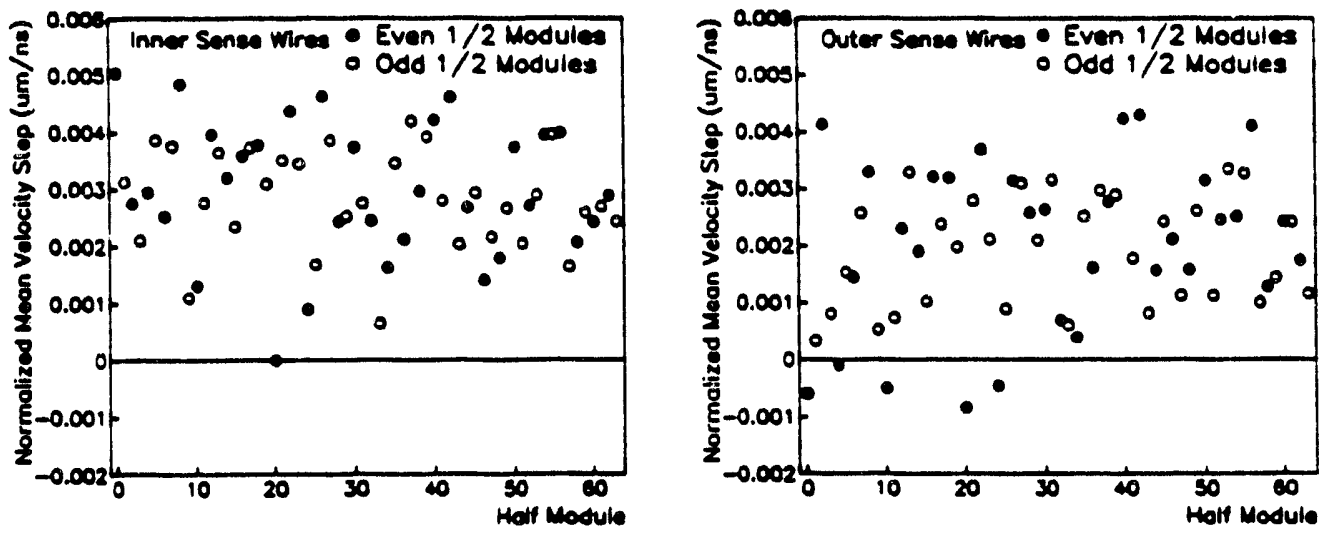

Figure 5.30: Mean velocity step of inner and outer sense wires for all 64 half modules of the complete central tracking chamber. 
sense wires compared with measured differences of $.40 \%$ and $.18 \%$.

The difference between inner and outer wire nonlinearity functions will also generate a difference in the measured mean velocity between inner and outer wires. The Monte Carlo result gives a $.45 \%$ difference with the inner wires acquiring the larger velocity. The cosmic ray data show a difference of $1.4 \%$ and the Fermilab test beam data (figure 5.6) a difference of $.80 \%$. This suggests that the observed drift velocity difference cannot be attributed solely to the shape of the nonlinearity curves. We conclude that for the operating voltages as given in section 5.5 the outer wire drift velocity is less than that of the inner wires by $\approx 1 \%$.

\subsubsection{Pressure and Temperature Dependence}

Changes in pressure and temperature will effect the density of the drift gas and therefore modify the electron mean free path between collisions with the gas molecules (equations 3.18 and 3.19). The electron energy distribution (equation 3.14) and drift velocity (equation 3.17) depend on the electron mean free path and will consequently also be modified. The dependence of the drift velocity on the pressure and temperature of Mark 2 gas was found through an analytical solution of these equations for a drift field $E$ of $640 \mathrm{~V} / \mathrm{cm}$. Figure 5.31 shows the percent change in drift velocity per percent change in pressure and temperature. For standard pressure and temperature, we find

$$
\begin{array}{ll}
\frac{P}{V} \frac{d V}{d P}=+.232 & P \text { in Torr } \\
\frac{T}{V} \frac{d V}{d T}=-.255 & T \text { in Kelvin }
\end{array}
$$

Figure 5.32 shows the measured normalized mean drift velocity of a single half module and the atmospheric pressure as a function of time (arbitrary 

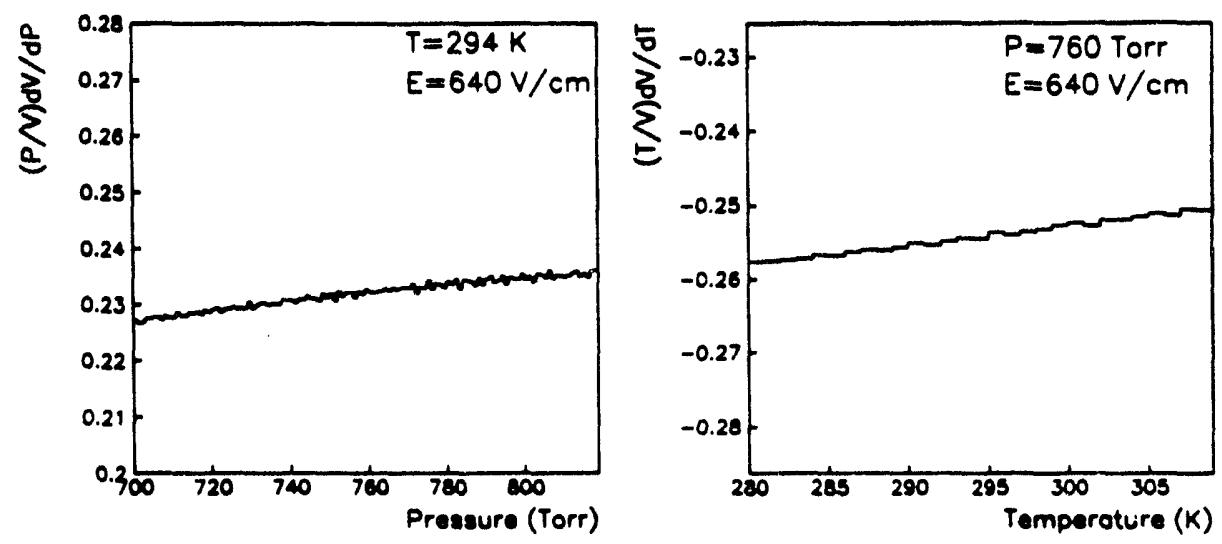

Figure 5.31: $\frac{P}{V} \frac{d V}{d P}$ vs. $P$ and $\frac{T}{V} \frac{d V}{d T}$ vs. $T$ for Mark 2 gas at $E=640 \mathrm{~V} / \mathrm{cm}$ (theory).
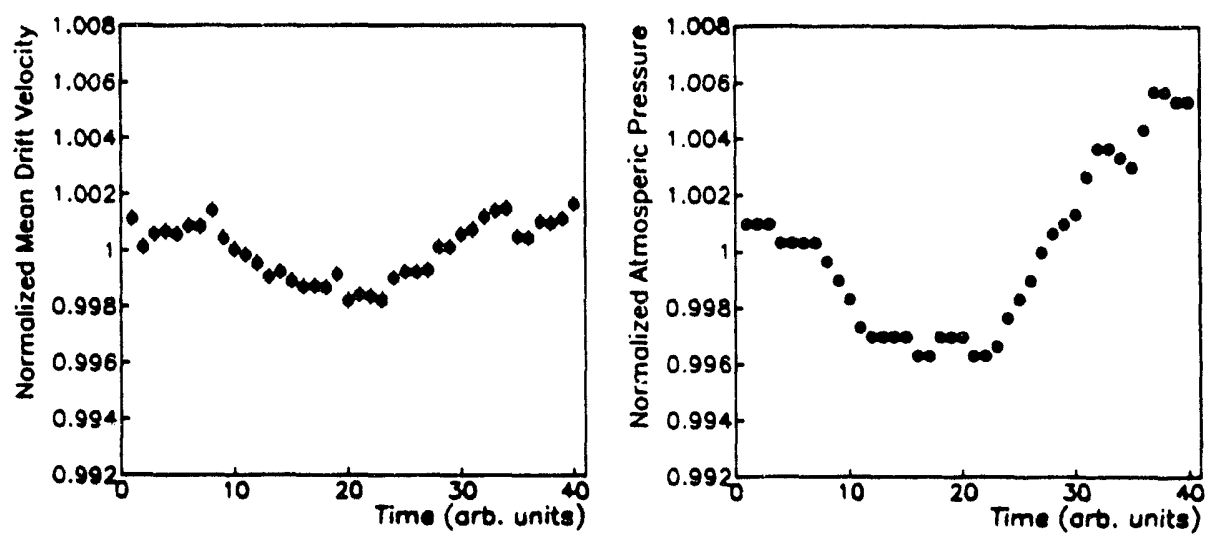

Figure 5.32: Measured normalized mean drift velocity of a single half module and normalized atmospheric pressure versus time (arb. units). 
units) taken over a 72 hour continuous data taking period. The drift velocity is seen to track with the pressure curve, however, a direct confrontation with theory was not possible due to the inavailability of reliable temperature data.

\subsubsection{Gas Mix and $E$ Field Dependence}

The theoretical drift velocity dependence on the components of the gas mix was found through an analytical solution of equation 3.17. The percent change in drift velocity $\% \Delta V$ as the methane $\left(\mathrm{CH}_{4}\right)$ and carbon dioxide $\left(\mathrm{CO}_{2}\right)$ component (Nominal $93 \%$ Argon, $4 \%$ Methane, $3 \%$ Carbon Dioxide) is increased by $1 \%$ from its nominal value (i.e $3 \% \mathrm{CO}_{2}$ to $3.03 \% \mathrm{CO}_{2}$ ) is predicted to be (at standard pressure and temperature)

$$
\begin{aligned}
& \frac{\% \Delta V}{\% \Delta C H_{4}}=.213 \\
& \frac{\% \Delta V}{\% \Delta C O_{2}}=.316
\end{aligned}
$$

and is consistent with measured results using a prototype drift cell. ${ }^{23}$

The expected drift velocity in MARK 2 gas as a function of electric field strength is shown in figure 5.33. The theoretical value of $37 \mu \mathrm{m} / n s$ at $E=$ $640 \mathrm{~V} / \mathrm{cm}$ agrees well with the direct measurements shown in figure 5.6. The percent change in drift velocity per percent change in $E$ is esentially flat in the neighborhood of the nominal field and is found to be

$$
\frac{E}{V} \frac{d V}{d E}=-.232 \quad E=640 \mathrm{~V} / \mathrm{cm}
$$

\subsubsection{Nonlinearities}

The effects of outer sense wire nonlinearities can be understood by examining the mean residual of the outer sense wire hits with respect to a track segment (partial track formed from a single layer) constructed from the inner sense wires only. Figure 5.34 shows the variation of the mean outer wire 


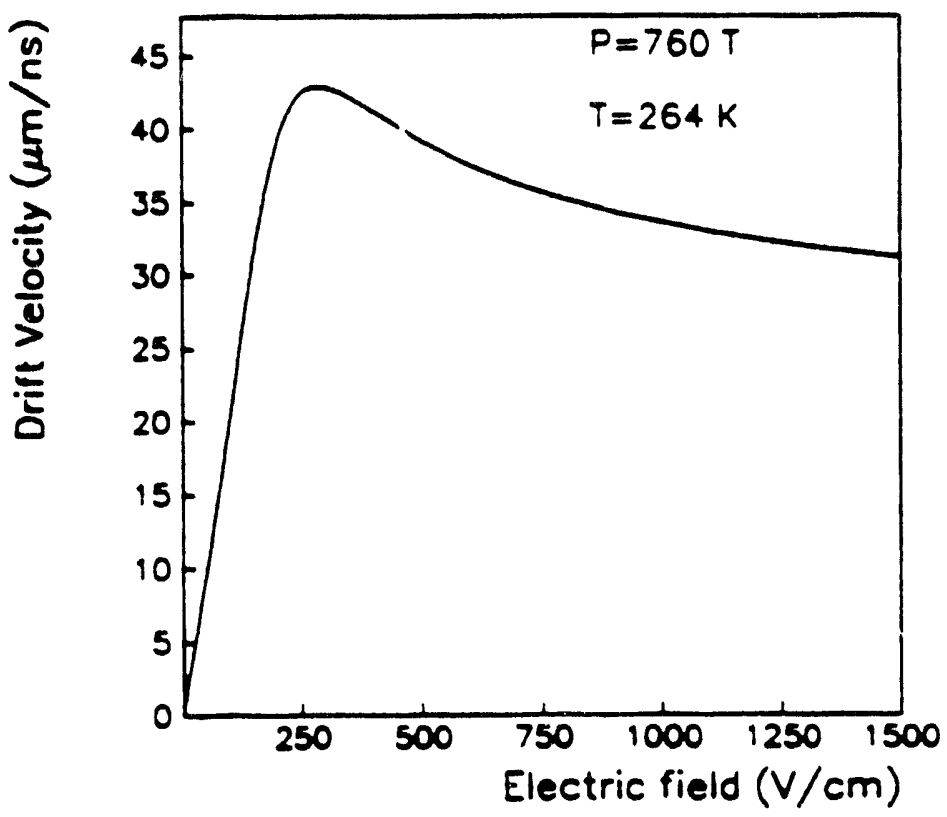

Figure 5.33: Theoretical electron drift velocity vs. electric fielu strength

residual as a function of drift distance for even and odd half modules. The data are averaged over all even and odd half modules of the complete central tracking chamber. Also shown is the expected mean residual variation when the nonlinearity functions of section 5.5.1 are included in the Monte Carlo simulation.

The effect of inner sense wire nonlinearities can be seen by examining the residual between the midpoint of a layer 0 and layer 2 segment with respect to a single track formed from the layer 1 and layer 3 track segments. Figure 5.36 shows the dependence of the mean residuals on the drift distance as measured from the midpoint of the layer 0 and layer 2 track segments after adjustment of the mean drift velocity and drift velocity step. Also shown is the expected shape as predicted from Monte Carlo when the test beam measured nonlinearities (section 5.5.1) are included. The segments and tracks were formed from a fit of the inner sense wires only. 
We see that the Monte Carlo results agree well with the data for both even and odd half modules and for inner and outer sense wires. This suggests that the correction function parameterizations of table 5.1 can be used to improve the tracking resolution especially around the highly nonlinear regions near the anode and cathode where the distortions are largest. Figures $\mathbf{5 . 3 5}$ and 5.37 show the mean residuals after applying the correction functions. The corrected residuals are flat throughout the width of the drift cell with the maximum deviation about zero generally less than 200 microns. 

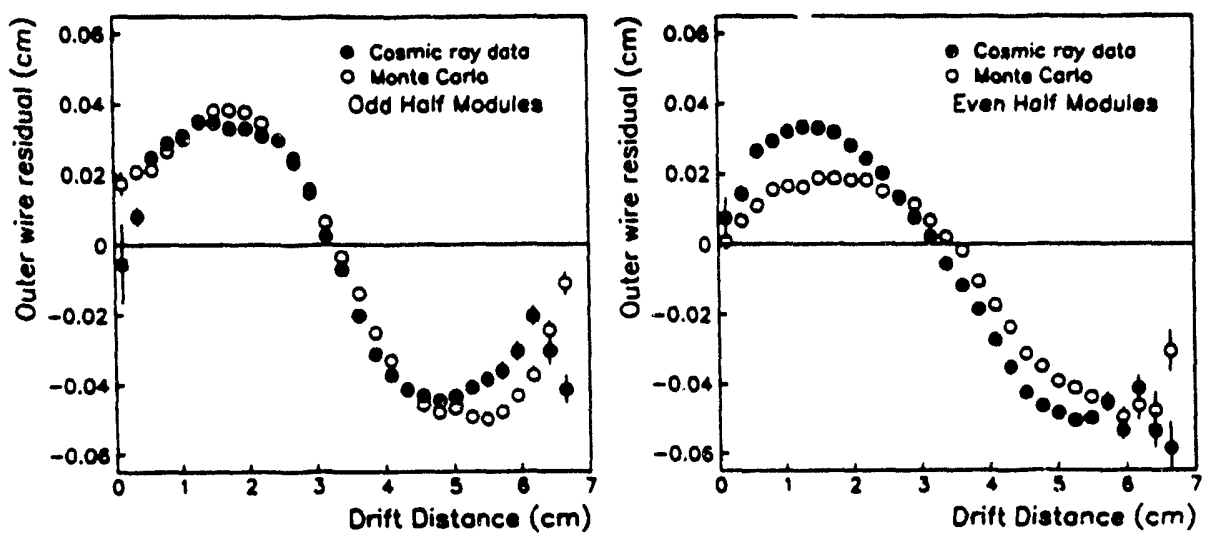

Figure 5.34: Mean residual versus drift distance of outer sense wire hits with respect to a track segment formed from the inner sense wires. Results are before applying drift distance dependent correction functions.
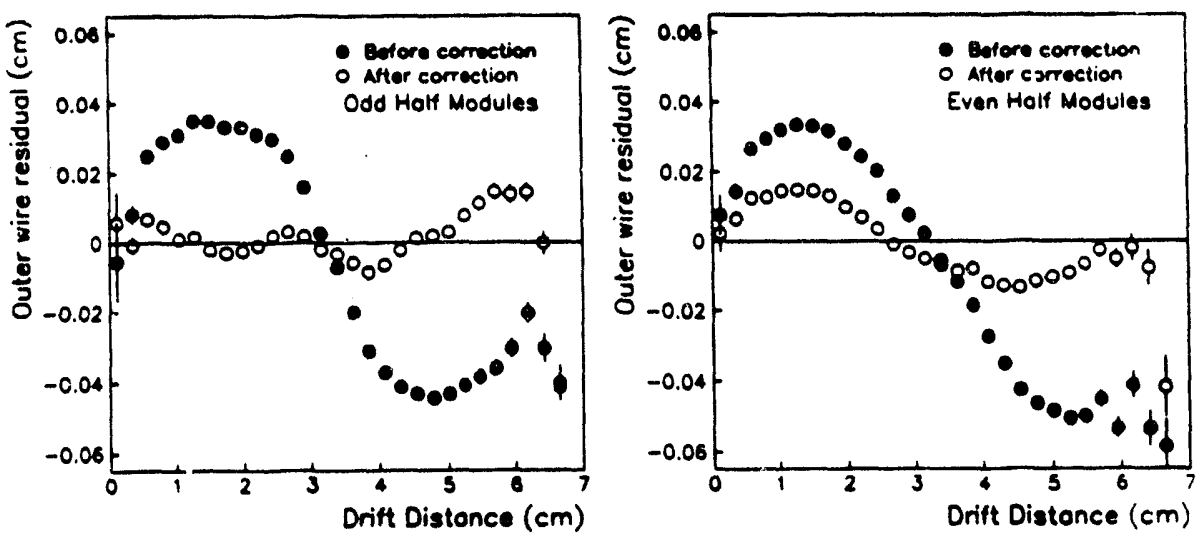

Figure 5.35: Mean residual versus drift distance of outer sense wire hits with respect to a track segment formed from the inner sense wires before and after applying drift distance dependent correction functions. 

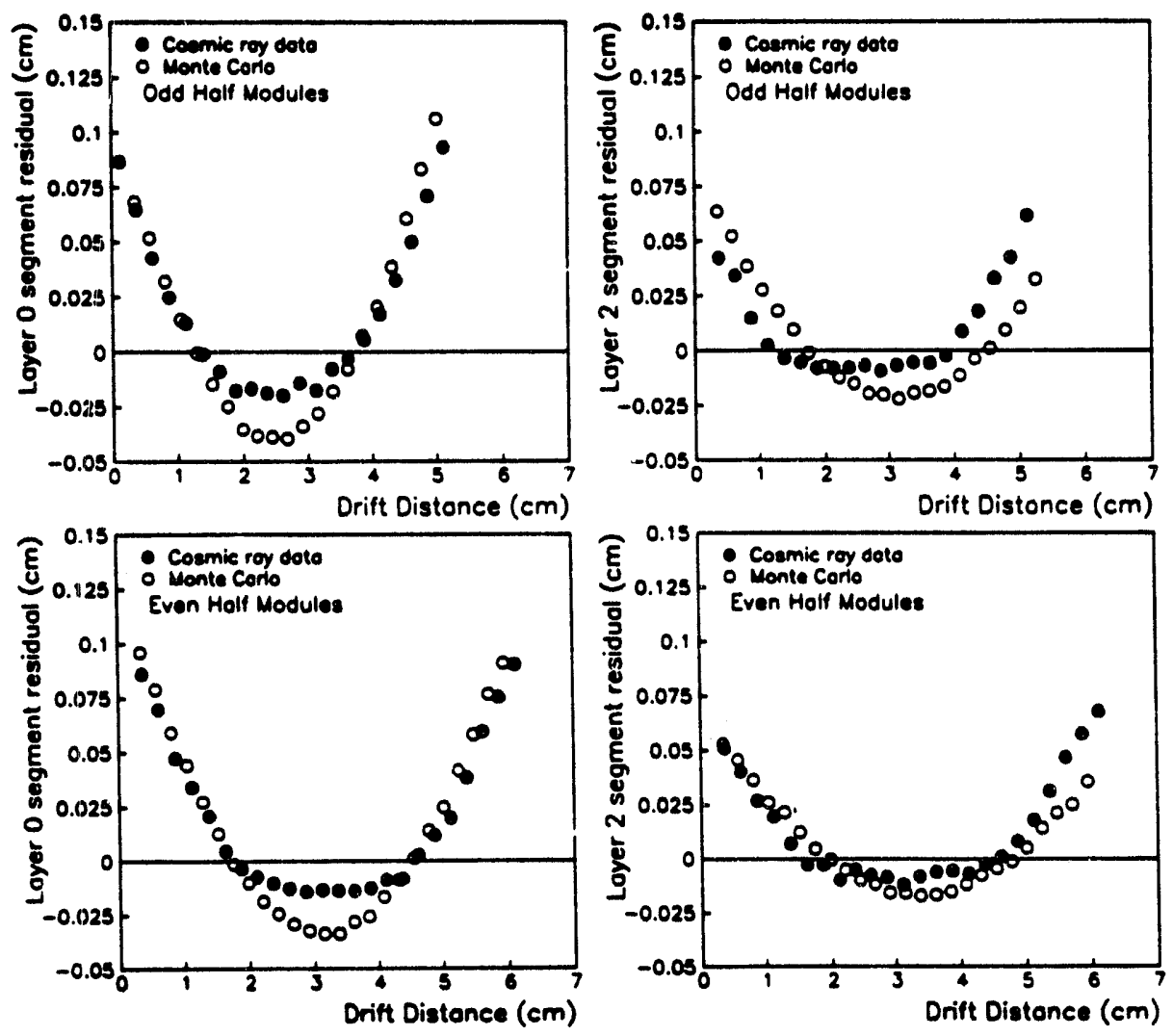

Figure 5.36: Mean residual versus drift distance of layer 0 and layer 2 segment with respect to a 2 segment track formed from layers 1 and 3 . Results are before applying drift distance dependent correction functions. 

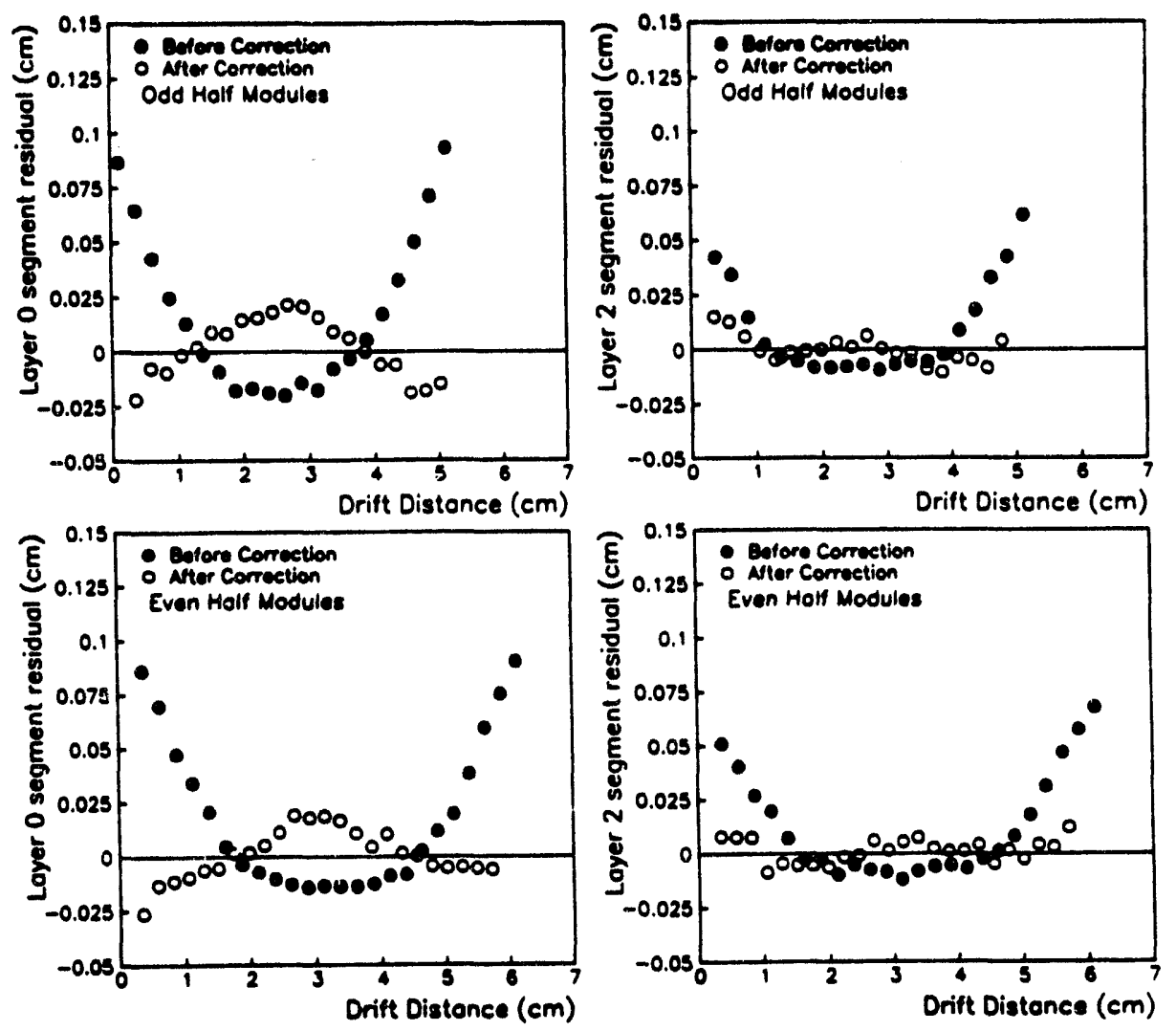

Figure 5.37: Mean residual versus drift distance of layer 0 and layer 2 segment with respect to a 2 segment track formed from layers 1 and 3 before and after applying drift distance dependent correction functions. 


\subsection{Tracking Across 4 Layers}

The ability to accurately reconstruct full tracks spanning all 4 layers of the drift chamber depends crucially on a choice of a correct average drift velocity, an understanding of the effects of nonlinearities in the drift field (sections 5.5.1 and 5.10.4), and a carefull monitoring of drift velocity fluctuations imposed by atmospheric and gas composition changes (section $\mathbf{5 . 1 0 . 2}$ ).

\subsubsection{Alignment Procedure}

The procedure for alignment of the chamber data is a 4 step iterative process. The required data consist of 4 segment tracks which are fully contained within a half module. Most of the tracks generated within the nominal interaction region at D0 are expected to satisfy these alignment criteria. It is assumed that there initially exists a correct set of electronic time offsets for each sense wire. The approach to alignment is as follows:

1. Begining with an approximate initial value for the drift velocity and the best estimate for the overall time delay necessary for a preselection of hits to $r^{\circ}$ rticipate in full tracks, determine the velocity and velocity step for each half module containing a 4 segment track on an event by event basis according to equations 5.13 and 5.14 for the inner and outer sense wire groups. Include the drift distance dependant corrections as shown in figure 5.13 and parameterized in table 5.1. A positive residual at some distance from the anode requires a shifting of the space point away from the anode by the residual amount. Take the mean of each distribution after a specified number of tracks and determine the drift velocity for each layer of the half module. Due to the small width of these distributions (section 5.5.2), a sample of $\approx 10$ tracks within a half module will give the drift velocity to better that $.15 \%$. 
2. Using the velocities obtained from step 1 as the new starting values, obtain an updated set of velocities in the same way while continuing to include the drift distance dependent corrections.

3. Fix the drift velocities based on the updated results of step 2 and determine the mean residual with respect to the track segment (not full track) fit for each sense wire. A hit with a positive residual is defined as being further away from the sense wire than the fitted line. Since each sense wire participates in tracks for 2 adjacent half modules, there will be two such mean residuals per wire.

4. Adjust the time offsets for each wire according to the mean residuals found in step 3 . Repeat step 3.

5. Update the time offsets based on the new set of residuals obtained from step 4.

The above procedure was used to generate the results of section 5.10.4 and the results included in the following section. Steps 1 and 2 are important in the calibration proceedure since they determine the scale (drift velocity), and ultimately, the accuracy to which tracks may be reconstructed. The effect of steps 3 and 4 is to systematically shift the space point by an amount which is typically less than the sense wire resolution. In general, this proceedure will not be required for a proper calibration of the chamber. Note that steps 1 and 2 are necessary only the first time a chamber alignment is performed. Subsequent alignment runs to compensate for small changes in drift velocity due to atmospheric pressure, temperature, or gas composition fluctuations will generally require only a step 1 phase. 


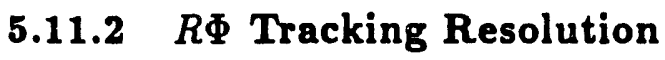

The resolution of a full $R \Phi$ track spanning 4 layers of the central tracking chamber is determined by examining the resolution of the individual sense wires participating in the track fit. Figure 5.38 shows both the average residuals and the resolution (width of residual distribution) for the 28 wires spanning 4 layers of the chamber. The mean residual and resolution with respect to a full track fit using only the inner sense wires are shown seperately for even and odd half modules. The improvement in resolution when using the correction functions is evident especially for the outer sense wires which improve by $\approx$ $33 \%$ for the odd half modules and $\approx 25 \%$ for the even half modules. The improvement for the inner wires is seen from the layer 1 and layer 2 wire groups (wires 8 through 21 ) and is measured to be $\approx 13 \%$ for odd and $\approx 7 \%$ for even half modules. The average inner wire full track resolution after correction is $250 \pm 10 \mu m$ while the average outer wire resolution is worse by $\approx 27 \%$. The small mean residuals (generally less than $40 \mu \mathrm{m}$ ) show that the alignment procedure of section 5.11.1 was succesfull in aligning the seperate segments into full tracks.

\subsection{Monte Carlo Study of Alignment Strategies}

A Monte Carlo analysis of two different alignment strategies was done to determine the power of each method in reconstructing a full $R \Phi$ track. The first method is the one currently employed in the DO software while the second method is the one outlined in section 5.11.1. The current strategy involves using a fixed velocity for all wires and adjusting the individual wire's time offset based on the mean residual with respect to a fit of the full $R \Phi$ track. The Monte Carlo assumed each wire to have a side dependant drift velocity according to the results of section 5.5.1 (figure 5.6). The sense wire resolution 

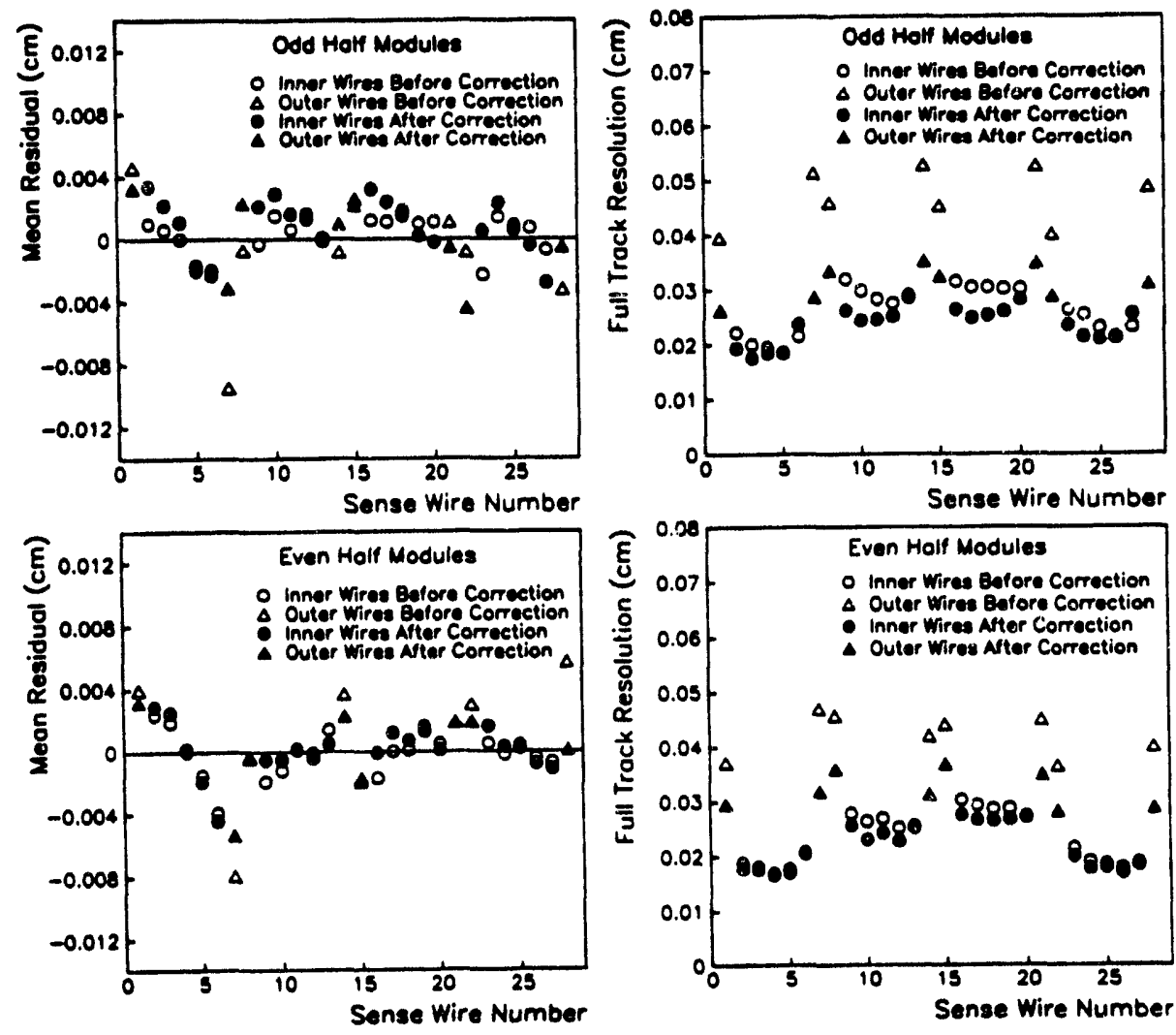

Figure 5.38: Mean residuals and resolution with respect to a 4 layer track fit of the inner sense wires before and after corrections. 
as a function of drift distance was included in the Monte Carlo according to the parameterization given in table 5.7.1.

A sample of tracks originating from the nominal interaction region was generated, reconstructed, and aligned according to both alignment methods. The set of aligned data was compared to the real Monte Carlo track at two points - the transition radiation detector (TRD) - central drift chamber (CDC) interface and at the front face of the electromagnetic calorimeter (EMC). It is assumed that the channel to channel electronic time delays (offsets) are correctly measured and therefore do not contribute to any systematic shifting of the reconstructed track.

Figure 5.39 shows the difference between the recontructed track and real track extrapolation at the TRD-CDC and CDC-EMC interface for two initial values of drift velocity $\left(V_{i}=39 \mu \mathrm{m} / n s\right.$ and $\left.V_{i}=36 \mu \mathrm{m} / n \mathrm{~s}\right)$ and for a drift velocity of $36 \mu \mathrm{m} / \mathrm{ns}$ in conjunction with an error in the choice of overall time offset $(\Delta T 0)$ of $-20 n s$. The results show that the pointing accuracy of the current alignment method depends strongly on the choice of drift velocity and overall TO. On the other hand, the mean of the distributions when using the new alignment method are insensitive to the choice of initial parameters. We see that for a fixed choice of overall To, the new method converges to a fixed drift velocity independantly of the chosen initial velocity. If the overall T0 is incorrectly chosen, then the new method converges to the drift velocity which preserves the mean pointing accuracy. The systematic shift of $\approx 300 \mu \mathrm{m}$ between the real and reconstructed tracks is the result of two effects. First, there exist small drift velocity differences between inner sense wires in the same side of a layer (figure 5.6) which the alignment method does not take into account. Second, the assumption of a $\Delta V$ drift velocity structure between the layers of a half module (section 5.5.2) is not strictly satisfied.

We conclude that the new alignment method is superior to the one cur- 
rently used since it is able to correctly align without the need for an external measurement of the drift velocity or a precise value of the overall time delay. Also, changes in drift velocity may be independantly monitored and compensated for, thereby, eliminating the systematic effects of atmospheric, drift field, and gas composition changes on track reconstruction. For a well measured set of electronic delays, the systematic pointing error at the detector interfaces is estimated to be $\pm 300 \mu m$ with a rms fluctuation of $200 \mu m$. 

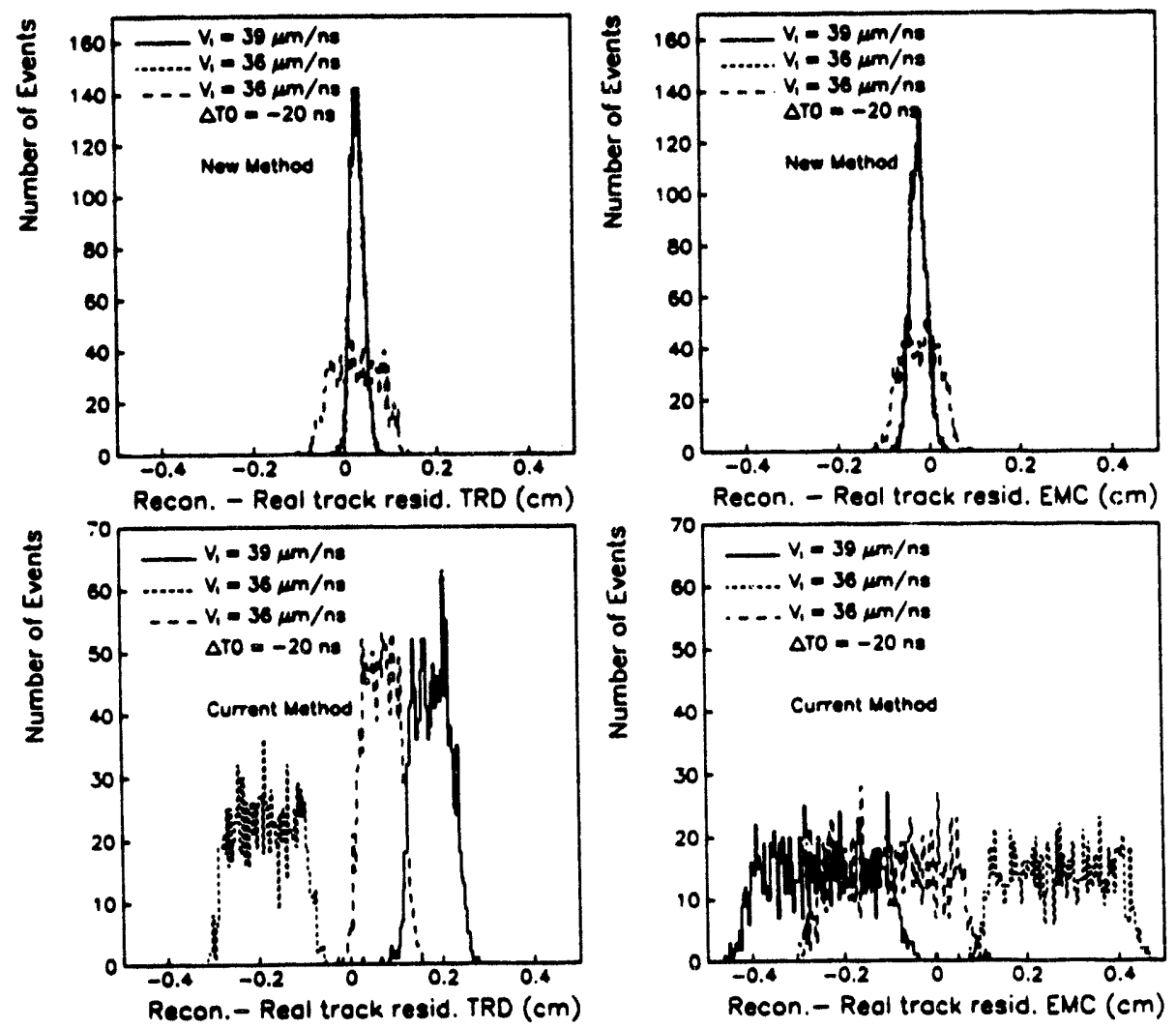

Figure 5.39: Comparison of alignment strategies showing difference between reconstructed and real Monte Carlo track at TRD and EMC boundaries for various values of initial parameters. 


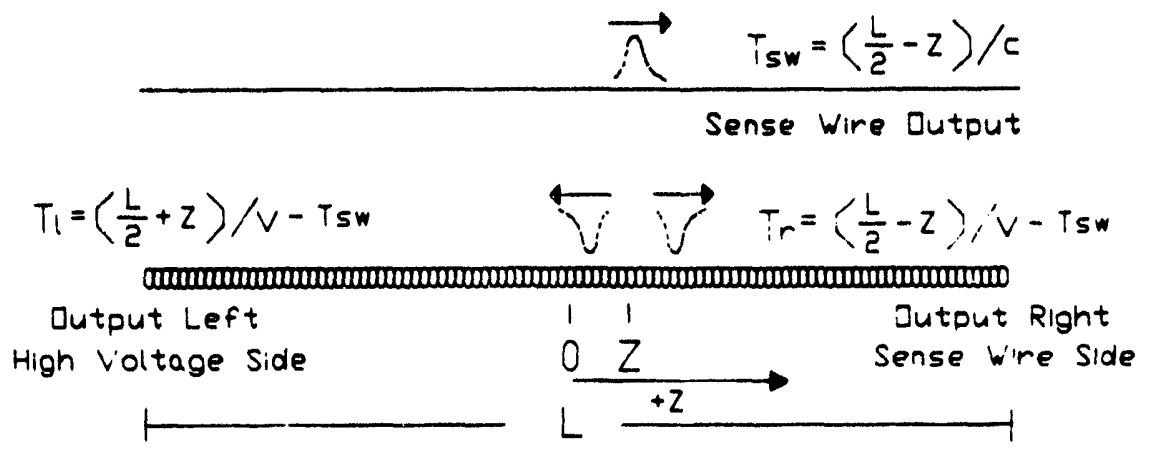

Figure 5.40: Simplified schematic of delay line operation.

\subsection{Measuring Z With Delay Lines}

The delay lines used in the Do central tracking chamber are passive position sensing detectors which rely on an induced pulse generated by the neighboring sense wire. The pulse (with opposite polarity of the sense wire signal) is induced at some position $Z$ on the delay line and begins travelling toward both ends of the line where it is then amplified and shaped before being digitized by the analog to digital converters (FADCs). Taking the sense wire signal arrival time at the FADC as the time origin, the delay line signal left and right side arrival times $T_{l}$ and $T_{r}$ (figure 5.40) are

$$
\begin{aligned}
& T_{l}=(L / 2+Z) / V \\
& T_{r}=(L / 2-Z) / V
\end{aligned}
$$


where $L$ is the full delay line length and $V$ is the signal propagation velocity across the delay line. Solving equations 5.18 and 5.19 for $Z$ gives

$$
\begin{aligned}
& Z_{l}=V\left(T_{l}-\frac{L}{2 V}\right) \\
& Z_{r}=-V\left(T_{r}-\frac{L}{2 V}\right)
\end{aligned}
$$

where $Z_{l}$ and $Z_{r}$ are the $Z$ coordinates as measured from the left and right sides of the delay line. The constant term $L / 2 \mathrm{~V}$ is the signal travel time starting from the line center at $Z=0$ to $Z= \pm L / 2$.

Equations 5.20 and 5.21 are valid in the approximation that the velocity of signal propagation along the sense wire is infinite. In general the existence of a finite propagation velocity $(v=c)$ will result in a $Z$ dependance on the measured sense wire time and, therefore, in the time origin of the delay line signals. Consider a pulse generated at $Z=0$. The measured arrival time $T_{0}$ of the left and right side delay line signals is

$$
T_{0}=\frac{L}{2 V}-\frac{L}{2 c}
$$

For a pulse generated at $Z$ we then have for the left and right side

$$
\begin{aligned}
& T_{l}=T_{0}+\frac{Z}{V}+\frac{Z}{c} \\
& T_{r}=T_{0}-\frac{Z}{V}+\frac{Z}{c}
\end{aligned}
$$

which after solving for $Z$ gives

$$
\begin{aligned}
& Z_{l}=\frac{V}{1+V / c}\left(T_{l}-\frac{L}{2 V}(1-V / c)\right) \\
& Z_{r}=-\frac{V}{1-V / c}\left(T_{r}-\frac{L}{2 V}(1-V / c)\right)
\end{aligned}
$$

Comparing equations 5.22 and 5.23 to 5.20 and 5.21 we see that the effect of a finite signal propagation velocity along the sense wire may be compensated for by introducing a side dependant delay line velocity along with an additional side independant time offset. 
In general, requiring that $Z_{l}$ and $Z_{\tau}$ be within the geometric constraints of the drift cell $(-L / 2<Z<L / 2)$ and that $\left|Z_{l}-Z_{R}\right|$ is a minimum and less than some suitably chosen threshold, are sufficient in determining the correct association between sense wire and delay line pulses. For events in which only a single hit appears on the sense wire and delay line sides this association becomes trivial. For events in which multiple sense wire hits generate multiple delay line signals, the above cuts are still an effective method for sense wire to delay line hit association, however, cases may arise in which the $Z$ difference cut is satisfied by the wrong left and right side pair. In this case, there must always remain at least one pair of delay line pulses which fail this cut. The correct sense wire to delay line hit association is, therefore, given ivy the combination which maximizes the number of left right pairs satisfying the above cuts.

\subsection{Delay Line Calibration}

The delay lines were calibrated by inducing a pulse at 16 positions along each line and measuring the arrival times at both ends (figure 5.41). A linear fit of the $Z$ position versus time as measured from the sense wire side $\left(T_{r}\right)$ gave the mean propagation velocity $(V)$ along the line. A time offset was computed seperately for each side of the delay line to compensate for the difference between the physical center of the line and the geometric center at $Z=0$ after installation into the drift module. Note that from equations 5.18 and 5.19 one can derive an expression

$$
T_{l}+T_{r}=\frac{L}{V}
$$

relating the pulse arrival times at both ends of the line.

The time offset for the sense wire side $\frac{L}{2 V}$. was determined from the ratio of the $Z$ intercept and $V$ (figure 5.42). The high voltage (left) side offset $\frac{L}{2 V} \mid l$ 


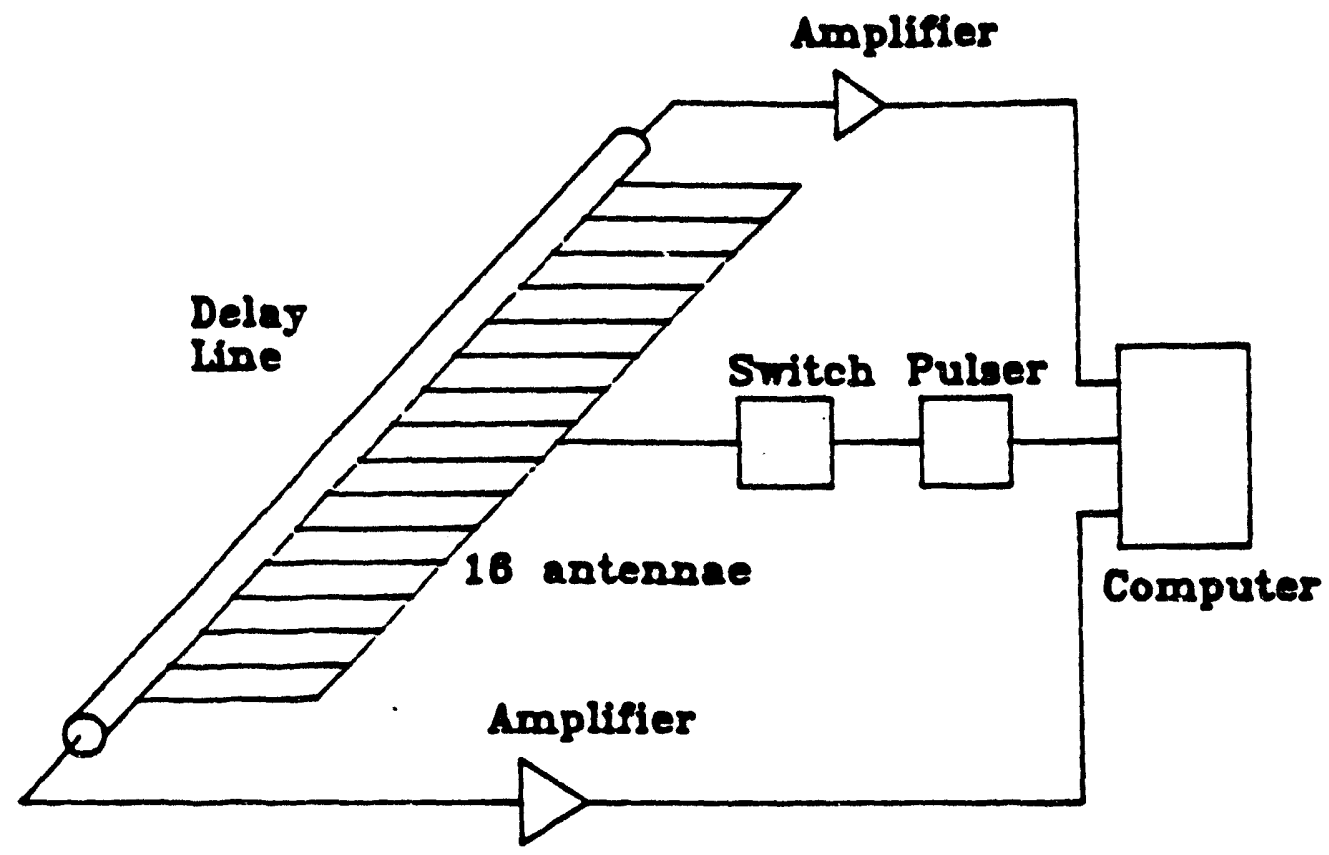

Figure 5.41: Simplified schematic of delay line calibration system.

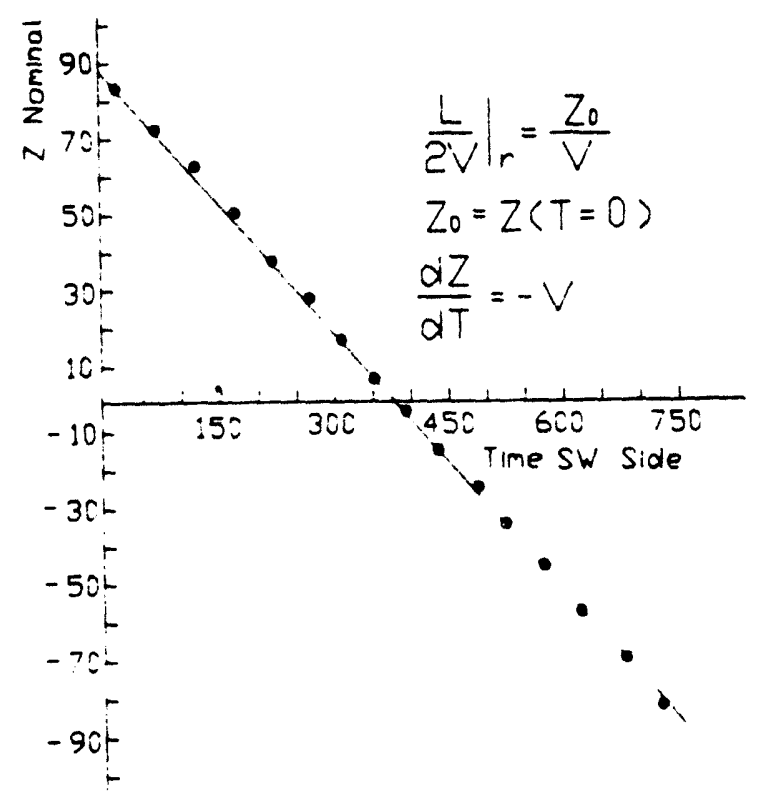

Figure 5.42: Determination of delay line velocity and sense wire side time offset. 

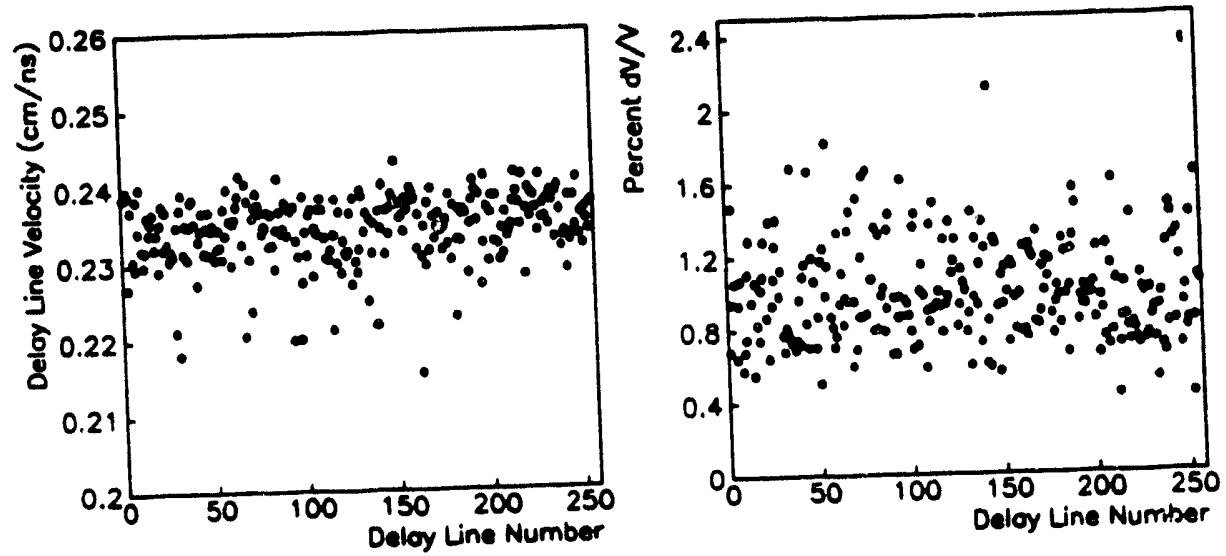

Figure 5.43: Fitted delay line mean velocities and percent $\mathrm{rms}$ deviation from the mean for all 256 delay lines.

is then determined from the requirement that equation 5.24 is satisfied

$$
\left.\frac{L}{2 V}\right|_{l}=\left\langle\frac{L}{V}\right\rangle-\left.\frac{L}{2 V}\right|_{r}=\frac{1}{16} \sum_{i=1}^{16}\left(T_{l i}+T_{r i}\right)-\left.\frac{L}{2 V}\right|_{r}
$$

Figure 5.43 shows the mean velocity as measured from the calibration procedure for all 256 delay lines. The average mean velocity is $.23435 \mathrm{~cm} / \mathrm{ns}$ with a line to line fluctuation of $2 \%$. Also shown is the percent rms velocity fluctuation within each delay line. The data suggests a uniform velocity to within $1 \pm .5 \%$ throughout the length of a typical line.

\subsection{Delay Line Resolution and Efficiency}

The delay line resolution and efficiency were measured by selecting a sample of events containing a track in both the $R \Phi$ and $R Z$ planes. The $R \Phi$ tracks were selected according to the constraints outlined in section 5.7. The $R Z$ 

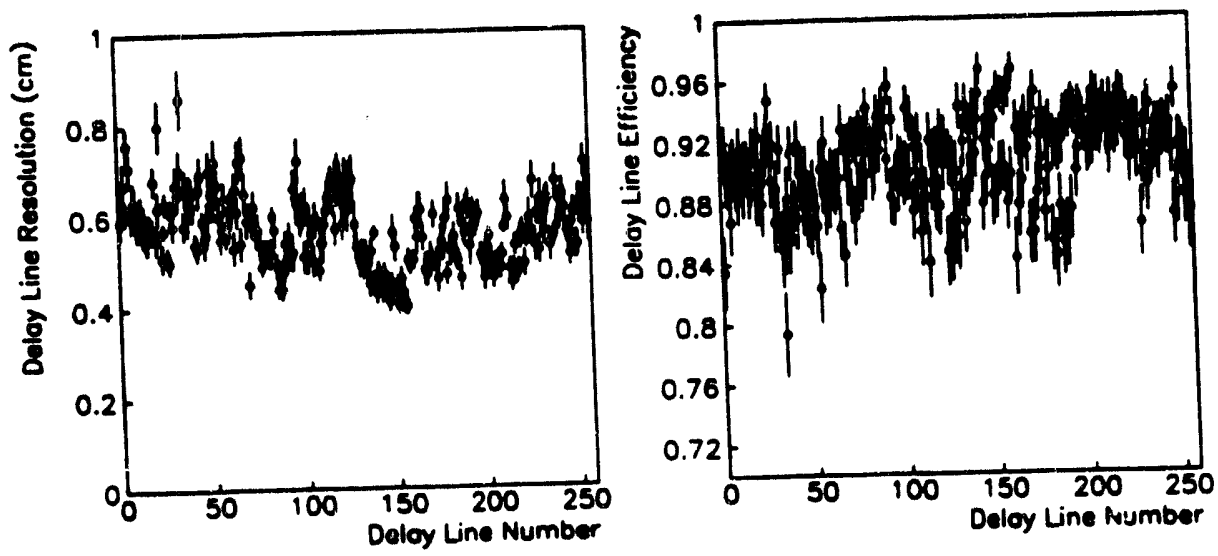

Figure 5.44: Delay line resolution with respect to a full $R Z$ track fit and efficiency. Results are for all 256 delay lines.

tracks were required to have a minimum of 4 out of 8 contributing delay line hits. Only delay lines which contained pulses on both ends and which satisfied $\left|Z_{l}-Z_{r}\right|<4 \mathrm{~cm}$ were accepted as part of an $R Z$ track.

Figure 5.44 shows the delay line resolution defined as the width of the residual distribution with respect to a fit of the full $R Z$ track. The results include corrections to compensate for the systematic biasing of the measured resolution when the particular line being studied is included in the fit. Also shown is the delay line efficiency defined as the probability that a delay line will yield a $Z$ coordinate satisfying the constraints of the previous paragraph when the driving sense wire hit is included in an $R \Phi$ track. The mean resolution for all lines is $.57 \mathrm{~cm}$ with a fluctuation of $.08 \mathrm{~cm}$. The mean efficiency is .91 with a line to line fluctuation of .03 . 


\section{Chapter 6}

\section{The Search For Top Quark Production at}

\section{Fermilab}




\subsection{Introduction}

The prospect of discovering the top quark in the upcoming collider run in March of 1992 at Fermilab and completing the quark generation structure of the standard model is eagerly anticipated. Previous searches for top by the $\mathrm{UA1}^{24}$ and $\mathrm{UA2}^{25}$ collaborations at the proton-antiproton collider at CERN have placed lower limits of 60 and $69 \mathrm{GeV}$ (95\% C.L.) respectively on the mass of the top $\left(M_{t}\right)$. Results from the Fermilab CDF experiment ${ }^{26}$ give a lower limit of $77 \mathrm{GeV}$ (95\% C.L.) while a recent preliminary CDF study ${ }^{27}$ suggests $M_{t}>89 \mathrm{GeV}$ (95\% C.L.). Theoretical upper limits on $M_{t}<210 \mathrm{GeV}$ and $M_{t}<200 \mathrm{GeV}$ have been given by Langacker ${ }^{28}$ and Barger ${ }^{29}$ from calculations based on radiative corrections to the $W$ and $Z$ masses. Since it seems likely that $M_{t}$ is within the range $90<M_{t}<200 \mathrm{GeV}$, the first chance of discovering top will be during upcoming collider runs at Fermilab.

\subsection{Top Production and Signal For $M_{t}>M_{W}$}

The dominant top quark production mechanism for $p \bar{p}$ collisions at Tevatron energies $(\sqrt{s}=1.8 \mathrm{TeV})$ is the QCD creation of $t \bar{t}$ pairs. Figure 6.1 shows the lowest order tree level Feynman graphs leading to a $q \bar{q}$ final state.

The production of $t \bar{t}$ pairs can be inferred through the identification of the decay products of the top quarks. According to the standard model, the top quark is expected to decay predominantly into its weak isospin partner the bottom quark and a $W$ boson. The $W$ will subsequently decay either into two quarks or into a lepton and neutrino (figure 6.2). For the case in which both $W$ 's decay leptonically, the final state topology consists of 2 jets from the $b$ quarks along with two high $p_{t}$ leptons and missing energy from the neutrinos. If only one $W$ decays leptonically, then one would expect 4 jets along with a single high $p_{t}$ lepton and missing energy. The case in which the 

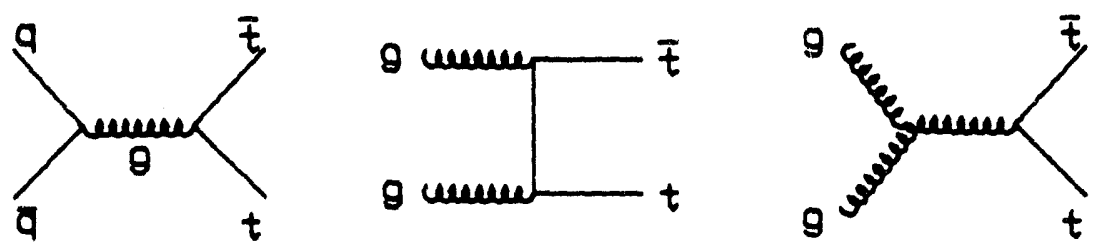

Figure 6.1: Feynman graphs contributing to the QCD creation of $t \bar{t}$.
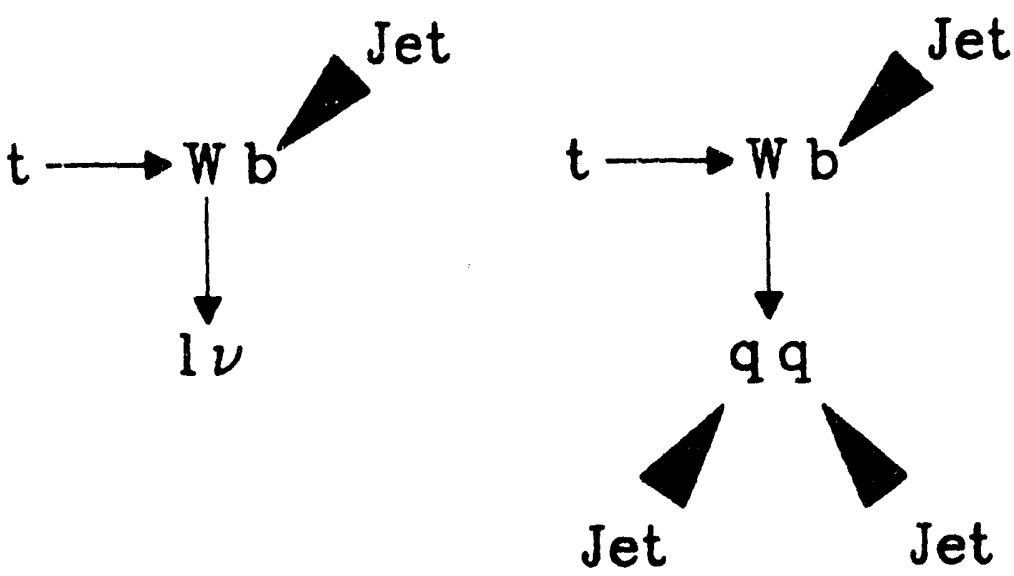

Figure 6.2: Decay modes of the top quark and the $W\left(M_{t}>M_{W}\right)$. 
$W$ decays into $\tau+\nu_{\tau}$ with the subsequent $\tau$ decay into either $e \nu_{e}$ or $\mu \nu_{\mu}$ can be distinguished from direct $W$ decay into $e$ or $\mu$ by the absence of a large $p_{t}$ lepton (most of the energy is carried away by the 2 neutrinos). The last case in which both $W$ 's decay into quarks would result in a 6 jet final state. The purely hadronic decay mode is difficult to observe due to the large QCD background. Note that if the mass of the top is near the current lower limit $\left(90<M_{t}<115 \mathrm{GeV}\right)$ it becomes unlikely that the low energy $b$ jet can be identified. A summary of the top signatures and their branching ratios (BR) is given in table 6.1 (excluding $W \rightarrow \tau \nu_{\tau}$ )

\begin{tabular}{||l|c||}
\hline \hline Top Signature & BR \\
\hline \hline$t \bar{t} \rightarrow b \bar{b}+4$ jets & .44 \\
$t \bar{t} \rightarrow b \bar{b}+2$ jets $+l_{\nu}$ & .30 \\
$t \bar{t} \rightarrow b \bar{b}+l_{1} \nu_{1}+l_{2} \nu_{2}$ & .05 \\
$l_{1} \neq l_{2}$ & .025 \\
$l_{1}=l_{2}$ & .025 \\
\hline \hline
\end{tabular}

Table 6.1 Top decay channels and branching ratios

\subsubsection{Top Search in Dilepton Channel}

The cleanest channel in which to search for the top signal is believed to be the dilepton channel in which the $W^{+} W^{-}$pair decay into different leptons $(e \mu)$. This decay mode has an advantage over the $e^{+} e^{-}$and $\mu^{+} \mu^{-}$final states due to the non existence of Drell-Yan and $Z^{\circ} \rightarrow l+l-$ backgrounds. The only backgrounds to the $e \mu$ channel are from $W$ pair production, $Z^{\circ} \rightarrow \tau^{+} \tau^{-}$, and heavy quark production and semileptonic decay. The $W W \rightarrow e \mu$ cross section is $\approx$ one third that of $t \bar{t} \rightarrow e \mu$ for $M_{t}=140 \mathrm{GeV}$. It is currently believed that a cut on the transverse momenta $>20 \mathrm{GeV}$ of the two highest 
jets gives $\approx$ a 15 to 1 rejection of the $W W$ background while preserving $\approx$ $95 \%$ of the top events. ${ }^{30}$

Heavy quark production $\left(Q C D \rightarrow b \bar{b}\right.$ and $\left.Z^{\circ} \rightarrow b \bar{b}\right)$ in which the $b$ quarks decay into $e \nu_{e}$ or $\mu \nu_{\mu}$ have been shown to be effectively eliminated ${ }^{27}$ by requiring

$$
\begin{aligned}
& E_{T}(e)>15 \mathrm{GeV} \\
& P_{T}(\mu)>15 \mathrm{GeV}
\end{aligned}
$$

It is believed that for $M_{T}$ as large as $140 \mathrm{GeV}$ and with the above lepton cuts, top signai to $b \bar{b}$ background ratio can be as large as 5 to $i$. Further Monte Carlo studies on the heavy flavor production backgrounds are currently underway. ${ }^{30}$

The remaining background to the $e \mu$ channel is from $Z^{0} \rightarrow \tau^{+} \tau^{-}$in which the $\tau$ s decay into $e \nu_{e}$ or $\mu \nu_{\mu}$. Such events were studied using the ISAJET V6.21 Monte Carlo. Table 6.2 $2^{31}$ shows the effect of various cuts on both the $t \bar{t}$ and $Z^{\circ} \rightarrow \tau^{+} \tau^{-}$rate for an integrated luminosity of $100 \mathrm{pb}^{-1}$ at center of mass energy $\sqrt{s}=1.8 \mathrm{TeV}$. The dilepton azimuthal seperation cut $(\Delta \Phi)$ in believed to be a good handle in effectively eliminating the $Z^{\circ}$ background, however, it must be noted that this is a preliminary result. Further studies are currently underway. In particular, a cut on the transverse mass $m_{T}\left(e \mu, P_{T}\right)^{32}$ defined as

$$
m_{T}^{2}\left(e \mu, P_{T}\right)=\left(\left(m_{e \mu}^{2}+P_{T e \mu}^{2}\right)^{1 / 2}+\left|P_{T}\right|\right)^{2}-\left(\mathbf{P}_{T e \mu}+\boldsymbol{P}_{T}\right)^{2}>M_{Z}^{2}
$$

where $P_{T}$ is the missing transverse momentum of the event and $M_{Z}$ is the $Z^{\circ}$ mass. The transverse mass for the $Z^{\circ} \rightarrow \tau^{+} \tau^{-}$background is bounded from above by the $Z^{\circ}$ mass. Preliminary investigations suggest that this cut is very effective in eliminating the $Z^{\circ}$ background, however, the top signal degradation could be as high as $50 \%$ for a top mass of $140 \mathrm{GeV} .^{30}$ 


\begin{tabular}{||l|c|c|c|c|c||}
\hline \multicolumn{5}{||c||}{ Number of Events $\left(100 b^{-1}\right.$ at $\left.\sqrt{s}=1.8 \mathrm{TeV}\right)$} \\
\hline \hline \multicolumn{1}{||c|}{ Cut } & $M_{t}=90$ & $M_{t}=120$ & $M_{t}=150$ & $M_{t}=180$ & $Z^{\circ} \rightarrow \tau^{+} \tau^{-}$ \\
\hline$E_{T}^{e}, P_{T}^{\mu}>15 \mathrm{GeV}$ & 326.6 & 76.6 & 22.0 & 7.8 & 124.6 \\
$|\eta|<1.2$ & 187.5 & 50.1 & 14.7 & 5.3 & 62.2 \\
$\Delta \Phi<150^{\circ}$ & 142.0 & 37.2 & 10.9 & 4.2 & 3.0 \\
\hline$E_{T}^{e}, P_{T}^{c_{1}}>20 \mathrm{GeV}$ & 272.1 & 65.4 & 19.0 & 6.8 & 51.0 \\
$|\eta|<1.2$ & 164.3 & 44.7 & 13.2 & 4.7 & 27.4 \\
$\Delta \Phi<150^{\circ}$ & 121.6 & 32.7 & 9.7 & 3.5 & 1.5 \\
\hline$E_{T}^{e}, P_{T}^{\mu}>30 \mathrm{GeV}$ & 155.8 & 39.1 & 12.0 & 4.5 & 4.8 \\
$|\eta|<1.2$ & 104.5 & 28.6 & 8.8 & 3.3 & 2.8 \\
$\Delta \Phi<150^{\circ}$ & 70.0 & 19.7 & 6.2 & 2.2 & 0.2 \\
\hline \hline
\end{tabular}

Table 6.2 $t \bar{t}$ and $Z^{\circ} \rightarrow \tau^{+} \tau^{-}$rate for various cuts.

\subsubsection{Top Search in Lepton + Jets Channel}

Although the branching ratio for $t \bar{t}$ into lepton + jets is 6 times greater than for the purely leptonic decay mode (table 6.1), one must contend with the serious background from $W+$ multijet production. The ability of seperating the top signal from background in events containing one high $P_{T}$ lepton +4 jets was studied using ISAJET for top masses of 120,150 , and $180 \mathrm{GeV} .{ }^{33}$ Only the channel in which one $W$ decays into $\mu \nu$ (the other into 2 jets) was considered in order to avoid the problem of isolating the electron from within jets. The events (and background) were passed through an energy smearing algorithm which simulates the expected resolution of the Do calorimeter. Jet finding was done using standard jet definitions. The jet selection criteria consisted of $\geq 4$ jets with $E_{T}>15 \mathrm{GeV}$ as these cuts appeared to minimally bias the top signal while effectively rejecting the $W+$ multijet background. 
The 4 momentum of the leptonically decaying $W$ was reconstructed from the measured muon 4 momentum $\left(P_{\mu}\right)$ and missing transverse momentum $P$ by constraining the invariant mass to be that of the $W$

$$
\left(P_{\mu}+P\right)^{2}=M_{W}^{2}
$$

This leads to a quadratic equation for the longitudinal missing momentum $P_{\mathbf{z}}$. It was found that the smaller solution gave the correct $P_{z} 85 \%$ of the time. A cut of 15 and $20 \mathrm{GeV}$ respectively was placed on the transverse momenta of the $\mu$ and reconstructed $W$.

The top signal was isolated by first requiring two jets within the event to have a mass within the $W$ band of 60 to $90 \mathrm{GeV}$. If such a pair was found, their energies were scaled so as to force a dijet mass of $81 \mathrm{GeV}$. One of the remaining jets was then paired with the leptonically reconstructed $W$ and the invariant mass of the $W+$ jet combination $m_{1}$ was formed. The remaining jets were then cycled through and combined with with the dijet pair to give an invariant mass $m_{2}$. The jet combination which gave the closest match between $m_{1}$ and $m_{2}$ was chosen.

Figure 6.3 shows the invariant mass distributions of the $W$-jet pair after minimizing the $m_{1}, m_{2}$ difference for top masses of 120,150 , and $180 \mathrm{GeV}$. Additional jet cuts applied were on the largest and second largest jet in the event and were $45,50,55 \mathrm{GeV}$ and $30,35,40 \mathrm{GeV}$ respectively for top masses of 120,150 , and $180 \mathrm{GeV}$. It is interesting to note that although the correct $b$ jet is associated with the $W$ only $35 \%$ of the time, there still emerges a peak at the correct top mass for all 3 cases considered. It seems, therefore, that tagging the $b$ quark via the semileptonic decay $b \rightarrow c \mu \nu$ is probably not necessary, especially since it would substantially reduce the available top signal.

An investigation of the $W+$ multijet background showed that the signal to background in the $\mu \nu+$ jets channel could be as high as 20 to 1 for a 
top mass of $120 \mathrm{GeV}$ and 10 to 1 for a top mass of $180 \mathrm{GeV}$. In addition, a refined analysis of the $e+$ jets channel is currently underway in which a sample of signal and background events were filtered through the complete DO detector simulation program (GEANT). Very preliminary results including an optimized event selection criteria suggests that the signal to background in this channel could be as low as 2 to 1 for a top mass of $120 \mathrm{GeV} .^{34}$

\subsection{Conclusion}

The backgrounds to the top signal in both the dilepton and lepton + jets channels are believed to be controllable with appropriate cuts on the lepton and jet energies. With an estimated $20 \mathrm{pb}^{-1}$ of data expected in the upcoming Fermilab collider run in March of 1992, the maximum top mass reach is 150 to $160 \mathrm{GeV}$. A Monte Carlo study including the full detector simulation (GEANT) suggests that for a top mass of $150 \mathrm{GeV}$, one would expect $\approx 2$ to $3 \mathrm{e} \mu$ events, $\approx 1$ ee or $\mu \mu$ event, and $\approx 15$ events for both $e+$ jets and $\mu+$ jets. It is important to see all the signatures in the correct proportion before discovery of the top quark can be claimed. 

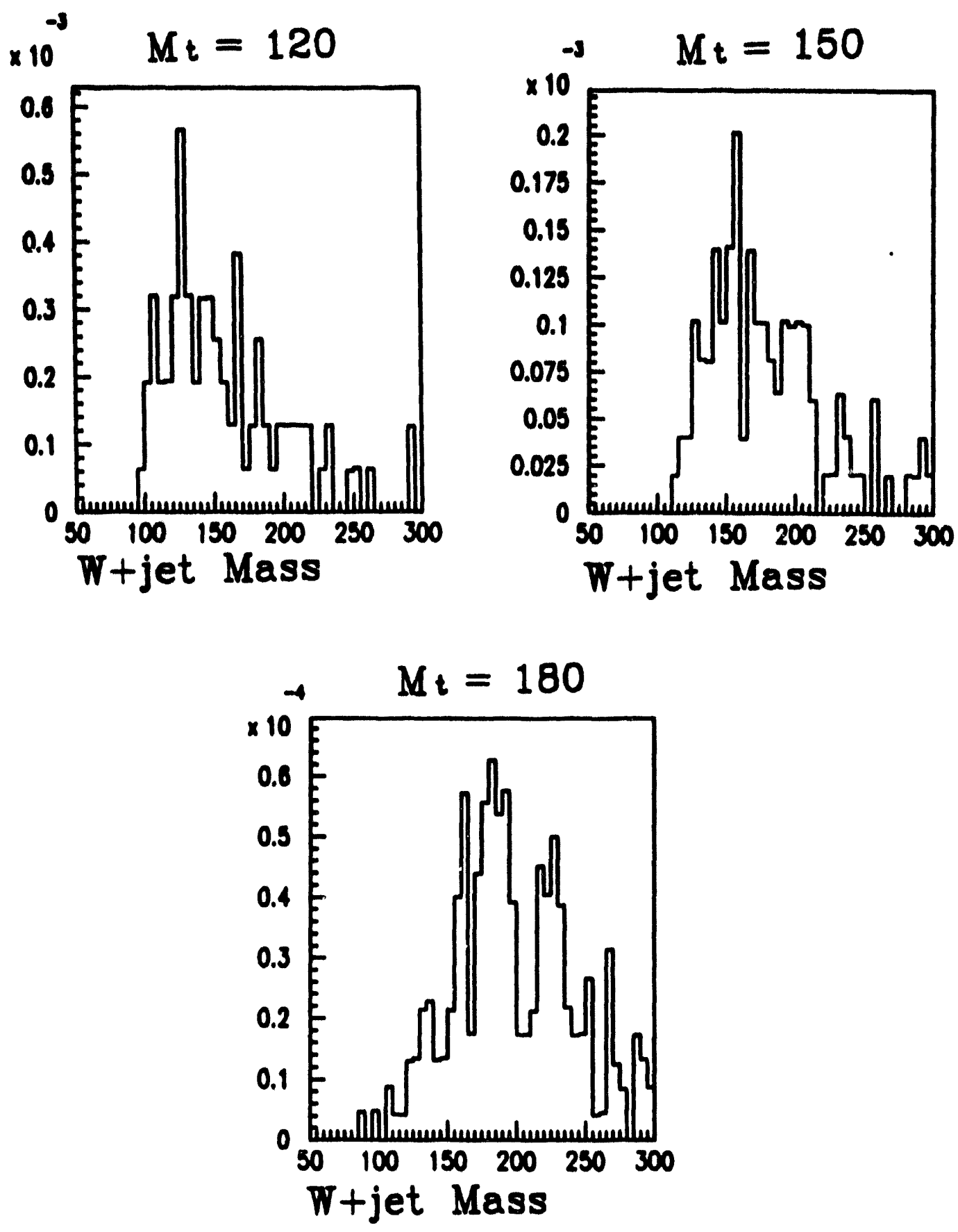

Figure 6.3: Invariant mass distributions of best $W$-jet pair for 3 different top quark masses. 


\section{Bibliography}

[1] Fisk H. E. and Slaughter J. Collider Physics at Fermilab in the 1990's. 1990. D0 Note 934.

[2] Barger V. D. and Phillips R. J. N. Collider Physics. Addison Wesley, 1962.

[3] Oltman E. et. al. Do Vertex Drift Chamber Construction and Test Results. 1991. Do Note 1169.

[4] Jackson J. D. Classical Electrodynamics. Wiley, 1962.

[5] Detoeuf J. et. al. The Do Transition Radiation Detector. 1987. D0 Note 528.

[6] Grannis P. The Do Detector at the Fermilab Collider. 1990. Do Note 558.

[7] Grannis P. Specifications for the Do Detector. 1990. D0 Note 560.

[8] Do Collaboration. The DO Experiment at the Fermilab AntiprotonProton Collider. 1984. Design Report.

[9] Franzini P. Performance of the Do Uranium-Liquid Argon Calorimeter Modules. Nucl. Instr. and Meth., A288:438, (1990).

[10] Womersley W. J. Hadron Position Resolution in ECMH. 1988. D0 Note 785. 
[11] Behnke T. The Central Drift Chamber for the Do Experiment: Design, Construction and Test. PhD thesis, State University of New York at Stony Brook, 1989.

[12] Rijssenbeek M. et. al. 8 Channel Preamp Hybrid Based on the Fujitsu MB43458 Quad Charge Amplifier. 1987. DO Note 563.

[13] Saewert G. et. al. Central Tracking Detector Flash $A / D$ Converter Buffer Circuit Summary. 1988. Do Note 669.

[14] Abolins M. The Level one Framework. 1988. D0 Note 705.

[15] Utes M. DO Central Tracking FADC Controller Module. 1990. D0 Note 962.

[16] Linnemann J. The Level one Framework. 1991. D0 Note 1230.

[17] Lorentz H. A. Theory of Electrons. Dover, 1952.

[18] Morse P.M., Allis W.P. and Lamar E.S. Velocity Distributions for Elastically Colliding Electrons. Phys. Rev., 48:412, (1935).

[19] Huxley L.G.H. and Crompton R. W. The Diffusion and Drift of Electrons in Gases. Wiley, 1974.

[20] Schultz G. and Gresser J. A Study of Transport Coefficients of Electrons in Some Gases Used in Proportional and Drift Chambers. Nucl. Instr. and Meth., 151:413, (1978).

[21] Ferbel T. Experimental Techniques in High Energy Physics. AddisonWesley, 1987. See article by Sauli Principles of Operation of Multiwire Proportional and Drift Chambers.

[22] Rajagopolan S. private communication. 
[23] Bilger R. Measuring Drift Velocities with a Drift Chamber. 1988. Internal Report.

[24] Albajar C. et al. Submitted to Z. Phys.

[25] Akesson T. et al. (1990).

[26] Abe F. et. al. Search for the Top Quark in the Reaction $p \bar{p} \rightarrow$ Electron + Jets at $\sqrt{s}=1.8$ Tev. Phys. Rev. Lett., 64:142, (1990).

[27] Campagnari C. et al. Top Physics at CDF.

[28] Langacker P. Implications of Recent $M_{Z, W}$ and Neutral Current Measuzements for the Top Quark Mass. Phys. Rev. Lett., 63:1920, (1989).

[29] Barger V. Top Quark Mass Predictions From $W, Z$ Masses and $Z$ Partial Widths. Phys. Rev. Lett., 65:1313, (1990).

[30] Cochran J. private communication.

[31] Abachi S. et. al. Prospects for Top at the Tevatron Collider in the 1990's. 1989.

[32] Baer H. et. al. Detecting Very Massive Top Quarks at the Fermilab Tevatron. Phys. Rev. D, 42:54, (1990).

[33] Grannis P. Isolating Heavy Top in Lepton + Jets. 1990. D0 Note 928.

[34] Chakraborty D. private communication. 

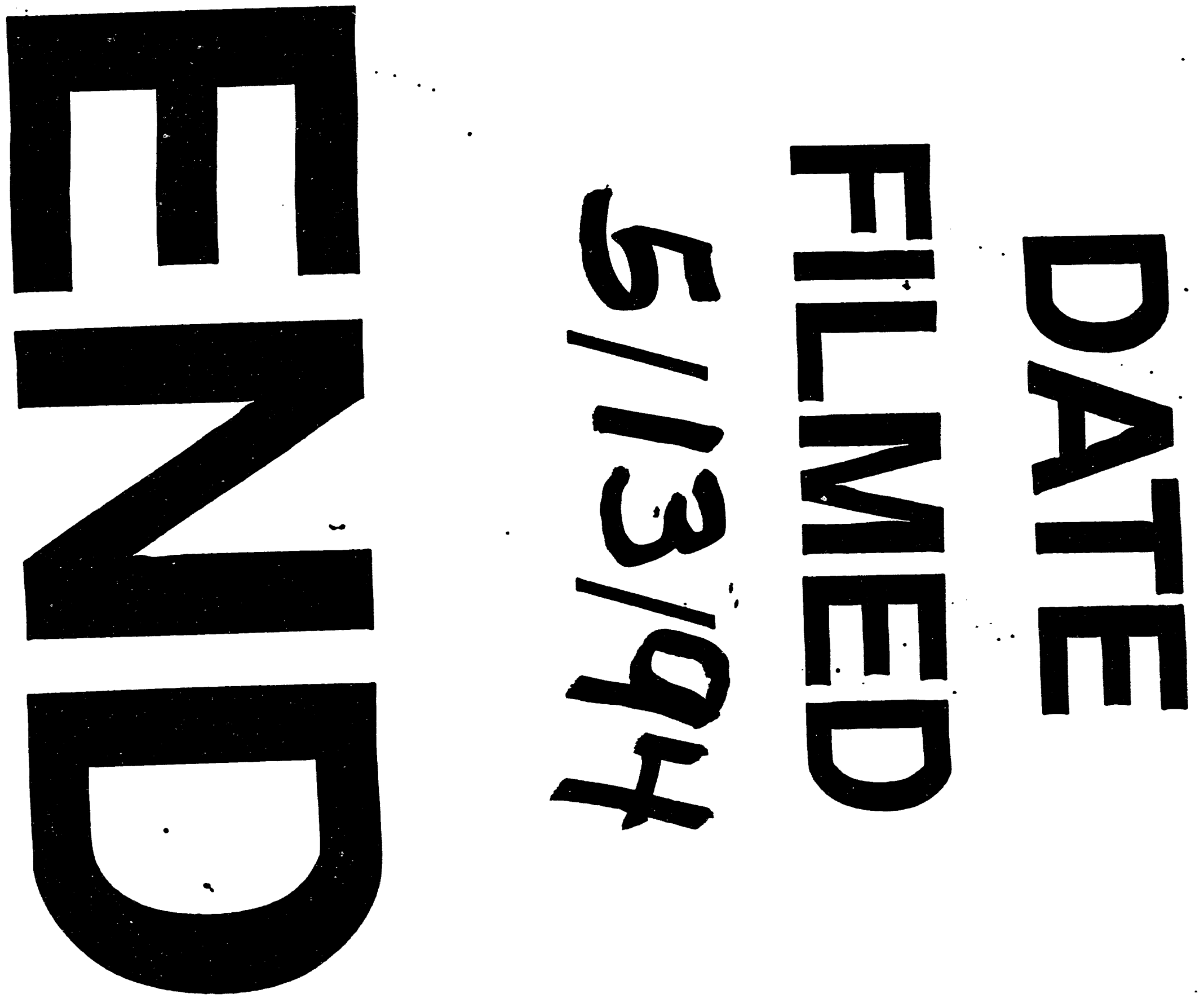